\title{
Generalized spectral categories, topological Hochschild homology and trace maps
}

\author{
GONÇALO TABUADA
}

\begin{abstract}
Given a monoidal model category $\mathcal{C}$ and an object $K$ in $\mathcal{C}$, Hovey constructed in [22] the monoidal model category $\mathrm{Sp}^{\Sigma}(\mathcal{C}, K)$ of $K$-symmetric spectra over $\mathcal{C}$. In this paper we describe how to lift a model structure on the category of $\mathcal{C}$-enriched categories to the category of $\mathrm{Sp}^{\Sigma}(\mathcal{C}, K)$-enriched categories. This allow us to construct a (four step) zig-zag of Quillen equivalences comparing dg categories to $H \mathbb{Z}$-categories. As an application we obtain: (1) the invariance under weak equivalences of the topological Hochschild homology $(T H H)$ and topological cyclic homology $(T C)$ of dg categories; (2) non-trivial natural transformations from algebraic $K$-theory to THH.
\end{abstract}

55P42, 18D20, 18G55; 19D55

\section{Introduction}

Given a symmetric monoidal model category $\left(\mathcal{C},-\otimes_{\mathcal{C}}-, \mathbf{1}_{\mathcal{C}}\right)$, one can consider the category $\mathcal{C}-$ Cat of all small categories enriched over $\mathcal{C}$ with varying object set, see Notation 2.2. When $\mathcal{C}$ is the category of simplicial sets these are called simplicial categories (see Dwyer and Kan $[12 ; 13]$ ), when $\mathcal{C}$ is the category of chain complexes these are called differential graded $(=\mathrm{dg}$ ) categories (see Drinfeld [6] and Keller [25]) and when $\mathcal{C}$ is the category of symmetric spectra (see Hovey, Shipley and Smith [23]) these are called spectral categories (see Blumberg and Mandell [2] and Schwede and Shipley [31]). If we denote by $[-,-]$ the set of morphisms in the homotopy category $\mathrm{Ho}(\mathcal{C})$ of $\mathcal{C}$, we have a natural functor

$$
[-]: \mathcal{C}-\mathrm{Cat} \longrightarrow \text { Cat }
$$

obtained by base change along the composed functor

$$
\mathcal{C} \longrightarrow \mathrm{Ho}(\mathcal{C}) \stackrel{\left[1_{\mathcal{C}},-\right]}{\longrightarrow} \text { Set } .
$$

The novelty of $\mathcal{C}-$ Cat is that it is endowed with a natural notion of weak equivalence which mixes the weak equivalences in $\mathcal{C}$ with categorical equivalences. 
Definition 1.1 A $\mathcal{C}$-functor $F: \mathcal{A} \rightarrow \mathcal{B}$ is called a weak equivalence if

(WE1) for all objects $x, y \in \mathcal{A}$, the morphism

$$
F(x, y): \mathcal{A}(x, y) \longrightarrow \mathcal{B}(F x, F y)
$$

is a weak equivalence in $\mathcal{C}$ and

(WE2) the induced functor

$$
[F]:[\mathcal{A}] \longrightarrow[\mathcal{B}]
$$

is an equivalence of categories.

In simplicial, differential graded and spectral categories this notion reduces to the classical notions of Dwyer-Kan equivalence (see Dwyer and Kan [12, Section 2.4]), quasi-equivalence (see Drinfeld [6, Section 2.3] and Keller [25, Section 2.3]) and Dwyer-Kan equivalence (see Blumberg and Mandell [2, Definition 4.1]) or equivalently stable quasi-equivalence (see Tabuada [37, Definition 5.1]). In what concerns spectral categories, we would like to mention Dugger's work [8]. He proved that every stable, presentable model category $\mathcal{M}$ can be enriched over symmetric spectra, thus giving rise to a spectral category. In order to compare all the possible spectral enrichments of $\mathcal{M}$, Dugger introduced a notion of weak equivalence defined in terms of bimodules (see [8, Section 3]) and showed that up to this notion the enrichment of $\mathcal{M}$ is canonical. Let us now focus on Quillen model structures.

- in the case of $\mathcal{C}$-enriched categories with a single object (for example, simplicial monoids, dg algebras, or symmetric ring spectra) or more generally $\mathcal{C}$-enriched categories with a fixed set of objects (see Notation 2.2), Schwede and Shipley developed a very general theory for constructing model structures, see [30, Section 6].

- in the case of $\mathcal{C}$-enriched categories with varying object set, a general theory of model structures (with weak equivalences given by Definition 1.1) has not yet been developed. The reason for this is condition (WE2), which makes all the arguments very sensitive to the choice of the base monoidal category $\mathcal{C}$. Nevertheless, model structures were built on the specific contexts of simplicial categories (see Bergner [1]), dg categories (see Tabuada [34]) and spectral categories (see Tabuada [37]).

The main goal of this paper is the development of a general theory of model structures on generalized spectral categories.

Generalized spectral categories Hovey developed in [22] a homotopy theory of symmetric spectra in general Quillen model categories. Starting from a "well-behaved" 
monoidal model category $\mathcal{C}$ and a cofibrant object $K$ in $\mathcal{C}$, Hovey constructed the stable monoidal model category $\operatorname{Sp}^{\Sigma}(\mathcal{C}, K)$ of $K$-symmetric spectra over $\mathcal{C}$. When $\mathcal{C}$ is the category of pointed simplicial sets, Hovey's construction reduces to the classical construction of symmetric spectra (see Hovey, Shipley and Smith [23]).

In this paper we describe how to lift a Quillen model structure on $\mathcal{C}-$ Cat to the category $\mathrm{Sp}^{\Sigma}(\mathcal{C}, K)-$ Cat of generalized spectral categories. Our main Theorem is the following.

\section{Theorem 1.2 (see Theorem 3.46) Given}

- a symmetric monoidal model category $\mathcal{C}$ and a cofibrant object $K$ in $\mathcal{C}$ (satisfying the conditions of Section 3.1) and

- a Quillen model structure on $\mathcal{C}-$ Cat (satisfying the conditions of Section 3.2),

there exists an induced Quillen model structure on $\operatorname{Sp}^{\Sigma}(\mathcal{C}, K)-C a t$, with weak equivalences given by Definition 1.1.

The conditions of our main Theorem are very general and in Examples 3.1 and 3.10 we show that they are verified in all the familiar examples (that is, when $\mathcal{C}$ is the category of: pointed simplicial sets $s$ Set., chain complexes $\mathbf{C h}$, positively graded chain complexes $\mathbf{C h}_{\geq 0}$ and simplicial abelian groups $s \mathbf{A b}$ ). The construction of the model structure on $\mathrm{Sp}^{\Sigma}(\mathcal{C}, K)-$ Cat, which is inspired by the one on spectral categories (see Tabuada [37, Theorem 5.10]) is performed in two steps: first, we lift the model structure on $\mathcal{C}$-Cat to $\operatorname{Sp}^{\Sigma}(\mathcal{C}, K)$-Cat along a natural adjunction (see Theorem 3.19) and obtain a model structure whose weak equivalences are the levelwise quasi-equivalences, see Definition 3.14. Then, using the generating trivial cofibrations of the stable model structure on $\mathrm{Sp}^{\Sigma}(\mathcal{C}, K)$, we construct a functor (see Section 3.9)

$$
Q: \mathrm{Sp}^{\Sigma}(\mathcal{C}, K)-\mathrm{Cat} \longrightarrow \mathrm{Sp}^{\Sigma}(\mathcal{C}, K)-\mathrm{Cat},
$$

which we use to localize the levelwise model structure and obtain the desired model structure on $\operatorname{Sp}^{\Sigma}(\mathcal{C}, K)-$ Cat.

Now, let $R$ be a commutative symmetric ring spectrum (for example, the EilenbergMac Lane symmetric ring spectrum $H \mathbb{Z}$ of $\mathbb{Z}$ ). Using arguments conceptually very similar to those of Theorem 3.46, we construct in Theorem 5.17 a Quillen model structure on the category $R$-Cat of small categories enriched over $R$-modules.

Theorems 3.46 and 5.17 combined with a homotopy idempotence result (see Theorem 3.52), a general Quillen equivalence criterion (see Theorem 4.2 and Section 4.1), 
Proposition 6.1, and Shipley's zig-zag machinery [32], furnishes us a (four step) zigzag of Quillen equivalences (see Section 7)

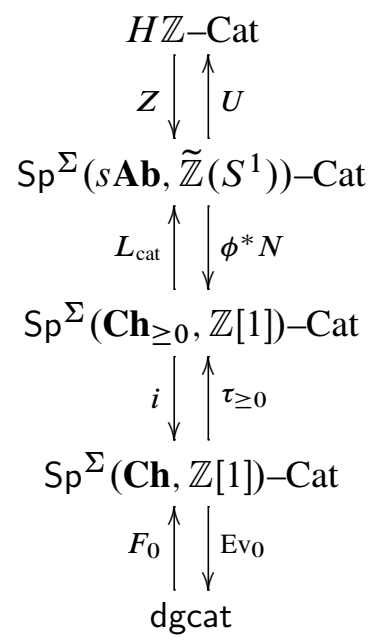

comparing dg categories to $H \mathbb{Z}$-categories (see Notation 5.3). Notice that in each step the weak equivalences are always those of Definition 1.1 and when we restrict to enriched categories with a single object we recover Shipley's zig-zag [32], comparing dg algebras to $H \mathbb{Z}$-algebras. Let us now describe two applications of this zig-zag.

Topological Hochschild homology In the past two decades, topological Hochschild homology $(T H H)$ and topological cyclic homology $(T C)$ have revolutionized algebraic $K$-theory computations, see for instance Hesselholt and Madsen [17; 18; 19]. Roughly, the $T H H$ of a ring is obtained by promoting the ring to a symmetric ring spectrum and substituting the tensor product in the classical Hochschild complex by the smash product. The THH spectrum comes with a "cyclotomic" structure, and for each prime $p, T C$ is defined as a certain homotopy limit over the fixed point spectra.

Using Thomason's hypercohomology construction [39], Geisser and Hesselholt introduced in a pioneer work [14] the topological Hochschild homology and topological cyclic homology of schemes, and performed several important computations, see for instance [14, Section 5.3].

More recently, Blumberg and Mandell introduced in [2] (see Definition 8.1) the topological Hochschild homology and topological cyclic homology of dg categories. Their construction can be decomposed in two steps:

(1) Building upon the work of McCarthy and Dundas [11] (which, in turn, was based on Bökstedt's original construction [3]) they defined $T H H$ and $T C$ for spectral categories. 
(2) Then, by applying Shipley's machinery [32], they constructed a zig-zag of Quillen equivalences comparing dg categories to $H \mathbb{Z}$-categories, analogous to the one of the previous Subsection but where the weak equivalences are the enriched functors which induce a bijection on the object sets and satisfy condition (WE1). Using the natural restriction functor from $H \mathbb{Z}$-categories to spectral ones, they obtained a functor from dg categories to spectral categories.

Given a dg category $\mathcal{A}$, its $T H H$ and $T C$ is by definition the pre-composition of the functor of item (2) with the functors $T H H$ and $T C$ (for spectral categories). In conclusion, Blumberg and Mandell obtained well-defined functors

$$
T H H, T C: \text { dgcat } \longrightarrow \mathrm{Sp}^{\Sigma} \text {. }
$$

Since the comparison between dg categories and spectral ones, described in item (2), makes use of model structures which don't have the correct weak equivalences (since these weak equivalences induce a bijection on the object sets), it is not clear a priori if $T H H$ and $T C$ are invariant under weak equivalences. Our zig-zag of Quillen equivalences furnishes a conceptual proof of this fact.

Proposition 1.3 (see Proposition 8.3) The functors $T H H$ and TC, from dg categories to symmetric spectra, send the weak equivalences of Definition 1.1 to stable equivalences.

Given a scheme $X$, Blumberg and Mandell defined its $T H H$ and $T C$ by applying

$$
T H H, T C: \text { dgcat } \longrightarrow \mathrm{Sp}^{\Sigma}
$$

to a dg category $\mathcal{D}_{\text {perf }}^{\mathrm{DG}}(X)$ modeling the derived category of perfect complexes of $\mathcal{O}_{X}$-modules. They proved in [2, Theorem 1.3] that when $X$ is quasi-compact and semi-separated their definition coincides with the one of Geisser-Hesselholt.

Thanks to Proposition 1.3, we now have the "flexibility" of choosing for $\mathcal{D}_{\text {perf }}^{\mathrm{DG}}(X)$ any $\mathrm{dg}$ category which models the derived category of perfect complexes of $\mathcal{O}_{X}$-modules. For example, we can take for $\mathcal{D}_{\text {perf }}^{\mathrm{DG}}(X)$ the dg category of bounded complexes of injective $\mathcal{O}_{X}$-modules with coherent homology.

Trace maps Given a scheme $X$, Geisser and Hesselholt constructed in [14] the cyclotomic trace map from the algebraic $K$-theory of $X$ to its topological Hochschild homology and topological cyclic homology. This cyclotomic trace map was one of the main working tools which allowed them to make extensive $K$-theoretic computations, see for instance [14, Section 5]. Recall from the previous Subsection that when $X$ is quasi-compact and semi-separated, Blumberg-Mandell's definition coincides with 
the one of Geisser-Hesselholt. This implies that there are well-defined cyclotomic trace maps from the algebraic $K$-theory of $X$ to $\operatorname{THH}\left(\mathcal{D}_{\text {perf }}^{\mathrm{DG}}(X)\right)$ and $T C\left(\mathcal{D}_{\text {perf }}^{\mathrm{DG}}(X)\right)$. Blumberg and Mandell improved this by giving in [2, Appendix B] an intrinsic construction of the cyclotomic trace for certain dg categories like $\mathcal{D}_{\text {perf }}^{\mathrm{DG}}(X)$, which come from complicial Waldhausen ones.

Using Proposition 1.3 and a result concerning the co-representability of algebraic $K$-theory (see Tabuada [36, Theorem 15.10]), we construct natural transformations from algebraic $K$-theory to topological Hochschild homology.

\section{Proposition 1.4 (see Proposition 8.10) Let}

$$
K(-), T H H(-): \mathrm{HO}(\text { dgcat }) \longrightarrow \mathrm{HO}\left(\mathrm{Sp}^{\Sigma}\right)
$$

be the morphisms of derivators naturally associated to algebraic $K$-theory and topological Hochschild homology. We have non-trivial natural transformations of morphisms of derivators

$$
\begin{aligned}
& \text { (i): } K(-) \Rightarrow T H H(-) \\
& \text { (ii): } K(-) \Rightarrow T H H[-(2 r-1)](-), \quad r \geq 1
\end{aligned}
$$

where THH[-(2r-1)](-) denotes the composition of THH $(-)$ with the $(-(2 r-1))-$ suspension functor in $\mathrm{HO}\left(\mathrm{Sp}^{\Sigma}\right)$. By applying the functors

$$
\pi_{n}^{s}: \mathrm{Ho}\left(\mathrm{Sp}^{\Sigma}\right) \longrightarrow \operatorname{Mod}-\mathbb{Z}, n \geq 0
$$

to the previous natural transformations, we obtain non-trivial natural transformations of functors

$$
\begin{aligned}
& \text { (i): } K_{n}(-) \Rightarrow T H H_{n}(-), \quad n \geq 0 \\
& \text { (ii): } K_{n}(-) \Rightarrow T H H_{n+2 r-1}(-), \quad n \geq 0, r \geq 1 .
\end{aligned}
$$

The natural transformations of Proposition 1.4 have the advantage of being available for all dg categories, but the disadvantage of being defined only over $\mathbb{Z}$ (or over $\mathbb{Z} / p \mathbb{Z}$ with $p$ prime, see Proposition 8.12). At the present time the author does not know how to relate these natural transformations with the cyclotomic trace. The reason for this is that, in contrast with Geisser-Hesselholt and Blumberg-Mandell's work, our construction is given in an implicit way (making use of a co-representability result, see Tabuada [36, Theorem 15.10]). However, taking into account the naturality of all these constructions, it is expected that in the particular case of a quasi-compact and separated scheme $X$ they all coincide. This will be the subject of future research.

In Section 9, we recall Dugger-Shipley's notion of topological equivalence [9] and show that over $\mathbb{Q}$, two dg categories are topologically equivalent if and only if they are quasi-equivalent, see Proposition 9.5. We have included four appendices. In Appendix 
A we construct a general adjunction, which is used throughout the paper. In Appendix $\mathrm{B}$ we recall and generalize some results concerning the construction and localization of Quillen model structures. In Appendix $C$ we give an explicit description of certain pushouts used in the construction of our model structures. Finally, in Appendix D we prove all the results stated in Section 5.

\subsection{Acknowledgments}

It is a great pleasure to thank Stefan Schwede for suggesting me this problem and Gustavo Granja for helpful conversations. I am deeply grateful to the anonymous referee for his highly detailed comments which allowed me to greatly improve this paper. Finally, I would like also to thank the Institute for Advanced Study at Princeton for excellent working conditions, where part of this work was carried out. The author was partially supported by the grant SFRH/BPD/34575/2007 FCT-Portugal.

\section{Preliminaries}

Throughout the paper we will be working (to simplify notation) over the ring of integers $\mathbb{Z}$. However, all our results stay valid if we replace $\mathbb{Z}$ by any other commutative ring. We will use freely the language of homotopical algebra. The reader unfamiliar with this language is invited to consult Hirschhorn [20], Hovey [21], Goerss and Jardine [15] or Quillen's original monograph [27].

Notation 2.1 Let $\mathbf{C h}$ denote the category of complexes and $\mathbf{C h} \geq 0$ the full subcategory of positively graded complexes, see Hovey [21, Section 2.3]. We consider homological notation, that is, the differential decreases the degree. We denote by $s$ Set, resp. by $s$ Set., the category of simplicial sets, resp. pointed simplicial sets, (see Goerss and Jardine [15, Chapter I]) and by $s \mathbf{A b}$ be the category of simplicial abelian groups, see [15, Section III-2]. Finally, let $\mathrm{Sp}^{\Sigma}$ be the category of symmetric spectra of pointed simplicial sets, see Hovey, Shipley and Smith [23] or Schwede [28].

Notation 2.2 Let $\left(\mathcal{C},-\otimes_{\mathcal{C}}-, \mathbf{1}_{\mathcal{C}}\right)$ be a symmetric monoidal category, see Borceaux [4, Definition 6.1.2]. We denote by $\mathcal{C}-$ Cat the category of small categories enriched over $\mathcal{C}$, see [4, Definition 6.2.1]. An object in $\mathcal{C}$-Cat will be called a $\mathcal{C}$-category and a morphism a $\mathcal{C}$-functor. Notice that the category $\mathcal{C}$-Cat consists of all small categories enriched over $\mathcal{C}$ with varying object set. If $I$ is a set, we denote by $\mathcal{C}^{I}$-Cat the category of small categories enriched over $\mathcal{C}$ which have a fixed set of objects $I$, see Schwede and Shipley [30, Section 6]. The morphisms in $\mathcal{C}^{I}$-Cat induce the identity map on the object sets. 
Notation 2.3 Let $\mathcal{D}$ be a Quillen model category. We denote by $\mathcal{W}_{\mathcal{D}}$ its class of weak equivalences and by $[-,-]_{\mathcal{D}}$ the set of morphisms in the homotopy category $\mathrm{Ho}(\mathcal{D})$ of $\mathcal{D}$. Moreover, if $\mathcal{D}$ is cofibrantly generated, we denote by $I_{\mathcal{D}}$, resp. by $J_{\mathcal{D}}$, its set of generating cofibrations, resp. generating trivial cofibrations.

Definition 2.4 (see Mac Lane [26, Section XI-2]) Let $\left(\mathcal{C},-\otimes-, \mathbf{1}_{\mathcal{C}}\right)$ and $(\mathcal{D},-\wedge$ ,$\left.- \mathbf{1}_{\mathcal{D}}\right)$ be two symmetric monoidal categories. A lax monoidal functor is a functor $F: \mathcal{C} \rightarrow \mathcal{D}$ equipped with:

- a morphism $\eta: \mathbf{1}_{\mathcal{D}} \rightarrow F\left(\mathbf{1}_{\mathcal{C}}\right)$ and

- natural morphisms

$$
\psi_{X, Y}: F(X) \wedge F(Y) \rightarrow F(X \otimes Y), \quad X, Y \in \mathcal{C}
$$

which are coherently associative and unital.

A lax monoidal functor is strong monoidal if the morphisms $\eta$ and $\psi_{X, Y}$ are isomorphisms.

Suppose we have an adjunction

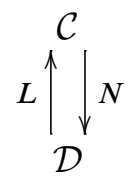

with $N$ a lax monoidal functor. Recall from Schwede and Shipley [30, Section 3.2] that the left adjoint $L$ is endowed with a lax comonoidal structure.

$$
\begin{array}{cccc}
\gamma: & L\left(\mathbf{1}_{\mathcal{D}}\right) & \longrightarrow & \mathbf{1}_{\mathcal{C}} \\
\phi: & L(X \wedge Y) & \longrightarrow & L(X) \otimes L(Y) .
\end{array}
$$

Definition 2.5 (see Schwede and Shipley [30, Definition 3.6]) A weak monoidal Quillen pair

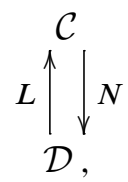

between monoidal model categories $\mathcal{C}$ and $\mathcal{D}$ consists of a Quillen pair, with a lax monoidal structure on the right adjoint, such that the following two conditions hold:

(i) for all cofibrant objects $X$ and $Y$ in $\mathcal{D}$, the comonoidal map

$$
\phi: L(X \wedge Y) \longrightarrow L(X) \otimes L(Y)
$$

belongs to $\mathcal{W}_{\mathcal{C}}$ and 
(ii) for some (hence any) cofibrant resolution $\mathbf{1}_{\mathcal{D}}^{c} \stackrel{\sim}{\rightarrow} \mathbf{1}_{\mathcal{D}}$, the composite map

$$
L\left(\mathbf{1}_{\mathcal{D}}^{c}\right) \rightarrow L\left(\mathbf{1}_{\mathcal{D}}\right) \stackrel{\gamma}{\rightarrow} \mathbf{1}_{\mathcal{C}}
$$

belongs to $\mathcal{W}_{\mathcal{C}}$.

A strong monoidal Quillen pair is a weak monoidal Quillen pair for which the comonoidal maps $\gamma$ and $\phi$ are isomorphisms. A weak (resp. strong) monoidal Quillen pair is a weak monoidal Quillen equivalence (respectively a strong monoidal Quillen equivalence) if the underlying pair is a Quillen equivalence.

Remark 2.6 If $\mathbf{1}_{\mathcal{D}}$ is cofibrant and $L$ is strong monoidal, then $N$ is lax monoidal and the Quillen pair is a strong monoidal Quillen pair.

Let $\mathcal{C}$ a symmetric monoidal model category, see Hovey [21, Definition 4.2.6].

Remark 2.7 We have a functor

$$
[-]: \mathcal{C}-\text { Cat } \longrightarrow \text { Cat },
$$

with values in the category of small categories, obtained by base change along the composed functor

$$
\mathcal{C} \longrightarrow \mathrm{Ho}(\mathcal{C}) \stackrel{\left[1_{\mathcal{C}},-\right]}{\longrightarrow} \text { Set } .
$$

Definition 2.8 A $\mathcal{C}$-functor $F: \mathcal{A} \rightarrow \mathcal{B}$ is a weak equivalence if:

(WE1) for all objects $x, y \in \mathcal{A}$, the morphism

$$
F(x, y): \mathcal{A}(x, y) \longrightarrow \mathcal{B}(F x, F y)
$$

belongs to $\mathcal{W}_{\mathcal{C}}$ and

(WE2) the induced functor

$$
[F]:[\mathcal{A}] \longrightarrow[\mathcal{B}]
$$

is an equivalence of categories.

Remark 2.9 If condition (WE1) is verified, then condition (WE2) is equivalent to:

(WE2') the induced functor

$$
[F]:[\mathcal{A}] \longrightarrow[\mathcal{B}]
$$

is essentially surjective. 


\section{Example 2.10}

- When $\mathcal{C}$ is the category of simplicial sets (see Goerss and Jardine [15, Theorem 11.3-I]), the notion of weak equivalence corresponds to the notion of Dwyer-Kan equivalence (see Dwyer and Kan [12, Section 2.4]).

- When $\mathcal{C}$ is the category of complexes (see Hovey [21, Theorem 2.3.11]), the notion of weak equivalence corresponds to the notion of quasi-equivalence (see Drinfeld [6, Section 2.3] and Keller [25, Section 2.3]).

- When $\mathcal{C}$ is the category of symmetric spectra (see Schwede [28, Theorem 2.2(i)III]), the notion of weak equivalence corresponds to the notion of Dwyer-Kan equivalence (see Blumberg and Mandell [2, Definition 4.1]) or equivalently to the notion of stable quasi-equivalence (see Tabuada [37, Definition 5.1]).

Definition 2.11 Let $\mathcal{A}$ be a $\mathcal{C}$-category. A right $\mathcal{A}$-module is a $\mathcal{C}$-functor from $\mathcal{A}$ to $\mathcal{C}$.

Remark 2.12 If the model category $\mathcal{C}$ is cofibrantly generated, the category $\mathcal{A}$-Mod of right $\mathcal{A}$-modules is naturally endowed with the projective model structure for which the weak equivalences and the fibrations are defined objectwise, see Hirschhorn [20, Theorem 11.6.1]. Notice that when $\mathcal{C}$ is a stable model category, the homotopy category $\mathrm{Ho}(\mathcal{A}-\mathrm{Mod})$ is triangulated.

We finish this section with some useful criteria:

Proposition 2.13 (see Dugger [7, A.3]) Let

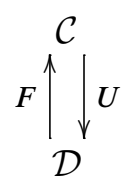

be an adjunction between Quillen model categories. If the right adjoint functor $U$ preserves trivial fibrations and fibrations between fibrant objects, then the adjunction $(F, U)$ is a Quillen adjunction.

Proposition 2.14 (see Hovey [21, Corollary 1.3.16]) Let

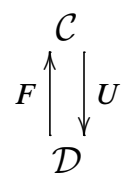

be a Quillen adjunction between model categories. The adjunction $(F, U)$ is a Quillen equivalence if and only if 
(a) the right adjoint $U$ reflects weak equivalences between fibrant objects and

(b) for every cofibrant object $X \in \mathcal{D}$, the composed morphism

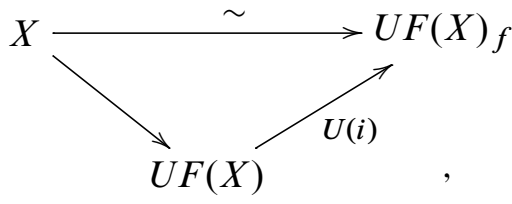

is a weak equivalence in $\mathcal{D}$, where $i: F(X) \rightarrow F(X)_{f}$ denotes a fibrant resolution of $F(X)$ in $\mathcal{C}$.

Throughout the paper the adjunctions are displayed vertically with the left, resp. right, adjoint on the left-hand side, resp. right-hand side.

\section{Homotopy theory of generalized spectral categories}

Hovey developed in [22] a homotopy theory of symmetric spectra in general Quillen model categories. Starting from a "well-behaved" monoidal model category $\mathcal{C}$ and a cofibrant object $K$ in $\mathcal{C}$, Hovey constructed the stable monoidal model category $\mathrm{Sp}^{\Sigma}(\mathcal{C}, K)$ of $K$-symmetric spectra over $\mathcal{C}$, see [22, Definition 7.2].

In this Section, starting from a "well-behaved" monoidal model category $\mathcal{C}$, a cofibrant object $K$ in $\mathcal{C}$ and a Quillen model structure on $\mathcal{C}$-Cat (see Notation 2.2), we construct a Quillen model structure on $\mathrm{Sp}^{\Sigma}(\mathcal{C}, K)-$ Cat, see Theorem 3.46. Our construction, which is inspired by the model structure on spectral categories (see Tabuada [37, Theorem 5.10]), is performed in two steps:

- First, we lift the model structure on $\mathcal{C}$-Cat to $\mathrm{Sp}^{\Sigma}(\mathcal{C}, K)-$ Cat along a natural adjunction (see Theorem 3.19) and obtain a model structure whose weak equivalences are the levelwise quasi-equivalences, see Definition 3.14.

- Then, using the generating trivial cofibrations of the stable model structure on $\mathrm{Sp}^{\Sigma}(\mathcal{C}, K)$, we construct a functor (see Section 3.9)

$$
Q: \operatorname{Sp}^{\Sigma}(\mathcal{C}, K)-\mathrm{Cat} \longrightarrow \mathrm{Sp}^{\Sigma}(\mathcal{C}, K)-\mathrm{Cat},
$$

which we use to localize the levelwise model structure, see Theorem 3.46. We obtain finally a model structure on $\operatorname{Sp}^{\Sigma}(\mathcal{C}, K)$-Cat, whose weak equivalences are the stable quasi-equivalences, see Definition 3.32. 


\subsection{Conditions on $\mathcal{C}$ and $K$}

Let $\left(\mathcal{C},-\otimes_{\mathcal{C}}-, \mathbf{1}_{\mathcal{C}}\right)$ be a left proper, cellular, cofibrantly generated, symmetric monoidal model category and $K$ a cofibrant object in $\mathcal{C}$. Under these conditions, Hovey constructed in [22, Section 7] the category $\operatorname{Sp}^{\Sigma}(\mathcal{C}, K)$ of $K$-symmetric spectra over $\mathcal{C}$. We will not review in here the classical notions related to $K$-symmetric spectra such as $\Omega$-spectrum, level equivalence, level fibration, stable equivalence and stable fibration. The reader unfamiliar with this language is invited to consult Hovey's paper [22].

We assume that $\mathcal{C}$ and $K$ satisfy the following extra natural conditions:

(C1) The Quillen model structure on $\mathcal{C}$ is right proper.

(C2) The stable model structure on $\operatorname{Sp}^{\Sigma}(\mathcal{C}, K$ ) (see Hovey [22, Definition 8.7]) is right proper.

(C3) The model structure on $\mathcal{C}$ satisfies the monoid axiom (see Schwede and Shipley [29, Definition 3.3]).

(C4) The stable model structure on $\operatorname{Sp}^{\Sigma}(\mathcal{C}, K)$ satisfies the monoid axiom.

(C5) The domains of the morphisms in the sets $I_{\mathcal{C}}$ and $J_{\mathcal{C}}$ (see Notation 2.3) are sequentially small.

(C6) The identity $\mathbf{1}_{\mathcal{C}}$ is a domain or codomain of an element of $I_{\mathcal{C}}$.

\section{Example 3.1}

(0) If we consider for $\mathcal{C}$ the category $s$ Set. of pointed simplicial sets, endowed with the model structure described by Hirschhorn in [20, Theorem 7.10.13] and for $K$ the pointed simplicial set $S^{1}$, then conditions (C1)-(C6) are satisfied: condition (C3) is proved by Schwede and Shipley in [29, Section 5] and condition (C4) is proved by Hovey, Shipley and Smith in [23, Section 5.4]. Conditions (C1), (C2), (C5) and (C6) are verified by construction. In this case, as it is mentioned by Hovey [22, Section 7], the category $\operatorname{Sp}^{\Sigma}\left(s\right.$ Set. $\left._{\bullet}, S^{1}\right)$ identifies with the classical category of symmetric spectra (see Hovey, Shipley and Smith [23]).

(1) If we consider for $\mathcal{C}$ the category $\mathbf{C h}$ of chain complexes, endowed with the model structure described by Hovey in [21, Theorem 2.3.11] and for $K$ the chain complex $\mathbb{Z}[1]$ (which contains a single copy of $\mathbb{Z}$ in dimension 1 ), then conditions (C1)-(C6) are satisfied: condition (C3) is proved by Shipley in [32, Proposition 3.1] and condition (C4) is proved by Shipley in [32, Proposition 2.9]. Conditions (C1), (C2), (C5) and (C6) are verified by construction. 
(2) If we consider for $\mathcal{C}$ the category $\mathbf{C h}_{\geq 0}$ of positively graded chain complexes, endowed with the model structure described by Goerss and Jardine in [15, Section III-2] and for $K$ the positively graded chain complex $\mathbb{Z}[1]$, then as in the previous example conditions $(\mathrm{C} 1)-(\mathrm{C} 6)$ are satisfied.

(3) If we consider for $\mathcal{C}$ the category $s \mathbf{A b}$ of simplicial abelian groups, endowed with the model structure described by Goerss and Jardine in [15, Theorem 2.8III] and for $K$ the simplicial abelian group $\widetilde{\mathbb{Z}}\left(S^{1}\right)$ (where $S^{1}=\Delta[1] / \partial \Delta[1]$ and $\widetilde{\mathbb{Z}}\left(S^{1}\right)_{n}$ is the free abelian group on the non-basepoint $n$-simplexes in $S^{1}$ ), then conditions $(\mathrm{C} 1)-(\mathrm{C} 6)$ are satisfied: conditions $(\mathrm{C} 3)$ and $(\mathrm{C} 4)$ are proved by Shipley in [32, Corollary 3.4] and conditions (C1), (C2), (C5) and (C6) are verified by construction.

Remark 3.2 In order to elucidate the reader, we now mention where conditions (C1)(C6) are used in the proof of Theorem 3.46. By condition (C1) the model structure on $\mathcal{C}$ is right proper, which implies right properness of the level model structure on $\mathrm{Sp}^{\Sigma}(\mathcal{C}, K)$, see Hovey [22, Theorem 8.3]. This will be used in the proof of Proposition 3.29. Since the stable model structure on $\operatorname{Sp}^{\Sigma}(\mathcal{C}, K)$ is obtained by localizing the levelwise one, it doesn't necessarily remains right proper. Therefore we assume it by requiring condition (C2). This will be used in the proof of Theorem 3.46(A3). By condition (C3) the model structure on $\mathcal{C}$ satisfies the monoid axiom, which implies that the level model structure on $\operatorname{Sp}^{\Sigma}(\mathcal{C}, K)$ (see Hovey [22, Section 8]) satisfies also the monoid axiom. This will be used in the proof of Lemma 3.24 and Proposition 3.30. Since, in general, it is not known if the stable model structure on $\operatorname{Sp}^{\Sigma}(\mathcal{C}, K)$ satisfies the monoid axiom (see Hovey [22, Section 8]), we assume it by requiring condition (C4). This will be used in the proof of Proposition 3.40. Since by condition (C5) the domains of the generating (trivial) cofibrations of $\mathcal{C}$ are sequentially small and the evaluation functors (see Hovey [22, Definition 7.3])

$$
\operatorname{Ev}_{n}: \operatorname{Sp}^{\Sigma}(\mathcal{C}, K) \longrightarrow \mathcal{C}, n \geq 0
$$

preserve filtered colimits, the domains of the generating (trivial) cofibrations of the level model structure on $\operatorname{Sp}^{\Sigma}(\mathcal{C}, K)$ are also sequentially small. This will be used in the proof of Theorem 3.19. Finally, condition (C6) will be used in the proof of Proposition 3.39.

\subsection{Conditions on $\mathcal{C}-$ Cat}

Joyal and Tierney constructed in [24] a natural model structure on the category of small categories. The weak equivalences are the equivalences of categories, the cofibrations are the functors which induce an injection on the object sets and the fibrations are the 
isofibrations, that is, the functors $F: \mathcal{A} \rightarrow \mathcal{B}$ such that for every object $x$ in $\mathcal{A}$ and every isomorphism $f: F x \stackrel{\sim}{\rightarrow} y$ in $\mathcal{B}$ there is an object $y^{\prime}$ in $\mathcal{A}$ and an isomorphism $g: x \stackrel{\sim}{\rightarrow} y^{\prime}$ in $\mathcal{A}$ such that $F(g)=f$. Hence, the following notion is quite natural:

Definition 3.3 A $\mathcal{C}$-functor $F: \mathcal{A} \rightarrow \mathcal{B}$ is a fibration if

(1) for all objects $x, y \in \mathcal{A}$, the morphism

$$
F(x, y): \mathcal{A}(x, y) \longrightarrow \mathcal{B}(F x, F y)
$$

is a fibration in $\mathcal{C}$ and

(2) the induced functor $[F]:[\mathcal{A}] \rightarrow[\mathcal{B}]$ (see Remark 2.7) is a Joyal-Tierney's isofibration [24].

We assume that if we take for weak equivalences the $\mathcal{C}$-functors of Definition 2.8 and for fibrations the $\mathcal{C}$-functors of Definition 3.3, then the category $\mathcal{C}$-Cat is endowed with a Quillen model structure. In Example 3.10 we will show that this is the case in all the familiar examples.

In order to construct a model structure on $\mathrm{Sp}^{\Sigma}(\mathcal{C}, K)-$ Cat, we assume moreover that the model structure on $\mathcal{C}$-Cat is cofibrantly generated with generating (trivial) cofibrations defined as follows:

\subsection{Generating cofibrations}

Recall from Appendix $\mathrm{C}$ the construction of the functor

$$
U: \mathcal{C} \longrightarrow \mathcal{C}-\text { Cat . }
$$

It sends an object $X \in \mathcal{C}$ to the $\mathcal{C}$-category $U(X)$, with two objects 1 and 2 such that $U(X)(1,1)=U(X)(2,2)=\mathbf{1}_{\mathcal{C}}, U(X)(1,2)=X$ and $U(X)(2,1)=0$, where 0 denotes the initial object in $\mathcal{C}$. Composition is naturally defined (notice that 0 acts as a zero with respect to $-\otimes_{\mathcal{C}}-$ since the bi-functor $-\otimes_{\mathcal{C}}-$ preserves colimits in each of its variables).

Definition 3.4 The set $I$ of generating cofibrations on $\mathcal{C}$-Cat consist of

- the $\mathcal{C}$-functors obtained by applying the functor $U$ to the set $I_{\mathcal{C}}$ of generating cofibrations on $\mathcal{C}$ and

- the $\mathcal{C}$-functor

$$
\varnothing \longrightarrow \underline{\mathbf{1}}_{\mathcal{C}}
$$

from the empty $\mathcal{C}$-category $\varnothing$ (which is the initial object in $\mathcal{C}$-Cat) to the $\mathcal{C}$-category $\underline{\mathbf{1}}_{\mathcal{C}}$ with one object $*$ and endomorphism ring $\mathbf{1}_{\mathcal{C}}$. 
Remark 3.5 Notice that the class of trivial fibrations in $\mathcal{C}$-Cat (that is, the class $I-$ inj) has a very simple description. A $\mathcal{C}$-functor $F: \mathcal{A} \rightarrow \mathcal{B}$ is a trivial fibration if

- for all objects $x, y \in \mathcal{A}$, the morphism

$$
F(x, y): \mathcal{A}(x, y) \rightarrow \mathcal{B}(F x, F y)
$$

is a trivial fibration in $\mathcal{C}$ and

- the $\mathcal{C}$-functor $F$ induces a surjective map on the object sets.

\subsection{Generating trivial cofibrations}

Recall from Remark A.2 that given a set $I$, the category $\mathcal{C}^{I}$-Cat (see Notation 2.2) carries a cofibrantly generated Quillen model structure, whose weak equivalences, resp. fibrations, are the morphisms $F: \mathcal{A} \rightarrow \mathcal{B}$ such that

$$
F(x, y): \mathcal{A}(x, y) \longrightarrow \mathcal{B}(x, y), \quad x, y \in \mathcal{A}
$$

is a weak equivalence, resp. a fibration, in $\mathcal{C}$.

Notation 3.6 If $\mathcal{A}$ is a $\mathcal{C}$-category and $x$ is an object of $\mathcal{A}$, we denote by $x^{*} \mathcal{A}$ the full $\mathcal{C}$-subcategory of $\mathcal{A}$ whose set of objects is $\{x\}$.

Definition 3.7 The set $J:=J^{\prime} \cup J^{\prime \prime}$ of generating trivial cofibrations on $\mathcal{C}$-Cat consists of

- the set $J^{\prime}$ of $\mathcal{C}$-functors obtained by applying the functor $U$ to the set $J_{\mathcal{C}}$ of generating trivial cofibrations on $\mathcal{C}$ and

- a fixed set $J^{\prime \prime}$ of $\mathcal{C}$-functors

$$
V_{\mathcal{H}}: \underline{\mathbf{1}}_{\mathcal{C}} \longrightarrow \mathcal{H}
$$

where $\mathcal{H}$ is a $\mathcal{C}$-category with two objects 1 and 2 and $V_{\mathcal{H}}$ is the $\mathcal{C}$-functor which sends the object $*$ to 1 .

Remark 3.8 In Example 3.10 we will show that all the familiar examples are endowed with model structures of this kind. In fact, any attempt to construct sets of generating (trivial) cofibrations for the model structure on $\mathcal{C}$-Cat (with respect to Definitions 2.8 and 3.3) will naturally led to the sets of Definitions 3.4 and 3.7. The difficult step will always be the construction of the $\mathcal{C}$-categories $\mathcal{H}$. Roughly, they correspond to "intervals" in this homotopy theory: a $\mathcal{C}$-functor from $\mathcal{H}$ to a $\mathcal{C}$-category $\mathcal{A}$ should correspond to the choice on an isomorphism in $[\mathcal{A}]$ and condition 2) of Definition 3.3 should correspond to the right lifting property with respect to the set $J^{\prime \prime}$. 
We assume also the following condition:

(H) The $\mathcal{C}$-categories with a single object $1^{*} \mathcal{H}$ (see Notation 3.6) are cofibrant in $\mathcal{C}^{\{1\}}$-Cat.

Remark 3.9 Condition $(\mathrm{H})$, which is verified in all the familiar examples, will be used in the proof of Proposition 3.25 to show that certain pushouts behave correctly.

\section{Example 3.10}

(0) In Example 3.1(0) consider the category $\mathcal{C}$-Cat endowed with the model structure described by Bergner in [1, Theorem 1.1] (lifted along the natural adjunction between simplicial sets and pointed simplicial sets, see Tabuada [37, Section 2]). The set $J^{\prime \prime}$ consists of the simplicial functors $\{x\}_{+} \rightarrow \mathcal{H}_{+}$, see [1, Section 1]. The fibrations are those of Definition 3.3 (see [1, Section 1]) and condition (H) is proved by Tabuada in [37, Lemma 3.3]. In this case the Quillen model structure of Theorem 3.46 identifies with the one constructed by Tabuada in [37, Theorem 5.10].

(1) In Example 3.1(1) consider the category $\mathcal{C}$-Cat endowed with the model structure described by Tabuada in [35, Theorem 1.8]. The set $J^{\prime \prime}$ consists of a single $\mathrm{dg}$ functor $F: \mathcal{A} \rightarrow \mathcal{K}$, see [35, Section 1.3]. The fibrations are those of Definition 3.3 (see Tabuada [35, Proposition 1.13]) and condition $(\mathrm{H})$ is verified by construction.

(2) In Example 3.1(2) consider the category $\mathcal{C}$-Cat endowed with the model structure described by Tabuada in [38, Theorem 4.7]. The set $J^{\prime \prime}$ consists also of a single dg functor $F: \mathcal{A} \rightarrow \mathcal{K}$ and as in the previous example the fibrations are those of Definition 3.3 and condition $(\mathrm{H})$ is verified by construction.

(3) In Example 3.1(3) consider the category $\mathcal{C}$-Cat endowed with the Quillen model structure described by Tabuada in [38, Theorem 5.10]. The set $J^{\prime \prime}$ consists of a single simplicial abelian functor $L(F): L(\mathcal{A}) \rightarrow L(\mathcal{K})$, see [38, Section 5.2]. The fibrations are those of Definition 3.3 (see Tabuada [38, Section 5.6]) and condition $(\mathrm{H})$ is verified by construction.

From now on and until the end of this Section, we assume that $\mathcal{C}, K$ and $\mathcal{C}$-Cat satisfy conditions (C1)-(C6) and condition (H).

Lemma 3.11 $A \mathcal{C}$-category $\mathcal{A}$ is fibrant if and only if for all objects $x, y \in \mathcal{A}$, the object $\mathcal{A}(x, y)$ is fibrant in $\mathcal{C}$. 
Proof Notice that the terminal object $\bullet$ in $\mathcal{C}$-Cat consists of the $\mathcal{C}$-category with a single object and endomorphism ring the terminal object of $\mathcal{C}$. This implies that the unique $\mathcal{C}$-functor $\mathcal{A} \rightarrow \bullet$ clearly satisfies condition (2) of Definition 3.3. By condition (1) of Definition 3.3 the proof is finished.

Proposition 3.12 The model structure on $\mathcal{C}-$ Cat is right proper.

Proof Consider the following pullback square in $\mathcal{C}-$ Cat

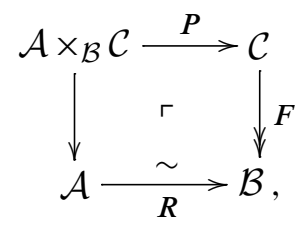

where $F$ is a fibration and $R$ is a weak equivalence. We need to show that $P$ is also a weak equivalence. Notice that pullbacks in $\mathcal{C}$-Cat are calculated at the level of objects and at the level of morphisms. Since $R$ satisfies condition (WE1) and $F$ satisfies condition (1) of Definition 3.3, we conclude by condition (C1) that $P$ satisfies also condition (WE1) By Remark 2.9 it is enough to show that $P$ satisfies condition (WE2). Consider an object $c$ in $\mathcal{C}$ and its image $F(c)$ in $\mathcal{B}$. Since $R$ is a weak equivalence, the functor $[R]$ is essentially surjective and so there exists an object $a \in \mathcal{A}$ together with an isomorphism $F(c) \stackrel{\sim}{\rightarrow} R(a)$ in $[\mathcal{B}]$. Since $F$ is a fibration, there exists an object $b$ in $\mathcal{C}$ and an isomorphism $c \stackrel{\sim}{\rightarrow} b$ in $[\mathcal{C}]$ such that $F(b)=R(a)$. This implies that the pullback of $a$ with $b$ under $R(a)$ is sent by $P$ to $b$ and so the proof is finished.

\subsection{Levelwise quasi-equivalences}

In this Section we consider the level model structure on $\operatorname{Sp}^{\Sigma}(\mathcal{C}, K)$, see Hovey [22, Theorem 8.3]. Recall from [22, Definition 7.2] that the category $\operatorname{Sp}^{\Sigma}(\mathcal{C}, K)$ of $K-$ symmetric spectra over $\mathcal{C}$ is endowed with a symmetric monoidal structure $-\wedge-$ whose identity is the $K$-spectrum

$$
\mathbf{1}_{\mathrm{Sp}^{\Sigma}(\mathcal{C}, K)}=\left(\mathbf{1}_{\mathcal{C}}, K, K \otimes K, \ldots, K^{\otimes^{n}}, \ldots\right),
$$

where the permutation group $\Sigma_{n}$ acts on $K^{\otimes^{n}}$ by permutation. Moreover, as it is shown by Hovey in [22, Definition 7.3], we have an adjunction

$$
\begin{gathered}
\operatorname{Sp}^{\Sigma}(\mathcal{C}, K) \\
\left.\left.F_{0}\right|_{\mathcal{C}}\right|^{\mathrm{Ev}_{0}} \\
\end{gathered}
$$


where both adjoints are strong monoidal (see Definition 2.4). By Remark A.5, we obtain the following adjunction

$$
\begin{gathered}
\operatorname{Sp}^{\Sigma}(\mathcal{C}, K)-\mathrm{Cat} \\
\left.F_{0} \uparrow\right|_{\mathcal{E} v_{0}} \\
\mathcal{C}-\text { Cat }
\end{gathered}
$$

Remark 3.13 By Remark 3.2, the right adjoint functor $\mathrm{Ev}_{0}$ preserves filtered colimits.

We now construct a Quillen model structure on $\operatorname{Sp}^{\Sigma}(\mathcal{C}, K)$-Cat, whose weak equivalence are as follows.

Definition 3.14 $\operatorname{A~Sp}^{\Sigma}(\mathcal{C}, K)$-functor $F: \mathcal{A} \rightarrow \mathcal{B}$ is a levelwise quasi-equivalence if (L1) for all objects $x, y \in \mathcal{A}$, the morphism

$$
F(x, y): \mathcal{A}(x, y) \rightarrow \mathcal{B}(F x, F y)
$$

is a level equivalence in $\operatorname{Sp}^{\Sigma}(\mathcal{C}, K)$ and

(L2) the induced $\mathcal{C}$-functor

$$
\operatorname{Ev}_{0}(F): \operatorname{Ev}_{0}(\mathcal{A}) \rightarrow \operatorname{Ev}_{0}(\mathcal{B})
$$

is a weak equivalence (see Definition 2.8) in $\mathcal{C}-$ Cat.

Notation 3.15 We denote by $\mathcal{W}_{L}$ the class of levelwise quasi-equivalences.

Remark 3.16 If condition (L1) is verified, condition (L2) is equivalent to

$\left(\mathrm{L} 2^{\prime}\right)$ the induced functor (see Remark 2.7)

$$
\left[\operatorname{Ev}_{0}(F)\right]:\left[\operatorname{Ev}_{0}(\mathcal{A})\right] \longrightarrow\left[\operatorname{Ev}_{0}(\mathcal{B})\right]
$$

is essentially surjective.

We now define our sets of generating (trivial) cofibrations.

Definition 3.17 The set $I_{L}$ of generating cofibrations in $\operatorname{Sp}^{\Sigma}(\mathcal{C}, K)$-Cat consists of

- the set $I_{L}^{\prime}$ of $\mathrm{Sp}^{\Sigma}(\mathcal{C}, K)$-functors obtained by applying the functor $U$ (see Definition C.1) to the set $I_{\mathrm{Sp}^{\Sigma}(\mathcal{C}, K)}$ of generating cofibrations for the level model structure on $\operatorname{Sp}^{\Sigma}(\mathcal{C}, K)$ (see Hovey [22, Theorem 8.2]) and 
- the $\mathrm{Sp}^{\Sigma}(\mathcal{C}, K)$-functor

$$
\varnothing \longrightarrow \underline{\mathbf{1}_{\mathrm{Sp}^{\Sigma}(\mathcal{C}, K)}}
$$

from the empty $\operatorname{Sp}^{\Sigma}(\mathcal{C}, K)$-category $\varnothing$ (which is the initial object) to the $\mathrm{Sp}^{\Sigma}(\mathcal{C}, K)$-category $\mathbf{1}_{\mathrm{Sp}^{\Sigma}(\mathcal{C}, K)}$ with one object $*$ and endomorphism ring $\mathbf{1}_{\mathrm{Sp}^{\Sigma}(\mathcal{C}, K)}$.

Definition 3.18 The set $J_{L}:=J_{L}^{\prime} \cup J_{L}^{\prime \prime}$ of generating trivial cofibrations in the category $\mathrm{Sp}^{\Sigma}(\mathcal{C}, K)-$ Cat consists of

- the set $J_{L}^{\prime}$ of $\operatorname{Sp}^{\Sigma}(\mathcal{C}, K)$-functors obtained by applying the functor $U$ to the set $J_{\mathrm{Sp}^{\Sigma}(\mathcal{C}, K)}$ of trivial generating cofibrations for the level model structure on $\mathrm{Sp}^{\Sigma}(\mathcal{C}, K)$ and

- the set $J_{L}^{\prime \prime}$ of $\operatorname{Sp}^{\Sigma}(\mathcal{C}, K)$-functors obtained by applying the functor $F_{0}$ to the set $J^{\prime \prime}$ of trivial generating cofibrations (see Definition 3.7) in $\mathcal{C}$-Cat.

Theorem 3.19 If we let $\mathcal{M}$ be the category $\operatorname{Sp}^{\Sigma}(\mathcal{C}, K)-C a t, W$ be the class $\mathcal{W}_{L}, I$ be the set $I_{L}$ of Definition 3.17 and $J$ the set $J_{L}$ of Definition 3.18, then the conditions of the recognition Theorem (see Hovey [21, Theorem 2.1.19]) are satisfied. Thus, the category $\mathrm{Sp}^{\Sigma}(\mathcal{C}, K)-C a t$ admits a cofibrantly generated Quillen model structure whose weak equivalences are the levelwise quasi-equivalences.

\subsection{Proof of Theorem 3.19}

Observe that the category $\mathrm{Sp}^{\Sigma}(\mathcal{C}, K)-$ Cat is complete and cocomplete and that the class $\mathcal{W}_{L}$ satisfies the two out of three axiom and is stable under retracts. Since by condition (C5) and Remark 3.2 the domains of the generating (trivial) cofibrations in $\operatorname{Sp}^{\Sigma}(\mathcal{C}, K)$ are sequentially small, the same holds by Remark C.2 for the domains of $\operatorname{Sp}^{\Sigma}(\mathcal{C}, K)$-functors in the sets $I_{L}^{\prime}$ and $J_{L}^{\prime}$. By Remark 3.13, this also holds for the remaining $\mathrm{Sp}^{\Sigma}(\mathcal{C}, K)$-functors of the sets $I_{L}$ and $J_{L}$. This implies that the first three conditions of the recognition Theorem (see Hovey [21, Theorem 2.1.19]) are verified. We now prove that $J_{L}-\mathrm{inj} \cap \mathcal{W}_{L}=I_{L}$-inj. For this we introduce the following auxiliary class of $\operatorname{Sp}^{\Sigma}(\mathcal{C}, K)$-functors:

Definition 3.20 Let Surj be the class of $\operatorname{Sp}^{\Sigma}(\mathcal{C}, K)$-functors $F: \mathcal{A} \rightarrow \mathcal{B}$ such that (Sj1) for all objects $x, y \in \mathcal{A}$, the morphism

$$
F(x, y): \mathcal{A}(x, y) \rightarrow \mathcal{B}(F x, F y)
$$

is a trivial level fibration in $\operatorname{Sp}^{\Sigma}(\mathcal{C}, K)$ and 
$(\mathrm{Sj} 2)$ the $\mathrm{Sp}^{\Sigma}(\mathcal{C}, K)$-functor $F$ induces a surjective map on the object sets.

\section{Lemma 3.21 $I_{L}-i n j=$ Surj .}

Proof Notice that by adjunction (see Remark C.2), a $\operatorname{Sp}^{\Sigma}(\mathcal{C}, K)$-functor satisfies condition $(\mathrm{Sj} 1)$ if and only if it has the RLP with respect to the set $I_{L}^{\prime}$ of generating cofibrations. Clearly a $\mathrm{Sp}^{\Sigma}(\mathcal{C}, K)$-functor has the RLP with respect to $\varnothing \rightarrow \underline{\mathbf{1 p}^{\Sigma}(\mathcal{C}, K)}$ if and only if it satisfies condition $(\mathrm{Sj} 2)$.

\section{Lemma 3.22 $\operatorname{Surj}=J_{L}-i n j \cap \mathcal{W}_{L}$.}

Proof We prove first the inclusion $\subseteq$. Let $F: \mathcal{A} \rightarrow \mathcal{B}$ be a $\operatorname{Sp}^{\Sigma}(\mathcal{C}, K)$-functor which belongs to Surj. Clearly condition ( $\mathrm{Sj} 1)$ and $(\mathrm{Sj} 2)$ imply conditions (L1) and (L2), which shows that $F$ belongs to $\mathcal{W}_{L}$. Notice also that a $\operatorname{Sp}^{\Sigma}(\mathcal{C}, K)$-functor which satisfies condition $(\mathrm{Sj} 1)$ has the RLP with respect to the set $J_{L}^{\prime}$ of generating trivial cofibrations. It is then enough to show that $F$ has the RLP with respect to the set $J_{L}^{\prime \prime}$ of generating trivial cofibrations. By adjunction, this is equivalent to demand that the $\mathcal{C}$-functor $\operatorname{Ev}_{0}(F): \operatorname{Ev}_{0}(\mathcal{A}) \rightarrow \operatorname{Ev}_{0}(\mathcal{B})$ has the RLP with respect to the set $J^{\prime \prime}$ of generating trivial cofibrations in $\mathcal{C}$-Cat. Since $F$ satisfies conditions $(\mathrm{Sj} 1)$ and $(\mathrm{Sj} 2)$, Remark 3.9 implies that $\operatorname{Ev}_{0}(F)$ is a trivial fibration in $\mathcal{C}$-Cat and so the claim follows.

We now prove the inclusion $\supseteq$. Start by observing that a $\operatorname{Sp}^{\Sigma}(\mathcal{C}, K)$-functor satisfies condition $(\mathrm{Sj} 1)$ if and only if it satisfies condition (L1) and it has the RLP with respect to the set $J_{L}^{\prime}$ of generating trivial cofibrations. Now, let $F: \mathcal{A} \rightarrow \mathcal{B}$ be a $\operatorname{Sp}^{\Sigma}(\mathcal{C}, K)-$ functor which belongs to $J_{L}$-inj $\cap \mathcal{W}_{L}$. It is then enough to show that it satisfies condition $(\mathrm{Sj} 2)$. Since $F$ has the RLP with respect to the set $J_{L}$ of generating trivial cofibrations, the $\mathcal{C}$-functor $\operatorname{Ev}_{0}(F): \operatorname{Ev}_{0}(\mathcal{A}) \rightarrow \operatorname{Ev}_{0}(\mathcal{B})$ has the RLP with respect to the set $J$ of generating trivial cofibrations in $\mathcal{C}$-Cat. This implies that $\operatorname{Ev}_{0}(F)$ is a trivial fibration in $\mathcal{C}$-Cat and so by Remark 3.5, the $\mathcal{C}$-functor $\operatorname{Ev}_{0}(F)$ induces a surjective map on the object sets. Since $\operatorname{Ev}_{0}(F)$ and $F$ induce the same map on the object sets, the $\mathrm{Sp}^{\Sigma}(\mathcal{C}, K)$-functor $F$ satisfies condition $(\mathrm{Sj} 2)$.

We now characterize the class $J_{L^{-}}$inj.

Lemma 3.23 $A \operatorname{Sp}^{\Sigma}(\mathcal{C}, K)$-functor $F: \mathcal{A} \rightarrow \mathcal{B}$ has the $R L P$ with respect to the set $J_{L}$ of trivial generating cofibrations if and only if it satisfies

(F1) for all objects $x, y \in \mathcal{A}$, the morphism

$$
F(x, y): \mathcal{A}(x, y) \rightarrow \mathcal{B}(F x, F y)
$$

is a level fibration in $\operatorname{Sp}^{\Sigma}(\mathcal{C}, K)$ and 
(F2) the induced $\mathcal{C}$-functor

$$
\operatorname{Ev}_{0}(F): \operatorname{Ev}_{0}(\mathcal{A}) \rightarrow \operatorname{Ev}_{0}(\mathcal{B})
$$

is a fibration in the Quillen model structure on $\mathcal{C}-$ Cat.

Proof Observe that a $\operatorname{Sp}^{\Sigma}(\mathcal{C}, K)$-functor $F$ satisfies condition (F1) if and only if it has the RLP with respect to the set $J_{L}^{\prime}$ of generating trivial cofibrations. By adjunction, $F$ has the RLP with respect to the set $J_{L}^{\prime \prime}$ if and only if the $\mathcal{C}$-functor $\operatorname{Ev}_{0}(F)$ has the RLP with respect to the set $J^{\prime \prime}$. In conclusion, $F$ has the RLP with respect to the set $J_{L}$ if and only if it satisfies conditions (F1) and (F2) altogether.

Lemma 3.24 $J_{L}^{\prime}-\operatorname{cell} \subseteq \mathcal{W}_{L}$.

Proof Since the class $\mathcal{W}_{L}$ is stable under transfinite compositions (see Hirschhorn [20, Definition 10.2.2]) it is enough to prove the following: consider the pushout

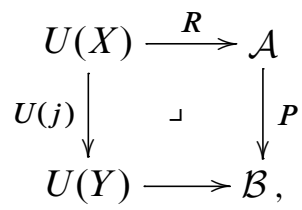

where $j$ belongs to the set $J_{\mathrm{Sp}^{\Sigma}(\mathcal{C}, K)}$ of generating trivial cofibrations on $\mathrm{Sp}^{\Sigma}(\mathcal{C}, K)$. We need to show that $P$ belongs to $\mathcal{W}_{L}$. Since the morphism $j: X \rightarrow Y$ is a trivial level cofibration in $\operatorname{Sp}^{\Sigma}(\mathcal{C}, K)$, Proposition C.3 and condition (C3) (see Remark 3.2) imply that the spectral functor $P$ satisfies condition (L1). Since $P$ induces the identity map on the object sets, condition (L2') is automatically verified and so $P$ belongs to $\mathcal{W}_{L}$.

Proposition 3.25 $J_{L}^{\prime \prime}-\mathrm{cell} \subseteq \mathcal{W}_{L}$.

Proof Since the class $\mathcal{W}_{L}$ is stable under transfinite compositions, it is enough to prove the following: consider the pushout

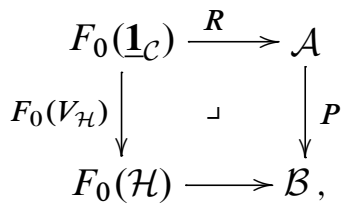


where $V_{\mathcal{H}}: \underline{\mathbf{1}}_{\mathcal{C}} \rightarrow \mathcal{H}$ belongs to the set $J^{\prime \prime}$ of generating trivial cofibrations in $\mathcal{C}$-Cat. We need to show that $P$ belongs to $\mathcal{W}_{L}$. We start by condition (L1). Factor the $\mathrm{Sp}^{\Sigma}(\mathcal{C}, K)$-functor $F_{0}\left(V_{\mathcal{H}}\right)$ as

$$
F_{0}\left(\underline{\mathbf{1}}_{\mathcal{C}}\right) \rightarrow 1^{*}\left(F_{0}(\mathcal{H})\right) \hookrightarrow F_{0}(\mathcal{H}),
$$

where $1^{*}\left(F_{0}(\mathcal{H})\right)$ is the full $\mathrm{Sp}^{\Sigma}(\mathcal{C}, K)$-subcategory of $F_{0}(\mathcal{H})$ whose set of objects is $\{1\}$ (see Notation 3.6). Consider the iterated pushout

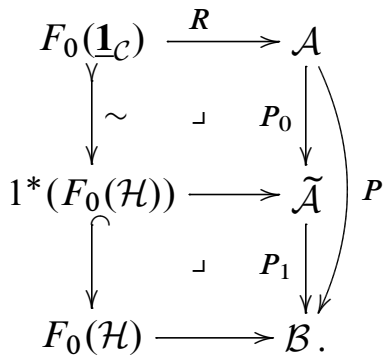

In the lower pushout, since $1^{*}\left(F_{0}(\mathcal{H})\right)$ is a full $\mathrm{Sp}^{\Sigma}(\mathcal{C}, K)$-subcategory of $F_{0}(\mathcal{H})$, the morphism

$$
\left(1^{*} F_{0}(\mathcal{H})\right)(1,1) \stackrel{\sim}{\longrightarrow} F_{0}(\mathcal{H})(1,1)
$$

is an isomorphism in $\operatorname{Sp}^{\Sigma}(\mathcal{C}, K)$. This implies by Stanculescu [33, Proposition 7.1] that for all objects $x, y \in \widetilde{\mathcal{A}}$, the morphism

$$
P_{1}(x, y): \widetilde{\mathcal{A}}(x, y) \stackrel{\sim}{\longrightarrow} \mathcal{B}\left(P_{1} x, P_{1} y\right)
$$

is an isomorphism in $\operatorname{Sp}^{\Sigma}(\mathcal{C}, K)$. In particular the $\mathrm{Sp}^{\Sigma}(\mathcal{C}, K)$-functor $P_{1}$ satisfies condition (L1). Let us now analyse the upper pushout. Since by condition $(H)$ the $\mathcal{C}$-category $1^{*} \mathcal{H}$ is cofibrant in $\mathcal{C}^{\{1\}}$-Cat, the morphism

$$
\underline{\mathbf{1}}_{\mathcal{C}} \stackrel{\sim}{\longrightarrow} 1^{*} \mathcal{H}
$$

is a trivial cofibration, which implies that so it is the morphism

$$
F_{0}\left(\underline{\mathbf{1}}_{\mathcal{C}}\right)>\stackrel{\sim}{\longrightarrow} F_{0}\left(1^{*}(\mathcal{H})\right)=1^{*}\left(F_{0}(\mathcal{H})\right)
$$

Now, let $\mathcal{O}$ denote the set of objects of $\mathcal{A}$ and denote by $\mathcal{O}^{\prime}$ the set of objects $\mathcal{O} \backslash R(1)$. Observe that $\tilde{\mathcal{A}}$ identifies with the following pushout in $\operatorname{Sp}^{\Sigma}(\mathcal{C}, K)^{\mathcal{O}}$-Cat (see Remark 
A.2).

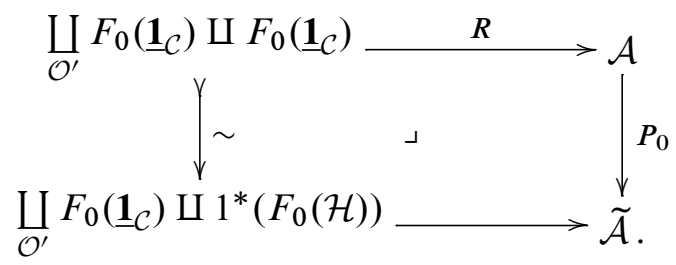

Since the left vertical arrow is a trivial cofibration so it is $P_{0}$. This shows that $P_{0}$ satisfies condition (L1) and so we conclude that the composed dg functor $P=P_{0} \circ P_{1}$ satisfies also condition (L1).

We now show that $P$ satisfies condition (L2'). Consider the following commutative square in Cat

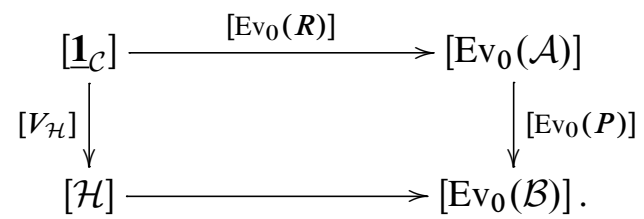

We need to show that the functor $\left[\operatorname{Ev}_{0}(P)\right]$ is essentially surjective. Notice that if in the previous diagram we restrict ourselves to the objects of each category $(\operatorname{obj}(-))$, we obtain the following co-cartesian square

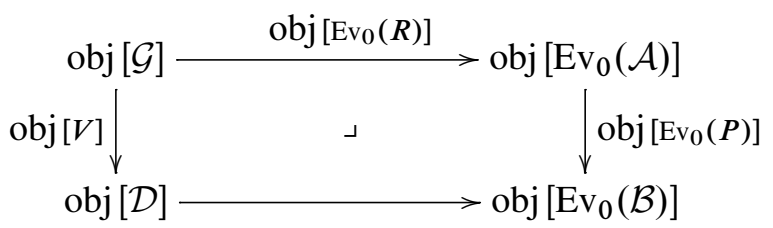

in Set. Since $V$ belongs to $J^{\prime \prime}$, the functor $[V]$ is essentially surjective. These facts imply, by a simple diagram chasing argument, that $\left[\operatorname{Ev}_{0}(P)\right]$ is also essentially surjective and so the $\mathrm{Sp}^{\Sigma}(\mathcal{C}, K)$-functor $P$ satisfies condition $\left(\mathrm{L}^{\prime}\right)$. In conclusion, $P$ satisfies condition (L1) and (L2') and so it belongs to $\mathcal{W}_{L}$.

We have shown that $J_{L}$-cell $\subseteq \mathcal{W}_{L}$ (Lemma 3.24 and Proposition 3.25) and that $I_{L}$-inj $=J_{L}$-inj $\cap \mathcal{W}_{L}$ (Lemmas 3.21 and 3.22). This implies that the last three conditions of the recognition Theorem (see Hovey [21, Theorem 2.1.19]) are satisfied. This finishes the proof of Theorem 3.19.

\subsection{Properties I}

Proposition 3.26 $A \operatorname{Sp}^{\Sigma}(\mathcal{C}, K)$-functor $F: \mathcal{A} \rightarrow \mathcal{B}$ is a fibration with respect to the model structure of Theorem 3.19, if and only if it satisfies conditions (F1) and (F2) of Lemma 3.23. 
Proof The proof follows from Lemma 3.23, since by the Recognition Theorem (see Hovey [21, Theorem 2.1.19]) the set $J_{L}$ is a set of generating trivial cofibrations.

Proposition 3.27 $A \operatorname{Sp}^{\Sigma}(\mathcal{C}, K)$-category $\mathcal{A}$ is fibrant with respect to the model structure of Theorem 3.19, if and only if for all objects $x, y \in \mathcal{C}$, the $K$-spectrum $\mathcal{A}(x, y)$ is levelwise fibrant in $\operatorname{Sp}^{\Sigma}(\mathcal{C}, K)$, see Hovey [22, Definition 8.1].

Proof It follows from Proposition 3.26 and Lemma 3.11.

Remark 3.28 By Proposition 3.26 and Remarks 3.5 and 3.9 we have a Quillen adjunction

$$
\begin{gathered}
\operatorname{Sp}^{\Sigma}(\mathcal{C}, K)-\mathrm{Cat} \\
\left.F_{0} \uparrow\right|_{\mathcal{C}} \mathrm{Ev}_{0} \\
\mathcal{C}-\mathrm{Cat} .
\end{gathered}
$$

Proposition 3.29 The Quillen model structure on $\operatorname{Sp}^{\Sigma}(\mathcal{C}, K)-C a t$ of Theorem 3.19 is right proper.

Proof Consider the following pullback square in $\operatorname{Sp}^{\Sigma}(\mathcal{C}, K)-$ Cat

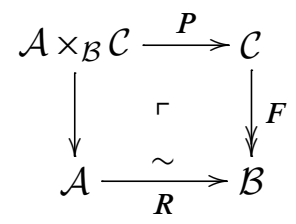

with $R$ a levelwise quasi-equivalence and $F$ a fibration. We need to show that $P$ is a levelwise quasi-equivalence. Notice that pullbacks in $\mathrm{Sp}^{\Sigma}(\mathcal{C}, K)-$ Cat are calculated at the level of objects and at the level of morphisms. Since by condition (C1) (see Remark 3.2) the level model structure on $\operatorname{Sp}^{\Sigma}(\mathcal{C}, K)$ is right proper and $F$ satisfies condition (F1), the $\mathrm{Sp}^{\Sigma}(\mathcal{C}, K)$-functor $P$ satisfies condition (L1). By applying the functor $\mathrm{Ev}_{0}$ to the previous square, we obtain a pullback square in $\mathcal{C}-$ Cat

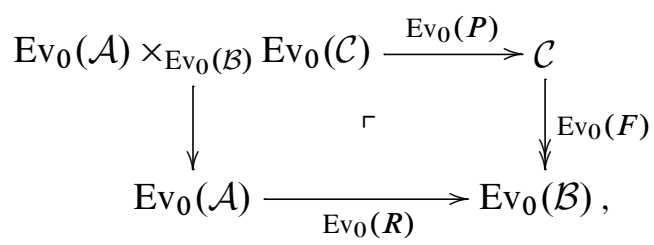

with $\mathrm{Ev}_{0}(R)$ a weak equivalence and $\mathrm{Ev}_{0}(F)$ a fibration. By Proposition 3.12 we conclude that $P$ satisfies condition (L2). 
Proposition 3.30 Let $\mathcal{A}$ be a cofibrant $\operatorname{Sp}^{\Sigma}(\mathcal{C}, K)$-category. Then for all objects $x, y \in \mathcal{A}$, the $K$-spectrum $\mathcal{A}(x, y)$ is cofibrant in the level model structure on $\mathrm{Sp}^{\Sigma}(\mathcal{C}, K)$.

Proof The model structure of Theorem 3.19 is cofibrantly generated and so every cofibrant object in $\mathrm{Sp}^{\Sigma}(\mathcal{C}, K)$-Cat is a retract of a $I_{L}$-cell complex, see Hirschhorn [20, Corollary 11.2.2]. Since cofibrations are stable under filtered colimits, it is enough to prove the Proposition for pushouts along a generating cofibration. Let $\mathcal{A}$ be a $\mathrm{Sp}^{\Sigma}(\mathcal{C}, K)$-category such that $\mathcal{A}(x, y)$ is cofibrant for all objects $x, y \in \mathcal{A}$ :

- Consider the following pushout

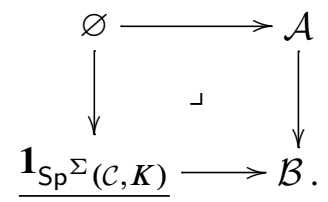

Notice that $\mathcal{B}$ is obtained from $\mathcal{A}$, by simply introducing a new object. It is then clear that for all objects $x, y \in \mathcal{B}$, the $K$-spectrum $\mathcal{B}(x, y)$ is cofibrant in the level model structure on $\mathrm{Sp}^{\Sigma}(\mathcal{C}, K)$.

- Now, consider the following pushout

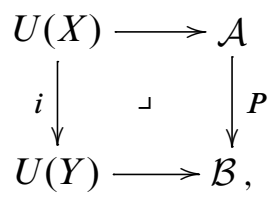

where $i: X \rightarrow Y$ belongs to the set $I_{\mathrm{Sp}^{\Sigma}(\mathcal{C}, K)}$ of generating cofibrations of $\mathrm{Sp}^{\Sigma}(\mathcal{C}, K)$. Notice that $\mathcal{A}$ and $\mathcal{B}$ have the same set of objects and $P$ induces the identity map on the object sets. Since $i: X \rightarrow Y$ is a cofibration, Proposition C.4 and condition (C3) (see Remark 3.2) imply that the morphism

$$
P(x, y): \mathcal{A}(x, y) \longrightarrow \mathcal{B}(x, y)
$$

is still a cofibration. Since $I$-cell complexes in $\operatorname{Sp}^{\Sigma}(\mathcal{C}, K)-$ Cat are built of $\varnothing$ (the initial object), the Proposition is proven.

Lemma 3.31 The functor

$$
U: \operatorname{Sp}^{\Sigma}(\mathcal{C}, K) \longrightarrow \operatorname{Sp}^{\Sigma}(\mathcal{C}, K)-C a t \quad \text { (see Definition C.1) }
$$

sends cofibrations to cofibrations. 
Proof The model structure of Theorem 3.19 is cofibrantly generated and so any cofibrant object in $\operatorname{Sp}^{\Sigma}(\mathcal{C}, K)$-Cat is a retract of a (possibly infinite) composition of pushouts along the generating cofibrations. Since the functor $U$ preserves retractions, colimits and send the generating cofibrations to (generating) cofibrations (see Definition 3.17) the proof is finished.

\subsection{Stable quasi-equivalences}

In this Section we consider the stable model structure on $\mathrm{Sp}^{\Sigma}(\mathcal{C}, K)$, see Hovey [22, Definition 8.7]. Recall from [22, Definition 7.2] that since $\mathcal{C}$ is a closed symmetric monoidal category, the category $\operatorname{Sp}^{\Sigma}(\mathcal{C}, K)$ is tensored, co-tensored and enriched over $\mathcal{C}$. In what follows, we will denote by $\underline{\operatorname{Hom}}(-,-)$ this $\mathcal{C}$-enrichment and by $\operatorname{Hom}_{\mathcal{C}}(-,-)$ the internal Hom-object in $\mathcal{C}$. We now construct a Quillen model structure on $\mathrm{Sp}^{\Sigma}(\mathcal{C}, K)-$ Cat, whose weak equivalences are as follows.

Definition 3.32 $\mathrm{A} \mathrm{Sp}^{\Sigma}(\mathcal{C}, K)$-functor $F: \mathcal{A} \rightarrow \mathcal{B}$ is a stable quasi-equivalence if

(S1) for all objects $x, y \in \mathcal{A}$, the morphism

$$
F(x, y): \mathcal{A}(x, y) \longrightarrow \mathcal{B}(F x, F y)
$$

is a stable equivalence in $\operatorname{Sp}^{\Sigma}(\mathcal{C}, K)$ and

(S2) the induced functor (see Remark 2.7)

$$
[F]:[\mathcal{A}] \longrightarrow[\mathcal{B}]
$$

is an equivalence of categories.

Remark 3.33 Notice that Definition 3.32 is a particular case of the general Definition 2.8, where we have taken $\operatorname{Sp}^{\Sigma}(\mathcal{C}, K)$ for the base symmetric monoidal model category.

Notation 3.34 We denote by $\mathcal{W}_{S}$ the class of stable quasi-equivalences.

\section{9 $Q$-functor}

We now construct a functor

$$
Q: \operatorname{Sp}^{\Sigma}(\mathcal{C}, K)-\mathrm{Cat} \longrightarrow \mathrm{Sp}^{\Sigma}(\mathcal{C}, K)-\mathrm{Cat}
$$

and a natural transformation $\eta: \operatorname{Id} \rightarrow Q$, from the identity functor on $\operatorname{Sp}^{\Sigma}(\mathcal{C}, K)$-Cat to $Q$. Recall from Hovey [22, Definition 8.7] the construction of the following set of morphisms in $\operatorname{Sp}^{\Sigma}(\mathcal{C}, K)$ :

$$
\mathcal{S}:=\left\{F_{n+1}(C \otimes K) \stackrel{\zeta_{n}^{C}}{\longrightarrow} F_{n} C \mid n \geq 0\right\},
$$


where $C$ runs through the domains and codomains of the generating cofibrations of $\mathcal{C}$ and $\zeta_{n}^{C}$ is the adjoint to the map

$$
C \otimes K \longrightarrow \operatorname{Ev}_{n+1} F_{n}(C)=\Sigma_{n+1} \times(C \otimes K)
$$

corresponding to the identity of $\Sigma_{n+1}$. For each element of $\mathcal{S}$, we consider a factorization

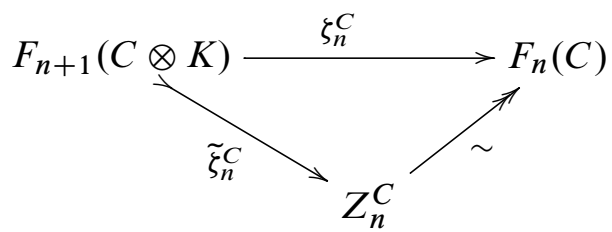

in the level model structure on $\mathrm{Sp}^{\Sigma}(\mathcal{C}, K)$. Recall from Hovey [22, Definition 8.7] that the stable model structure on $\operatorname{Sp}^{\Sigma}(\mathcal{C}, K)$ is the left Bousfield localization of the level model structure with respect to the morphisms $\zeta_{n}^{C}$ (or with respect to the morphisms $\left.\tilde{\zeta}_{n}^{C}\right)$.

Definition 3.35 Let $\widetilde{\mathcal{S}}$ be the following set of morphisms in $\operatorname{Sp}^{\Sigma}(\mathcal{C}, K)$ :

$$
i \otimes \widetilde{\zeta}_{n}^{C}: B \otimes F_{n+1}(C \otimes K) \coprod_{A \otimes F_{n+1}(C \otimes K)} A \otimes Z_{n}^{C} \longrightarrow B \otimes Z_{n}^{C},
$$

where $i: A \rightarrow B$ is a generating cofibration in $\mathcal{C}$.

Remark 3.36 Since $\operatorname{Sp}^{\Sigma}(\mathcal{C}, K)$ is a monoidal model category, the elements of the set $\tilde{\mathcal{S}}$ are cofibrations with cofibrant (and sequentially small) domains.

Definition 3.37 Let $\mathcal{A}$ be a $\operatorname{Sp}^{\Sigma}(\mathcal{C}, K)$-category. The functor

$$
Q: \mathrm{Sp}^{\Sigma}(\mathcal{C}, K)-\mathrm{Cat} \rightarrow \mathrm{Sp}^{\Sigma}(\mathcal{C}, K)-\mathrm{Cat}
$$

is obtained by the small object argument, using the set $J_{L}^{\prime} \cup U(\widetilde{\mathcal{S}})$ (see Definition C.1) to factorize the $\operatorname{Sp}^{\Sigma}(\mathcal{C}, K)$-functor $\mathcal{A} \longrightarrow \bullet$, where $\bullet$ denotes the terminal object in $\mathrm{Sp}^{\Sigma}(\mathcal{C}, K)-$ Cat.

Remark 3.38 We obtain in this way a functor $Q$ and a natural transformation $\eta$ : Id $\rightarrow$ $Q$. Notice also that $Q(\mathcal{A})$ has the same objects as $\mathcal{A}$, and the RLP with respect to the set $J_{L}^{\prime} \cup U(\widetilde{\mathcal{S}})$.

Proposition 3.39 Let $\mathcal{A}$ be a $\operatorname{Sp}^{\Sigma}(\mathcal{C}, K)$-category which has the right lifting property with respect to the set $J_{L}^{\prime} \cup U(\widetilde{\mathcal{S}})$. Then it satisfies the following condition: 
$(\Omega)$ for all objects $x, y \in \mathcal{A}$, the $K$-spectrum $\mathcal{A}(x, y)$ is an $\Omega$-spectrum, see Hovey [22, Definition 8.6].

Proof By Proposition 3.27, $\mathcal{A}$ has the RLP with respect to $J_{L}^{\prime}$ if and only if for all objects $x, y \in \mathcal{A}$, the $K$-spectrum $\mathcal{A}(x, y)$ is levelwise fibrant in $\operatorname{Sp}^{\Sigma}(\mathcal{C}, K)$.

Now, suppose that $\mathcal{A}$ has the RLP with respect to $U(\widetilde{\mathcal{S}})$. By adjunction we conclude that for all objects $x, y \in \mathcal{A}$, the induced morphism

$$
\underline{\operatorname{Hom}}\left(Z_{n}^{C}, \mathcal{A}(x, y)\right) \longrightarrow \underline{\operatorname{Hom}}\left(F_{n+1}(C \otimes K), \mathcal{A}(x, y)\right)
$$

is a trivial fibration in $\mathcal{C}$. Notice also that we have the following weak equivalences

$$
\begin{aligned}
\underline{\operatorname{Hom}}\left(Z_{n}^{C}, \mathcal{A}(x, y)\right) & \simeq \underline{\operatorname{Hom}}_{\left(F_{n}(C), \mathcal{A}(x, y)\right)} \\
& \simeq \underline{\operatorname{Hom}}_{\mathcal{C}}\left(C, \mathcal{A}(x, y)_{n}\right)
\end{aligned}
$$

and

$$
\begin{aligned}
\operatorname{Hom}\left(F_{n+1}(C \otimes K), \mathcal{A}(x, y)\right) & \simeq \underline{\operatorname{Hom}}_{\mathcal{C}}\left(C \otimes K, \mathcal{A}(x, y)_{n+1}\right) \\
& \simeq \underline{\operatorname{Hom}}_{\mathcal{C}}\left(C, \mathcal{A}(x, y)_{n+1}^{K}\right) .
\end{aligned}
$$

This implies that the induced morphisms in $\mathcal{C}$

$$
\underline{\operatorname{Hom}}_{\mathcal{C}}\left(C, \mathcal{A}(x, y)_{n}\right) \stackrel{\sim}{\longrightarrow} \underline{\operatorname{Hom}}_{\mathcal{C}}\left(C, \mathcal{A}(x, y)_{n+1}^{K}\right)
$$

are weak equivalences, where $C$ runs through the domains and codomains of the generating cofibrations of $\mathcal{C}$. By condition (C6), we obtain then the following weak equivalence

$$
\mathcal{A}(x, y)_{n} \simeq \underline{\operatorname{Hom}}_{\mathcal{C}}\left(\mathbf{1}_{\mathcal{C}}, \mathcal{A}(x, y)_{n}\right) \stackrel{\sim}{\longrightarrow} \underline{\operatorname{Hom}}_{\mathcal{C}}\left(\mathbf{1}_{\mathcal{C}}, \mathcal{A}(x, y)_{n+1}^{K}\right) \simeq \mathcal{A}(x, y)_{n+1}^{K} .
$$

In conclusion, for all objects $x, y \in \mathcal{A}$, the $K$-spectrum $\mathcal{A}(x, y)$ is an $\Omega$-spectrum.

Proposition 3.40 Let $\mathcal{A}$ be a $\mathrm{Sp}^{\Sigma}(\mathcal{C}, K)$-category. The $\mathrm{Sp}^{\Sigma}(\mathcal{C}, K)$-functor

$$
\eta_{\mathcal{A}}: \mathcal{A} \longrightarrow Q(\mathcal{A})
$$

is a stable quasi-equivalence.

Proof Notice that the elements of the set $J_{\mathrm{Sp}^{\Sigma}(\mathcal{C}, K)} \cup \widetilde{\mathcal{S}}$ are trivial cofibrations in the stable model structure on $\operatorname{Sp}^{\Sigma}(\mathcal{C}, K)$, see Hovey [22, Theorem 8.8]. Notice also that the stable model structure is monoidal (see [22, Theorem 8.11]) and that by condition (C4), it satisfies the monoid axiom. This implies, by Proposition C.3, that $\eta_{\mathcal{A}}$ satisfies condition (WE1) (see Definition 2.8). Since the $\operatorname{Sp}^{\Sigma}(\mathcal{C}, K)$-categories $Q(\mathcal{A})$ and $\mathcal{A}$ have the same set object sets, condition (WE2') is automatically verified. By Remark 3.33 the proof is finished. 


\subsection{Main Theorem}

Definition 3.41 $\operatorname{A~Sp}^{\Sigma}(\mathcal{C}, K)$-functor $F: \mathcal{A} \rightarrow \mathcal{B}$ is:

- a $Q$-weak equivalence if $Q(F)$ is a levelwise quasi-equivalence (see Definition 3.14).

- a cofibration if it is a cofibration in the model structure of Theorem 3.19.

- a $Q$-fibration if it has the RLP with respect to all cofibrations which are $Q$-weak equivalences.

Lemma 3.42 Let $\mathcal{A}$ be a $\operatorname{Sp}^{\Sigma}(\mathcal{C}, K)$-category which satisfies condition $(\Omega)$ of Proposition 3.39. Then the category $[\mathcal{A}]$ (see Remark 2.7 ) identifies naturally with the category $\left[\operatorname{Ev}_{0}(\mathcal{A})\right]$

Proof Recall from Hovey [22, Theorem 8.8] that an object in $\operatorname{Sp}^{\Sigma}(\mathcal{C}, K)$ is stably fibrant if and only if it is an $\Omega$-spectrum. Since the adjunction

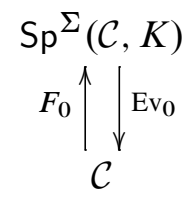

is strong monoidal, we have the following identifications

$$
\left[\mathbf{1}_{\mathrm{Sp}^{\Sigma}(\mathcal{C}, K)}, \mathcal{A}(x, y)\right] \simeq\left[\mathbf{1}_{\mathcal{C}}, \operatorname{Ev}_{0}(\mathcal{A}(x, y))\right], x, y \in \mathcal{A},
$$

which imply the Lemma.

Corollary 3.43 Let $F: \mathcal{A} \rightarrow \mathcal{B}$ be a $\operatorname{Sp}^{\Sigma}(\mathcal{C}, K)$-functor between $\operatorname{Sp}^{\Sigma}(\mathcal{C}, K)$-categories which satisfy condition $(\Omega)$. Then $F$ satisfies condition (WE2) if and only if $\operatorname{Ev}_{0}(F)$ satisfies condition (WE2).

Proposition 3.44 Let $F: \mathcal{A} \rightarrow \mathcal{B}$ be stable quasi-equivalence between $\mathrm{Sp}^{\Sigma}(\mathcal{C}, K)-$ categories which satisfy the condition $(\Omega)$. Then $F$ is a levelwise quasi-equivalence (see Definition 3.14).

Proof Since $F$ satisfies condition (WE1) and $\mathcal{A}$ and $\mathcal{B}$ satisfy condition $(\Omega$ ), Hirschhorn [20, Theorems 4.3.5 and 4.3.6] implies that $F$ satisfies condition (L1). By Corollary 3.43, the $\mathrm{Sp}^{\Sigma}(\mathcal{C}, K)$-functor $F$ satisfies condition (WE2) if and only if it satisfies condition (L2). 
Lemma 3.45 $A \operatorname{Sp}^{\Sigma}(\mathcal{C}, K)$-functor $F: \mathcal{A} \rightarrow \mathcal{B}$ is a $Q$-weak equivalence if and only if it is a stable quasi-equivalence.

Proof We have at our disposal a commutative square

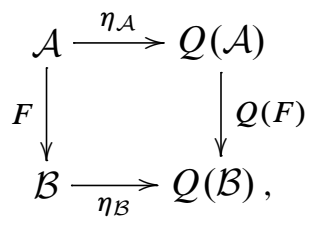

where the $\mathrm{Sp}^{\Sigma}(\mathcal{C}, K)$-functors $\eta_{\mathcal{A}}$ and $\eta_{\mathcal{B}}$ are stable quasi-equivalences by Proposition 3.40. Since the class $\mathcal{W}_{S}$ satisfies the two out of three axiom, the $\mathrm{Sp}^{\Sigma}(\mathcal{C}, K)$-functor $F$ is a stable quasi-equivalence if and only if $Q(F)$ is a stable quasi-equivalence. The $\mathrm{Sp}^{\Sigma}(\mathcal{C}, K)$-categories $Q(\mathcal{A})$ and $Q(\mathcal{B})$ satisfy condition $(\Omega)$ and so by Proposition 3.44, $Q(F)$ is a levelwise quasi-equivalence. This proves the Lemma.

Theorem 3.46 The category $\operatorname{Sp}^{\Sigma}(\mathcal{C}, K)$-Cat admits a right proper Quillen model structure whose weak equivalences are the stable quasi-equivalences (see Definition 3.32) and the cofibrations those of Theorem 3.19.

Proof The proof will consist on verifying the conditions of Theorem B.2. We consider for $\mathcal{M}$ the Quillen model structure of Theorem 3.19 and for $Q$ and $\eta$, the functor and natural transformation defined in Definition 3.37. The Quillen model structure of Theorem 3.19 is right proper (see Proposition 3.29) and by Lemma 3.45 the $Q$-weak equivalences are precisely the stable quasi-equivalences. We now verify conditions (A1), (A2) and (A3):

(A1) Let $F: \mathcal{A} \rightarrow \mathcal{B}$ be a levelwise quasi-equivalence. We have the following commutative square

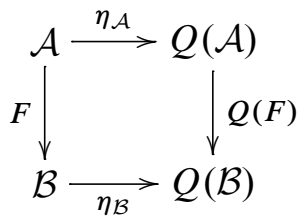

with $\eta_{\mathcal{A}}$ and $\eta_{\mathcal{B}}$ stable quasi-equivalences. Notice that since $F$ is a levelwise quasi-equivalence it is in particular a stable quasi-equivalence. The class $\mathcal{W}_{S}$ satisfies the three out of three axiom and so $Q(F)$ is a stable quasi-equivalence. Since the $Q(\mathcal{A})$ and $Q(\mathcal{B})$ satisfy condition $(\Omega)$, Proposition 3.44 implies that $Q(F)$ is in fact a levelwise quasi-equivalence. 
(A2) We now show that for every $\operatorname{Sp}^{\Sigma}(\mathcal{C}, K)$-category $\mathcal{A}$, the $\operatorname{Sp}^{\Sigma}(\mathcal{C}, K)$-functors

$$
\eta_{Q(\mathcal{A})}, Q\left(\eta_{\mathcal{A}}\right): Q(\mathcal{A}) \rightarrow Q Q(\mathcal{A})
$$

are levelwise quasi-equivalences. Since the $\operatorname{Sp}^{\Sigma}(\mathcal{C}, K)$-functors $\eta_{Q(\mathcal{A})}$ and $Q\left(\eta_{\mathcal{A}}\right)$ are stable quasi-equivalences between $\mathrm{Sp}^{\Sigma}(\mathcal{C}, K)$-categories which satisfy condition $(\Omega)$, Proposition 3.44 implies that they are levelwise quasiequivalences.

(A3) We start by observing that if $P: \mathcal{C} \rightarrow \mathcal{D}$ is a $Q$-fibration, then for all objects $x, y \in \mathcal{C}$, the morphism

$$
P(x, y): \mathcal{C}(x, y) \longrightarrow \mathcal{D}(P x, P y)
$$

is a stable fibration in $\operatorname{Sp}^{\Sigma}(\mathcal{C}, K)$, see Hovey [22, Definition 8.7]. In fact, by Lemma 3.31, the functor

$$
U: \operatorname{Sp}^{\Sigma}(\mathcal{C}, K) \longrightarrow \operatorname{Sp}^{\Sigma}(\mathcal{C}, K)-\mathrm{Cat}
$$

sends cofibrations to cofibrations. Since clearly it sends stable equivalences in $\mathrm{Sp}^{\Sigma}(\mathcal{C}, K)$ to stable quasi-equivalences in $\mathrm{Sp}^{\Sigma}(\mathcal{C}, K)$-Cat, the claim follows. Now, consider the diagram

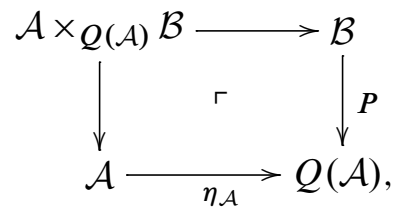

with $P$ a $Q$-fibration. The stable model structure on $\operatorname{Sp}^{\Sigma}(\mathcal{C}, K)$ is right proper by condition $(\mathrm{C} 2)$, and so we conclude, by construction of fiber products in $\operatorname{Sp}^{\Sigma}(\mathcal{C}, K)-$ Cat, that the induced $\operatorname{Sp}^{\Sigma}(\mathcal{C}, K)$-functor

$$
\eta_{\mathcal{A}_{*}}: \mathcal{A} \times{ }_{Q(\mathcal{A})} \mathcal{B} \longrightarrow \mathcal{B}
$$

satisfies condition (WE1) Since $\eta_{\mathcal{A}}$ induces the identity map on object sets so it thus $\eta_{\mathcal{A}_{*}}$. This shows condition (WE2).

\subsection{Properties II}

Proposition 3.47 $A \mathrm{Sp}^{\Sigma}(\mathcal{C}, K)$-category $\mathcal{A}$ is fibrant with respect to Theorem 3.46 if and only if for all objects $x, y \in \mathcal{A}$, the $K$-spectrum $\mathcal{A}(x, y)$ is an $\Omega$-spectrum in $\mathrm{Sp}^{\Sigma}(\mathcal{C}, K)$.

Notation 3.48 We denote these fibrant $\operatorname{Sp}^{\Sigma}(\mathcal{C}, K)$-categories by $Q$-fibrant. 
Proof By Corollary B.4, $\mathcal{A}$ is fibrant with respect to Theorem 3.46 if and only if it is fibrant for the structure of Theorem 3.19 (see Proposition 3.27) and the $\mathrm{Sp}^{\Sigma}(\mathcal{C}, K)-$ functor $\eta_{\mathcal{A}}: \mathcal{A} \rightarrow Q(\mathcal{A})$ is a levelwise quasi-equivalence. Observe that $\eta_{\mathcal{A}}$ is a levelwise quasi-equivalence if and only if for all objects $x, y \in \mathcal{A}$ the morphism

$$
\eta_{\mathcal{A}}(x, y): \mathcal{A}(x, y) \longrightarrow Q(\mathcal{A})(x, y)
$$

is a level equivalence in $\operatorname{Sp}^{\Sigma}(\mathcal{C}, K)$. Since $Q(\mathcal{A})(x, y)$ is an $\Omega$-spectrum we have the following commutative diagrams (for all $n \geq 0$ )

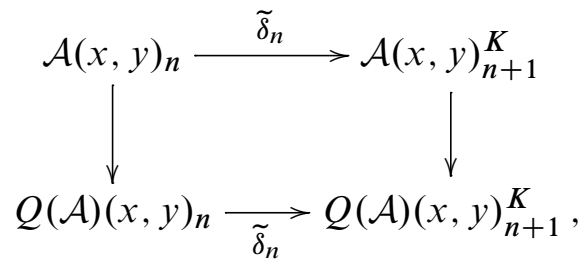

where the bottom and vertical arrows are weak equivalences in $\mathcal{C}$. This implies that

$$
\tilde{\delta}_{n}: \mathcal{A}(x, y)_{n} \longrightarrow \mathcal{A}(x, y)_{n+1}^{K}, \quad n \geq 0
$$

is a weak equivalence in $\mathcal{C}$ and so, for all $x, y \in \mathcal{A}$, the $K$-spectrum $\mathcal{A}(x, y)$ is an $\Omega$-spectrum in $\operatorname{Sp}^{\Sigma}(\mathcal{C}, K)$.

Remark 3.49 Remark 3.38 and Propositions 3.39 and 3.40 imply that $\eta_{\mathcal{A}}: \mathcal{A} \rightarrow Q(\mathcal{A})$ is a (functorial) fibrant replacement of $\mathcal{A}$ in the model structure of Theorem 3.46. In particular $\eta_{\mathcal{A}}$ induces the identity map on the set of objects. Notice also, that since the cofibrations (and so the trivial fibrations) of the model structure of Theorem 3.46 are the same as those of Theorem 3.19, Proposition 3.30 and Lemma 3.31 stay valid in the Quillen model structure of Theorem 3.46.

Proposition 3.50 Consider $F: \mathcal{A} \rightarrow \mathcal{B}$, a $\operatorname{Sp}^{\Sigma}(\mathcal{C}, K)$-functor between $Q$-fibrant $\mathrm{Sp}^{\Sigma}(\mathcal{C}, K)$-categories. Then $F$ is a $Q$-fibration if and only if it is a fibration in the model structure of Theorem 3.19 (see Proposition 3.26).

Proof By Theorem B.3, if $F$ is a $Q$-fibration then it is a fibration. Let us now prove the converse. Suppose that $F: \mathcal{A} \rightarrow \mathcal{B}$ is a fibration. Consider the commutative square

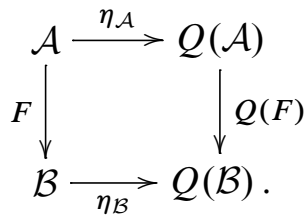


Since $\mathcal{A}$ and $\mathcal{B}$ satisfy the condition $(\Omega)$, Proposition 3.44 implies that $\eta_{\mathcal{A}}$ and $\eta_{\mathcal{B}}$ are levelwise quasi-equivalences. Since the model structure of Theorem 3.19 is right proper (see Proposition 3.29), the previous square is homotopy cartesian and so by Theorem B.3, $F$ is in fact a $Q$-fibration.

Remark 3.51 Since a $Q$-fibration is a fibration, the adjunction of Remark 3.28 induces a natural Quillen adjunction

$$
\begin{gathered}
\operatorname{Sp}^{\Sigma}(\mathcal{C}, K)-\mathrm{Cat} \\
\left.F_{0} \uparrow\right|_{\mathcal{C}-\mathrm{Cat}} ^{\mathrm{Ev}_{0}}
\end{gathered}
$$

with respect to the model structure of Theorem 3.46.

\subsection{Homotopy Idempotence}

In [22, Theorem 9.1], Hovey proved the following idempotence property: if the functor $-\otimes K: \mathcal{C} \longrightarrow \mathcal{C}$ is a Quillen equivalence, then the Quillen adjunction

$$
\begin{gathered}
\operatorname{Sp}^{\Sigma}(\mathcal{C}, K) \\
\left.F_{0}\right|_{\mathcal{C}} \downarrow^{\mathrm{Ev}_{0}} \\
\end{gathered}
$$

is a Quillen equivalence, where $\operatorname{Sp}^{\Sigma}(\mathcal{C}, K)$ is endowed with the stable model structure.

Theorem 3.52 If the functor $-\otimes K: \mathcal{C} \longrightarrow \mathcal{C}$ is a Quillen equivalence, then the Quillen adjunction of Remark 3.51

$$
\begin{gathered}
\operatorname{Sp}^{\Sigma}(\mathcal{C}, K)-C a t \\
\left.F_{0} \uparrow\right|^{\mathrm{Ev}_{0}} \\
\mathcal{C}-C a t
\end{gathered}
$$

is a Quillen equivalence.

Proof The proof will consist on verifying conditions (a) and (b) of Proposition 2.14.

(a) Let $F: \mathcal{A} \rightarrow \mathcal{B}$ be a $\operatorname{Sp}^{\Sigma}(\mathcal{C}, K)$-functor between $Q$-fibrant $\operatorname{Sp}^{\Sigma}(\mathcal{C}, K)$-categories, such that $\operatorname{Ev}_{0}(\mathcal{A}): \operatorname{Ev}_{0}(\mathcal{A}) \rightarrow \operatorname{Ev}_{0}(\mathcal{B})$ is a weak equivalence in $\mathcal{C}-\mathrm{Cat}$. 
Since $\mathcal{A}$ and $\mathcal{B}$ satisfy condition $(\Omega)$, condition (a) of Proposition 2.14 applied to the Quillen equivalence

$$
\mathrm{Ev}_{0}: \operatorname{Sp}^{\Sigma}(\mathcal{C}, K) \longrightarrow \mathcal{C}
$$

implies that $F$ satisfies condition (WE1) Notice also that since $\operatorname{Ev}_{0}(F)$ is a weak equivalence in $\mathcal{C}$-Cat, Corollary 3.43 implies that $F$ satisfies condition (WE2). In conclusion $F$ is a stable quasi-equivalence.

(b) Let $\mathcal{A}$ be a cofibrant $\mathcal{C}$-category and let us denote by $I$ its set of objects. Notice, that in particular $\mathcal{A}$ is cofibrant in $\mathcal{C}^{I}$-Cat, with respect to the model structure of Remark A.2. Now, since the adjunction

$$
\begin{gathered}
\operatorname{Sp}^{\Sigma}(\mathcal{C}, K) \\
\left.\left.F_{0}\right|_{\mathcal{C}}\right|^{\mathrm{Ev}_{0}} \\
\end{gathered}
$$

is a strong monoidal Quillen equivalence (see Definition 2.5), Remark A.3 implies that condition (b) of Proposition 2.14 is verified for the adjunction $\left(F_{0}^{I}, \operatorname{Ev}_{0}^{I}\right)$. By Remark 3.49, the $\operatorname{Sp}^{\Sigma}(\mathcal{C}, K)$-functor $\eta_{\mathcal{A}}: \mathcal{A} \rightarrow Q(\mathcal{A})$ is a fibrant resolution in $\operatorname{Sp}^{\Sigma}(\mathcal{C}, K)^{I}$ and so we conclude that condition (b) is verified.

Remark 3.53 Clearly Example (1) of Example 3.1 satisfies Hovey's condition, since the functor $-\otimes \mathbb{Z}[1]: \mathbf{C h} \rightarrow \mathbf{C h}$ is the classical suspension functor. This implies, by Theorem 3.52, that we have a Quillen equivalence

$$
\begin{gathered}
\mathrm{Sp}^{\Sigma}(\mathbf{C h}, \mathbb{Z}[1])-\mathrm{Cat} \\
\left.F_{0} \uparrow\right|_{\text {dgcat }} \mathrm{Ev}_{0}
\end{gathered}
$$

\section{A Quillen equivalence criterion}

Let $\mathcal{C}$ and $\mathcal{D}$ be two symmetric monoidal model categories, with $K$ an object of $\mathcal{C}$ and $K^{\prime}$ an object of $\mathcal{D}$. In this Section we establish a general criterion for when a Quillen equivalence between $\operatorname{Sp}^{\Sigma}(\mathcal{C}, K)$ and $\operatorname{Sp}^{\Sigma}\left(\mathcal{D}, K^{\prime}\right)$ induces a Quillen equivalence between $\mathrm{Sp}^{\Sigma}(\mathcal{C}, K)-$ Cat and $\mathrm{Sp}^{\Sigma}\left(\mathcal{D}, K^{\prime}\right)$-Cat, see Theorem 4.2.

Assume that $\mathcal{C}, K, \mathcal{C}-$ Cat and $\mathcal{D}, K^{\prime}, \mathcal{D}$-Cat satisfy conditions $(\mathrm{C} 1)-(\mathrm{C} 6)$ and condition $(\mathrm{H})$ of the previous Section. Assume also the existence of a weak monoidal 
Quillen equivalence (see Definition 2.5)

$$
\begin{gathered}
\operatorname{Sp}^{\Sigma}(\mathcal{C}, K) \\
L \uparrow \downarrow^{\uparrow} \\
\operatorname{Sp}^{\Sigma}\left(\mathcal{D}, K^{\prime}\right),
\end{gathered}
$$

between the stable Quillen model structures (see Hovey [22, Definition 8.7]), which preserves level fibrations and level equivalences. By Proposition A.4, we obtain the following adjunction

$$
\begin{gathered}
\operatorname{Sp}^{\Sigma}(\mathcal{C}, K)-\mathrm{Cat} \\
L_{\text {cat }} \uparrow \downarrow^{N} \\
\operatorname{Sp}^{\Sigma}\left(\mathcal{D}, K^{\prime}\right)-\text { Cat } .
\end{gathered}
$$

Remark 4.1 The functor $N$ restricts to a functor $\tilde{N}$ :

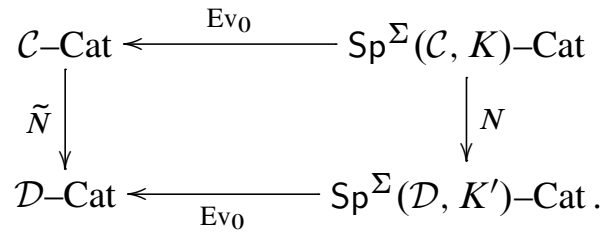

The specific nature of the model structures on the right hand-side categories (see Theorem 3.46), combined with the general Theorems 2.13 and 2.14, allow us to reduce properties of the adjunction $\left(L_{\text {cat }}, N\right)$ to simple properties of the functor $\tilde{N}$ (which are verified in all our examples, see Section 4.1). This motivated the formulation of the following abstract Quillen equivalence criterion.

Theorem 4.2 If there exists a functor

$$
\tilde{N}: \mathcal{C}-\text { Cat } \longrightarrow \mathcal{D}-\text { Cat }
$$

which preserves fibrations and weak equivalences and makes the following diagram

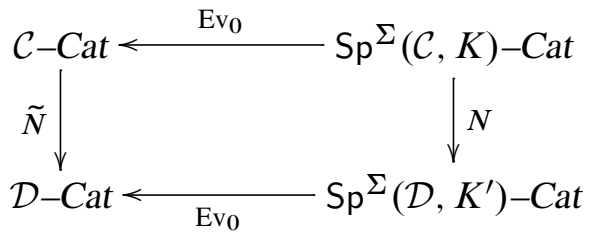

commute, then the adjunction ( $\left.L_{\text {cat }}, N\right)$ is a Quillen adjunction (with respect to the model structure of Theorem 3.46). Moreover, if $\tilde{N}$ satisfies the following condition: 
(R) Let $F: \mathcal{A} \rightarrow \mathcal{B}$ be a $\mathcal{C}$-functor. If the functor $[\tilde{N}(F)]$ (see Remark 2.7) is essentially surjective so it is the functor $[F]$,

then the Quillen adjunction $\left(L_{\text {cat }}, N\right)$ is a Quillen equivalence.

Proof We start by showing that $\left(L_{\text {cat }}, N\right)$ is a Quillen adjunction with respect to the model structure of Theorem 3.19. Observe that since the functor $N: \mathrm{Sp}^{\Sigma}(\mathcal{C}, K) \longrightarrow$ $\mathrm{Sp}^{\Sigma}\left(\mathcal{D}, K^{\prime}\right)$ preserves fibrations and levelwise weak equivalences, Proposition 3.26, Definition 3.14 and the above commutative square imply that

$$
N: \operatorname{Sp}^{\Sigma}(\mathcal{C}, K)-\mathrm{Cat} \longrightarrow \mathrm{Sp}^{\Sigma}\left(\mathcal{D}, K^{\prime}\right)-\mathrm{Cat}
$$

preserves fibrations and levelwise quasi-equivalences. We now show that $\left(L_{\text {cat }}, N\right)$ induces a Quillen adjunction with respect to the model structure of Theorem 3.46. For this, we use Proposition 2.13. The functor $N$ clearly preserves trivial fibrations since these are the same in both model structures. Let $F: \mathcal{A} \rightarrow \mathcal{B}$ be a $Q$-fibration between Q-fibrant objects (see Proposition 3.47). By Proposition 3.50, $F$ is a fibration and so it is $N(F)$. Since condition $(\Omega)$ is preserved by the functor $N$, Proposition 3.50 implies that the $\operatorname{Sp}^{\Sigma}\left(\mathcal{D}, K^{\prime}\right)$-functor $N(F)$ is in fact a $Q$-fibration.

We now show that if condition $(\mathrm{R})$ is satisfied, the adjunction $\left(L_{\text {cat }}, N\right)$ is a Quillen equivalence. For this, we verify conditions (a) and (b) of Proposition 2.14.

(a) Let $F: \mathcal{A} \rightarrow \mathcal{B}$ be a $\operatorname{Sp}^{\Sigma}(\mathcal{C}, K)$-functor between $Q$-fibrant objects, such that $N(F)$ is a stable quasi-equivalence in $\operatorname{Sp}^{\Sigma}\left(\mathcal{D}, K^{\prime}\right)$-Cat. Since condition $(\Omega)$ is preserved by the functor $N$, Proposition 3.44 implies that $N(F)$ is a levelwise quasi-equivalence. Moreover, since $\mathcal{A}$ and $\mathcal{B}$ are $Q$-fibrant, condition (a) of Proposition 2.14 applied to the Quillen equivalence

$$
N: \operatorname{Sp}^{\Sigma}(\mathcal{C}, K) \longrightarrow \operatorname{Sp}^{\Sigma}\left(\mathcal{D}, K^{\prime}\right)
$$

implies that $F$ satisfies condition (WE1) By condition (R) and the previous commutative square $F$ satisfies also condition (L2'). Finally by Proposition 3.44 , we conclude that $F$ is a stable quasi-equivalence.

(b) Let $\mathcal{A}$ be a cofibrant $\mathrm{Sp}^{\Sigma}\left(\mathcal{D}, K^{\prime}\right)$-category and denote by $I$ its set of objects. Notice that $\mathcal{A}$ is also cofibrant in $\operatorname{Sp}^{\Sigma}\left(\mathcal{D}, K^{\prime}\right)^{I}$-Cat (with respect to the module structure of Remark A.2). Since the adjunction $(L, N)$ is a strong monoidal Quillen equivalence (see Definition 2.5), Remark A.3 implies that condition (b) of Proposition 2.14 is verified by the adjunction $\left(L^{I}, N^{I}\right)$. Finally Remark 3.49 implies that the $\operatorname{Sp}^{\Sigma}(\mathcal{C}, K)$-functor $\eta_{\mathcal{A}}: \mathcal{A} \rightarrow Q(\mathcal{A})$ is a fibrant resolution of $\mathcal{A}$ in $\operatorname{Sp}^{\Sigma}(\mathcal{C}, K)^{I}$ which allow us to conclude that the condition (b) is verified. $\square$ 


\subsection{Examples of Quillen equivalences}

In this Subsection we describe two examples of Quillen equivalences which satisfy the conditions of Theorem 4.2.

4.1.1 First example We describe the relationship between Examples (1) and (2) of model structures described in Example 3.1 and Example 3.10. Thanks to Shipley [32, Proposition 4.9] the inclusion functor $i: \mathbf{C h}_{\geq 0} \rightarrow \mathbf{C h}$ induces a strong monoidal Quillen equivalence

$$
\begin{gathered}
\operatorname{Sp}^{\Sigma}(\mathbf{C h}, \mathbb{Z}[1]) \\
i \uparrow \mid \tau_{\geq 0} \\
\operatorname{Sp}^{\Sigma}\left(\mathbf{C h}_{\geq 0}, \mathbb{Z}[1]\right),
\end{gathered}
$$

with respect to the stable model structures. Moreover, the truncation functor $\tau_{\geq 0}$ preserves fibrations and level equivalences. By Remark A.5, we obtain an adjunction

$$
\begin{gathered}
\operatorname{Sp}^{\Sigma}(\mathbf{C h}, \mathbb{Z}[1])-\mathrm{Cat} \\
i \uparrow \mid \tau_{\geq 0} \\
\operatorname{Sp}^{\Sigma}\left(\mathbf{C h}_{\geq 0}, \mathbb{Z}[1]\right)-\text { Cat } .
\end{gathered}
$$

Notice that we have the following commutative square

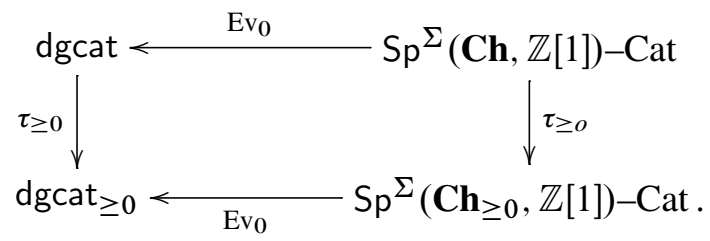

Finally, since the functor

$$
\tau_{\geq 0}: \text { dgcat } \longrightarrow \text { dgcat }_{\geq 0}
$$

clearly satisfies condition $(\mathrm{R})$ of Theorem 4.2, the previous adjunction is a Quillen equivalence.

4.1.2 Second example We describe the relationship between Examples (2) and (3) of model structures described in Example 3.1 and Example 3.10. Thanks to Shipley [32, 
Proposition 4.4] we have a weak monoidal Quillen equivalence

$$
\begin{gathered}
\operatorname{Sp}^{\Sigma}\left(S \mathbf{A b}, \widetilde{\mathbb{Z}}\left(S^{1}\right)\right) \\
L \uparrow \mid \phi^{*} N \\
\operatorname{Sp}^{\Sigma}\left(\mathbf{C h}_{\geq 0}, \mathbb{Z}[1]\right),
\end{gathered}
$$

with respect to the stable model structures. Moreover the functor $\phi^{*} N$ preserves fibrations and levelwise weak equivalences (see Shipley's proof [32, Proposition 4.4]). By Proposition A.4, we obtain an adjunction

$$
\begin{aligned}
\operatorname{Sp}^{\Sigma}\left(s \mathbf{A b}, \widetilde{\mathbb{Z}}\left(S^{1}\right)\right)-\mathrm{Cat} \\
L_{\text {cat }} \uparrow \mid \phi^{*} N \\
\operatorname{Sp}^{\Sigma}\left(\mathbf{C h}_{\geq 0}, \mathbb{Z}[1]\right)-\text { Cat } .
\end{aligned}
$$

Recall from Shipley [32, Proposition 4.4] that the ring map

$$
\phi: \operatorname{Sym}(\mathbb{Z}[1]) \longrightarrow \mathcal{N}
$$

is the identity in degree zero. This implies that we have the following commutative square

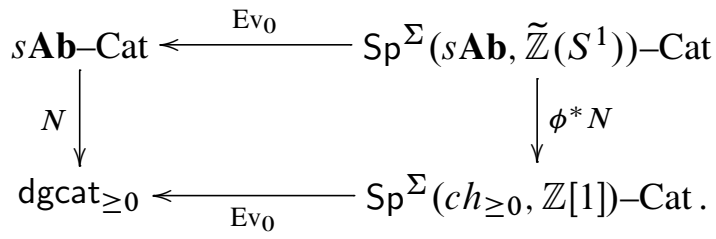

Finally, since the normalization functor (see Tabuada [38, Section 5.2])

$$
N: s \mathbf{A b}-\mathrm{Cat} \longrightarrow \text { dgcat }_{\geq 0}
$$

satisfies condition (R) of Theorem 4.2, the adjunction $\left(L_{\mathrm{cat}}, \phi^{*} N\right)$ is a Quillen equivalence.

\section{General spectral algebra}

Let $R$ be a commutative symmetric ring spectrum of pointed simplicial sets, see Schwede [28, Definition 1.3-I]. In this Section, we construct a Quillen model structure on the category of small categories enriched over $R$-modules, see Theorem 5.17. Since the arguments used in this Section are conceptually very similar to those of Section 3, we have deferred all the proofs to Appendix D. 


\subsection{Classical results of spectral algebra}

Let us denote by $\left(R-\operatorname{Mod},-\wedge_{R}-, R\right)$ the symmetric monoidal category of right $R$-modules, see Schwede [28, Definition 1.4-I].

Theorem 5.1 (see Schwede [28, Theorem 3.2-III]) The category $R-M o d$ carries the following model structures:

- The projective level model structure, in which the weak equivalences (resp. fibrations) are those morphisms of $R$-modules which are projective level equivalences (resp. projective level fibrations) on the underlying symmetric spectra.

- The projective stable model structure, in which the weak equivalences (resp. fibrations) are those morphisms of $R$-modules which are projective stable equivalences (resp. projective stable fibrations) on the underlying symmetric spectra.

Moreover these model structures are proper, simplicial, cofibrantly generated, monoidal (with respect to the smash product over $R$ ) and satisfy the monoid axiom.

Let $f: P \rightarrow R$ be a morphism of commutative symmetric ring spectra, see [28, Definition 1.3-I]. We have a restriction/extension of scalars adjunction

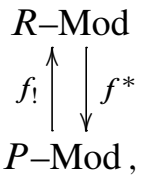

with $f$ ! a strong monoidal functor and $f^{*}$ a lax monoidal one. Given a $R$-module $M$, we define the $P$-module $f^{*}(M)$ as having the same underlying symmetric spectrum as $M$ and whose $P$-action is given by the composition

$$
\left(f^{*} M\right) \wedge P=M \wedge P \stackrel{\mathrm{Id} \wedge f}{\longrightarrow} M \wedge R \stackrel{\alpha}{\longrightarrow} M,
$$

where $\alpha$ denotes the $R$-action on $M$. The left adjoint functor $f_{!}$sends a $P$-module $N$ to the $R$-module $N \wedge_{P} R$ whose $R$-action is given by

$$
f_{!} N \wedge R=N \wedge_{P} R \wedge R \stackrel{\mathrm{Id} \wedge \mu}{\longrightarrow} N \wedge_{P} R
$$

where $\mu$ denotes the multiplication of $R$.

Theorem 5.2 (see Schwede [28, Theorem 3.4-III]) The previous adjunction $\left(f_{!}, f^{*}\right)$ is a Quillen adjunction with respect to both model structures of Theorem 5.1. 
We now focus in the case where $P$ is the sphere symmetric ring spectrum $\mathbb{S}$ (see Schwede [28, Example 2.1-I]) and $f=i$ the unique morphism of ring spectra. We have a restriction/extension of scalars adjunction

$$
\begin{gathered}
R-\mathrm{Mod} \\
i ! \mid i^{*} \\
\mathrm{Sp}^{\Sigma},
\end{gathered}
$$

where $\mathrm{Sp}^{\Sigma}$ denotes the category of symmetric spectra (see Hovey-Shipley-Smith [23]).

Notation 5.3 From now on and until the end of the Section, we denote (to simplify the notation) by $R$-Cat the category ( $R-$ Mod)-Cat (see Notation 2.2$)$. In particular a $(R-$ Mod $)-$ category will be denoted a $R$-category and a ( $R$-Mod)-functor a $R-$ functor.

By Remark A.5, the previous adjunction $\left(i_{!}, i^{*}\right)$ induces the following one

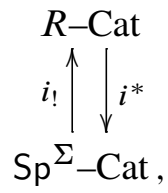

where $\mathrm{Sp}^{\Sigma}$-Cat denotes the category of spectral categories, see Tabuada [37].

\subsection{Quillen model structure}

Recall that the model structure on $\mathrm{Sp}^{\Sigma}$-Cat described by Tabuada in [37, Theorem 5.10] (or equivalently the one of Theorem 3.46 corresponding to Example (0) in Example 3.1 and Example 3.10) is constructed from the levelwise model structure using the Bousfield localization techniques of Appendix B. To the best of the author's knowledge, it is not known if these localization techniques preserve the cofibrant generated nature of model structures. Moreover, it is not clear how to describe generic pushouts in $R-$ Cat. Therefore the Lifting Theorem (see Hirschhorn [20, Theorem 11.3.2]) can not be directly applied to the previous adjunction. We sidestep this difficulty as follows:

- First, we lift the cofibrantly generated model structure on $\mathrm{Sp}^{\Sigma}$-Cat (whose weak equivalences are the levelwise quasi-equivalences) along the previous adjunction (see Theorem 5.5) and obtain a model structure on $R$-Cat whose weak equivalences are the levelwise quasi-equivalences, see Definition 5.4. 
- Then, we lift along the previous adjunction the spectral functors used in the construction of a localization functor in $S p^{\Sigma}-C a t$, and use them to define a functor (see Section 5.3)

$$
Q: R-\text { Cat } \longrightarrow R-\text { Cat }
$$

By localizing the levelwise model structure with respect to this functor, we obtain finally a model structure on $R$-Cat (see Theorem 5.17), whose weak equivalences are the stable quasi-equivalences, see Definition 5.8.

Although the model structure on $R$-Cat of Theorem 5.17 is not obtained by lifting the model structure on $\mathrm{Sp}^{\Sigma}$-Cat along the previous adjunction, Propositions 5.11 and 5.22 imply that its weak equivalences and fibrations (and so the entire model structure) are determined by the restriction functor $i^{*}$.

\subsubsection{Levelwise quasi-equivalences}

Definition 5.4 A $R$-functor $F: \mathcal{A} \rightarrow \mathcal{B}$ is a levelwise quasi-equivalence, resp. a fibration, if the restricted spectral functor $i^{*}(F): i^{*}(\mathcal{A}) \rightarrow i^{*}(\mathcal{B})$ is a levelwise quasiequivalence (see Definition 3.14), resp. a fibration (see Proposition 3.26) in $\mathrm{Sp}^{\Sigma}$-Cat.

Theorem 5.5 If we let $\mathcal{M}$ be the category $\mathrm{Sp}^{\Sigma}$-Cat, endowed with the model structure described by Tabuada in [37, Theorem 4.8], and $\mathcal{N}$ be the category $R-C a t$, then the conditions of the lifting Theorem (see Hirschhorn [20, Theorem 11.3.2]) are satisfied. Thus, the category $R-C a t$ admits a cofibrantly generated Quillen model structure whose weak equivalences are the levelwise quasi-equivalences.

Remark 5.6 Since the Quillen model structure on $\mathrm{Sp}^{\Sigma}$-Cat is right proper (see Proposition 3.29), the same holds for the model structure on $R$-Cat of Theorem 5.5. Moreover, by Proposition 3.27, a $R$-category $\mathcal{A}$ is fibrant if and only if for all objects $x, y \in \mathcal{A}$ and $n \geq 0$, the pointed simplicial set $i^{*}(\mathcal{A})(x, y)_{n}$ is fibrant.

Proposition 5.7 Let $\mathcal{A}$ be a cofibrant $R$-category. Then for all objects $x, y \in \mathcal{A}$, the $R$-module $\mathcal{A}(x, y)$ is cofibrant in the projective level model structure on $R-M o d$ (see Theorem 5.1).

\subsubsection{Stable quasi-equivalences}

Definition 5.8 A $R$-functor $F: \mathcal{A} \rightarrow \mathcal{B}$ is a stable quasi-equivalence if: 
- for all objects $x, y \in \mathcal{A}$, the morphism

$$
F(x, y): \mathcal{A}(x, y) \longrightarrow \mathcal{B}(F x, F y)
$$

is a stable equivalence in $R-$ Mod and

- the induced functor (see Remark 2.7)

$$
[F]:[\mathcal{A}] \longrightarrow[\mathcal{B}]
$$

is an equivalence of categories.

Remark 5.9 Notice that Definition 5.8 is a particular case of the general Definition 2.8 , where we have taken $R$-Mod for the base symmetric monoidal model category.

Notation 5.10 We denote by $\mathcal{W}_{S}$ the class of stable quasi-equivalences.

Proposition 5.11 A $R$-functor $F: \mathcal{A} \rightarrow \mathcal{B}$ is a stable quasi-equivalence if and only if the restricted spectral functor $i^{*}(F): i^{*}(\mathcal{A}) \rightarrow i^{*}(\mathcal{B})$ is a stable quasi-equivalence in $\mathrm{Sp}^{\Sigma}-$ Cat (see Definition 3.32).

\section{$5.3 Q$-functor}

We now construct a functor

$$
Q: R-\mathrm{Cat} \longrightarrow R-\mathrm{Cat}
$$

and a natural transformation $\eta: \mathrm{Id} \rightarrow Q$, from the identity functor on $R-$ Cat to $Q$. Recall from Tabuada [37, Section 5.1] that we have at our disposal a specific set $\left\{A_{m, k, n}\right\} \cup U(K)$ of spectral functors used in the construction of a localization functor in $\mathrm{Sp}^{\Sigma}$-Cat.

Definition 5.12 Let $\mathcal{A}$ be a $R$-category. The functor $Q: R-$ Cat $\longrightarrow R-$ Cat is obtained by applying the small object argument, using the set $\left\{i_{!}\left(A_{m, k, n}\right)\right\} \cup i_{!} U(K)$, to factorize the $R$-functor $\mathcal{A} \longrightarrow \bullet$, where $\bullet$ denotes the terminal object in $R$-Cat.

Remark 5.13 We obtain in this way a functor $Q$ and a natural transformation $\eta: \mathrm{Id} \rightarrow Q$. Notice that the set $\left\{i_{!}\left(A_{m, k, n}\right)\right\} \cup i_{!} U(K)$ identifies naturally with the set $\left\{U\left(i_{!}\left(A_{m, n, k}\right)\right)\right\} \cup U\left(i_{!}(K)\right)$. Notice also that $Q(\mathcal{A})$ has the same objects as $\mathcal{A}$, and the RLP with respect to the set $\left\{U\left(i_{!}\left(A_{m, n, k}\right)\right)\right\} \cup U\left(i_{!}(K)\right)$. Thanks to Tabuada [37, Remark 5.7] this is equivalent to the spectral category $i^{*} Q(\mathcal{A})$ satisfying the following condition. 
$(\Omega)$ for all objects $x, y \in i^{*} Q(\mathcal{A})$ the symmetric spectra $\left(i^{*} Q\right)(x, y)$ is an $\Omega$ spectrum.

Proposition 5.14 Let $\mathcal{A}$ be a $R$-category. The $R$-functor

$$
\eta_{\mathcal{A}}: \mathcal{A} \longrightarrow Q(\mathcal{A})
$$

is a stable quasi-equivalence (see Definition 5.8).

\subsection{Main Theorem}

Definition 5.15 A $R$-functor $F: \mathcal{A} \rightarrow \mathcal{B}$ is

- a $Q$-weak equivalence if $Q(F)$ is a levelwise quasi-equivalence (see Definition 5.4).

- a cofibration if it is a cofibration in the model structure of Theorem 5.5 .

- a $Q$-fibration if it has the RLP with respect to all cofibrations which are $Q$-weak equivalences.

Lemma 5.16 A $R$-functor $F: \mathcal{A} \rightarrow \mathcal{B}$ is a $Q$-weak equivalence if and only if it is a stable quasi-equivalence.

Theorem 5.17 The category $R-C a t$ admits a right proper Quillen model structure whose weak equivalences are the stable quasi-equivalences (see Definition 5.8) and the cofibrations are those of Theorem 5.5.

Proposition 5.18 A $R$-category $\mathcal{A}$ is fibrant with respect to the model structure of Theorem 5.17 if and only if for all objects $x, y \in \mathcal{A}$, the symmetric spectrum $\left(i^{*} \mathcal{A}\right)(x, y)$ is an $\Omega$-spectrum.

Notation 5.19 We denote these fibrant $R$-categories by $Q$-fibrant.

Remark 5.20 Remark 5.13 and Proposition 5.18 imply that $\eta_{\mathcal{A}}: \mathcal{A} \rightarrow Q(\mathcal{A})$ is a (functorial) fibrant replacement of $\mathcal{A}$ in the model structure of Theorem 5.17. In particular $\eta_{\mathcal{A}}$ induces the identity map on the set of objects. Notice also, that the cofibrations (and so the trivial fibrations) of the model structure of Theorem 5.17 coincide with those of the model structure of Theorem 5.5. In particular a $R$-category is cofibrant in the model structure of Theorem 5.17 if and only if it is cofibrant in the model structure of Theorem 5.5, see Proposition 5.7. 
Let $f: P \rightarrow R$ be a morphism of commutative symmetric ring spectra. The restriction/extension of scalars adjunction induces a natural adjunction

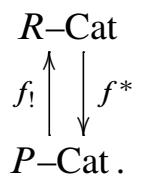

Proposition 5.21 The previous adjunction $\left(f_{!}, f^{*}\right)$ is a Quillen adjunction with respect to the model structure of Theorem 5.5 and of Theorem 5.17.

We finish this Section by giving a simple characterization of the $Q$-fibrations (see Definition 5.15) in $R-$ Cat in terms of the restriction functor $i^{*}$.

Proposition 5.22 A $R$-functor $F: \mathcal{A} \rightarrow \mathcal{B}$ is a $Q$-fibration if and only if the restricted spectral functor $i^{*}: i^{*}(\mathcal{A}) \rightarrow i^{*}(\mathcal{B})$ is a $Q$-fibration in $\mathrm{Sp}^{\Sigma}$-Cat (see Definition 3.41).

\section{Eilenberg-Mac Lane spectral algebra}

In this Section we prove that when the commutative symmetric ring spectrum $R$ is the Eilenberg-Mac Lane symmetric ring spectrum $H \mathbb{Z}$, the model structure of Theorem 5.17 is Quillen equivalent to the one of Theorem 3.46 (with $\mathcal{C}=s \mathbf{A b}$ and $K=\widetilde{\mathbb{Z}}\left(S^{1}\right)$ ), see Proposition 6.1. Recall from Shipley [32, Proposition 4.3] that we have the following strong monoidal Quillen equivalence

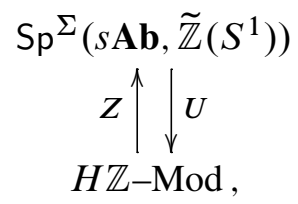

with $U$ the forgetful functor. By Remark A.5, we obtain the following adjunction

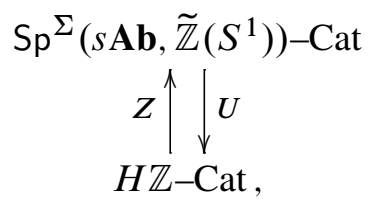

which we denoted by the same functors.

Proposition 6.1 The previous adjunction is a Quillen equivalence, when the category $\mathrm{Sp}^{\Sigma}\left(s \mathbf{A b}, \widetilde{\mathbb{Z}}\left(S^{1}\right)\right)-C a t$ is endowed with the model structure of Theorem 3.46 and $H \mathbb{Z}-C a t$ is endowed with the model structure of Theorem 5.17. 
Proof We start by showing that the previous adjunction is a Quillen adjunction, when $\mathrm{Sp}^{\Sigma}(s \mathbf{A b})-$ Cat is endowed with the model structure of Theorem 3.19 and $H \mathbb{Z}$-Cat is endowed with the model structure of Theorem 5.5. Notice that we have the following commutative diagram

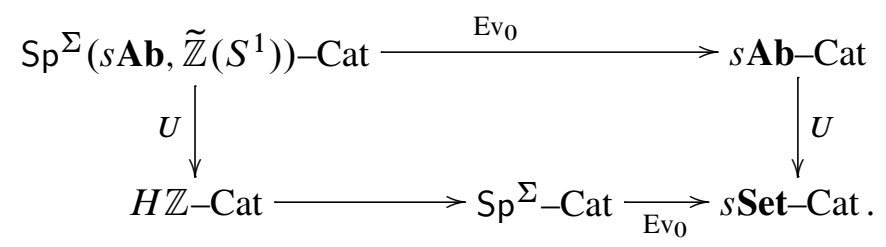

Since the forgetful functor

$$
U: \operatorname{Sp}^{\Sigma}\left(s \mathbf{A b}, \widetilde{\mathbb{Z}}\left(S^{1}\right)\right) \rightarrow H \mathbb{Z}-\mathrm{Cat}
$$

preserves fibrations and levelwise equivalences, the claim follows from the previous diagram. Let us now show that it is a Quillen adjunction with respect to the model structures of the Proposition. Since by Lemmas D.4 and 3.50, fibrations between $Q$-fibrant objects are $Q$-fibrations the functor $U$ preserves them. Clearly the functor $U$ preserves also the trivial fibrations. This implies, by Proposition 2.13 that the adjunction is a Quillen adjunction.

Now, we show that it is a Quillen equivalence. For this, we verify conditions (a) and (b) of Proposition 2.14.

(a) Let $R: \mathcal{A} \rightarrow \mathcal{B}$ be a $\operatorname{Sp}^{\Sigma}\left(s \mathbf{A b}, \widetilde{\mathbb{Z}}\left(S^{1}\right)\right)$-functor between $Q$-fibrant objects such that $U(R): U(\mathcal{A}) \rightarrow U(\mathcal{B})$ is a stable quasi-equivalence. Since $\mathcal{A}$ and $\mathcal{B}$ are $Q$-fibrant, condition (a) of Proposition 2.14 applied to the Quillen equivalence

$$
U: \operatorname{Sp}^{\Sigma}\left(s \mathbf{A b}, \widetilde{\mathbb{Z}}\left(S^{1}\right)\right) \longrightarrow H \mathbb{Z}-\mathrm{Mod}
$$

implies that $R$ satisfies condition (WE1) Moreover, since the functor

$$
U: s \mathbf{A b}-\mathrm{Cat} \rightarrow s \text { Set-Cat }
$$

satisfies condition (R) of Theorem 4.2, the $\mathrm{Sp}^{\Sigma}\left(s \mathbf{A b}, \widetilde{\mathbb{Z}}\left(S^{1}\right)\right)$-functor $R$ satisfies also condition (L2'), which allow us to conclude that it is a stable quasiequivalence.

(b) Let $\mathcal{A}$ be a cofibrant $H \mathbb{Z}$-category and let us denote by $I$ its set of objects. Notice that $\mathcal{A}$ is cofibrant in $(H \mathbb{Z}-\mathrm{Mod})^{I}-$ Cat (with respect to the model structure of Remark A.2). Since the adjunction $(L, F)$ is a strong monoidal Quillen equivalence (see Definition 2.5), Remark 5.20 implies that condition (b) of Proposition 2.14 is verified by the adjunction $\left(L^{I}, F^{I}\right)$. By Remark 
A.3, the $\operatorname{Sp}^{\Sigma}(\mathcal{C}, K)$-functor $\eta_{\mathcal{A}}: \mathcal{A} \rightarrow Q(\mathcal{A})$ is a fibrant resolution of $\mathcal{A}$ in $(H \mathbb{Z}-\mathrm{Mod})^{I}-\mathrm{Cat}$ and so we conclude that condition (b) is verified.

\section{Global picture}

By Remarks 3.53, 4.1.1, 4.1.2 and Proposition 6.1 we have the following (four steps) zig-zag of Quillen equivalences:

$$
\begin{aligned}
& H \mathbb{Z} \text {-Cat } \\
& Z \downarrow \uparrow U \\
& \mathrm{Sp}^{\Sigma}\left(s \mathbf{A b}, \widetilde{\mathbb{Z}}\left(S^{1}\right)\right)-\mathrm{Cat} \\
& L_{\text {cat }} \uparrow \downarrow \phi^{*} N \\
& \mathrm{Sp}^{\Sigma}\left(\mathbf{C h}_{\geq 0}, \mathbb{Z}[1]\right)-\mathrm{Cat} \\
& i \downarrow \uparrow \tau_{\geq 0} \\
& \mathrm{Sp}^{\Sigma}(\mathbf{C h}, \mathbb{Z}[1])-\mathrm{Cat} \\
& F_{0} \uparrow \downarrow \mathrm{Ev}_{0} \\
& \text { dgcat . }
\end{aligned}
$$

Notation 7.1 Let us denote by

$$
\mathbb{H}: \mathrm{Ho}(\text { dgcat }) \stackrel{\sim}{\longrightarrow} \mathrm{Ho}(H \mathbb{Z}-\mathrm{Cat})
$$

and by

$$
\Theta: \mathrm{Ho}(H \mathbb{Z}-\mathrm{Cat}) \stackrel{\sim}{\longrightarrow} \mathrm{Ho}(\mathrm{dgcat})
$$

the composed derived equivalences.

Remark 7.2 If, in the previous zig-zag, we restrict ourselves to enriched categories with a single object, we recover the zig-zag of Quillen equivalences constructed by Shipley in [32, Propositions 2.10 and 4.9].

\section{Applications}

In this Section we describe two applications of the zig-zag of Quillen equivalences of the previous Section. Our first application is a conceptual proof of the invariance under quasi-equivalences (see Example 2.10) of the topological Hochschild homology 
(THH) and topological cyclic homology (TC) of dg categories. Our second application concerns the construction of trace maps from algebraic $K$-theory to topological Hochschild homology.

\subsection{THH and TC}

Definition 8.1 (Blumberg-Mandell's construction) Building upon the work of McCarthy and Dundas [11] (which, in turn, was based on Bökstedt's original construction [3]), Blumberg and Mandell started by defining THH and TC for spectral categories, see [2, Section 3]. Let $\mathcal{I}$ be the category whose objects are the finite sets $\mathbf{n}=\{1, \ldots, n\}$ (including $\mathbf{0}=\{\}$ ), and whose morphisms are the injective maps. For a symmetric spectrum $T$ (of pointed simplicial sets), we write $T_{n}$ for the $n$th pointed simplicial set. The association $\mathbf{n} \mapsto \Omega^{n}\left|T_{n}\right|$ extends to a functor from $\mathcal{I}$ to spaces, where $|-|$ denotes the geometric realization.

More generally, given symmetric spectra $T^{0}, \ldots, T^{q}$ and a space $X$, we obtain a functor from $\mathcal{I}^{q+1}$ to spaces, which sends $\left(\mathbf{n}_{0}, \ldots, \mathbf{n}_{q}\right)$ to

$$
\Omega^{n_{0}+\cdots+n_{q}}\left(\left|T_{n_{q}}^{q} \wedge \cdots \wedge T_{n_{0}}^{0}\right| \wedge X\right) .
$$

Let $\mathcal{A}$ be a spectral category. Let $V(\mathcal{A}, X)_{n_{0}, \ldots, n_{q}}$ be the functor from $\mathcal{I}^{q+1}$ to spaces defined by

$$
\Omega^{n_{0}+\cdots+n_{q}}\left(\bigvee\left|\mathcal{A}\left(c_{q-1}, c_{q}\right)_{n_{q}} \wedge \cdots \wedge \mathcal{A}\left(c_{0}, c_{1}\right)_{n_{1}} \wedge \mathcal{A}\left(c_{q}, c_{0}\right)_{n_{0}}\right| \wedge X\right),
$$

where the sum $\bigvee$ is taken over the $(q+1)$-tuples $\left(q_{0}, \cdots, q_{n}\right)$ of objects of $\mathcal{A}$. Now define

$$
\operatorname{THH}_{q}(\mathcal{A})(X):=\operatorname{hocolim}_{\mathcal{I}^{q+1}} V(\mathcal{A}, X)_{n_{0}, \cdots, n_{q}} .
$$

This construction assembles into a simplicial space and we write $\operatorname{TH} H(\mathcal{A})(X)$ for its geometric realization. We obtain in this way a functor $\operatorname{THH}(\mathcal{A})(X)$ in the variable $X$. If we restrict ourselves to the spheres $S^{n}$, we obtain finally the symmetric spectrum of topological Hochschild homology of $\mathcal{A}$ denoted by $\operatorname{THH}(\mathcal{A})$. For the definition of topological cyclic homology involving the "cyclotomic" structure on $\operatorname{THH}(\mathcal{A})$, the author is invited to consult [2, Section 3]. We denote by $T C(\mathcal{A})$ the spectrum of topological cyclic homology of $\mathcal{A}$.

In order to compare dg categories with spectral ones, Blumberg and Mandell constructed in [2, Theorem A.2] a model structure on $\mathrm{Sp}^{\Sigma}$-Cat, whose weak equivalences are the spectral functors $F: \mathcal{A} \rightarrow \mathcal{B}$ which induce a bijection on the object sets and a stable equivalence

$$
F(x, y): \mathcal{A}(x, y) \longrightarrow \mathcal{B}(F x, F y), \quad x, y \in \mathcal{A}
$$


in $\mathrm{Sp}^{\Sigma}$. Using Shipley's zig-zag machinery [32], they constructed analogous variants of this model structure for categories enriched in dg modules, abelian spectra and $H \mathbb{Z}$-modules, and obtained in this way a functor from dg categories to spectral ones, see [2, Corollary A.3]. Given a dg category $\mathcal{A}$, its $T H H$ and $T C$ is by definition the pre-composition of the preceding functor with $T H H$ and $T C$ (for spectral categories). In conclusion, Blumberg and Mandell obtained well-defined functors

$$
T H H, T C: \text { dgcat } \longrightarrow \mathrm{Sp}^{\Sigma} \text {. }
$$

Remark 8.2 Notice that the comparison between dg categories and spectral ones established by Blumberg and Mandell makes use of Quillen model structures which don't have the correct weak equivalences (since these weak equivalences induce a bijection on the object sets). Therefore, it is not clear a priori if $T H H$ and $T C$ are invariant under quasi-equivalences. The zig-zag of Quillen equivalences of the previous Sections allow us to solve this problem.

\section{Proposition 8.3 The functors}

$$
T H H, T C: \text { dgcat } \longrightarrow \mathrm{Sp}^{\Sigma}
$$

send quasi-equivalences (see Example 2.10) to stable equivalences.

Proof By Blumberg and Mandell [2, Theorem 4.9] [2, Proposition 3.8], the functors

$$
\text { THH, TC: } \mathrm{Sp}^{\Sigma}-\mathrm{Cat} \longrightarrow \mathrm{Sp}^{\Sigma},
$$

send Dwyer-Kan equivalences (see Example 2.10) to weak equivalences and so descend to the homotopy category Ho(dgcat). Let $i: \mathbb{S} \rightarrow H \mathbb{Z}$ be the unique morphism of commutative symmetric ring spectra. By Proposition 5.21, we have the following restriction/extension of scalars Quillen adjunction

$$
\begin{gathered}
H \mathbb{Z}-\mathrm{Cat} \\
\left.i !\right|_{i} \\
\mathrm{Sp}^{\Sigma} \text {-Cat },
\end{gathered}
$$

with the right adjoint functor preserving (and reflecting) weak equivalences. Using the equivalence of the previous Section

$$
\mathbb{H}: \mathrm{Ho}(\text { dgcat }) \stackrel{\sim}{\longrightarrow} \mathrm{Ho}(H \mathbb{Z}-\mathrm{Cat}),
$$

which extends the one of Blumberg-Mandell, we conclude that the functors $T H H$ and $T C$ are invariant under quasi-equivalences. 
Remark 8.4 Using Thomason's hypercohomology construction [39], Geisser and Hesselholt introduced in a pioneer work [14] the topological Hochschild homology and topological cyclic homology of schemes and performed several important calculations, see for instance [14, Section 5.3].

More recently, Blumberg and Mandell defined in [2, Section 7] the topological Hochschild homology and the topological cyclic homology of a scheme $X$ by applying the functors

$$
T H H, T C: \text { dgcat } \longrightarrow \mathrm{Sp}^{\Sigma}
$$

to a dg category $\mathcal{D}_{\text {perf }}^{\mathrm{DG}}(X)$ modeling the derived category of perfect complexes of $\mathcal{O}_{X}$-modules. They proved that when $X$ is quasi-compact and semi-separated their definition coincides with the one of Geisser-Hesselholt.

Thanks to Proposition 8.3, we now have the "flexibility" of choosing for $\mathcal{D}_{\text {perf }}^{\mathrm{DG}}(X)$ any $\mathrm{dg}$ category which models the derived category of perfect complexes of $\mathcal{O}_{X}$-modules. For example, we can take for $\mathcal{D}_{\text {perf }}^{\mathrm{DG}}(X)$ the dg category of bounded complexes of injective $\mathcal{O}_{X}$-modules with coherent homology.

\subsection{Trace maps}

In this Subsection, we will use freely the language of derivators. The reader unfamiliar with this language is invited to consult Cisinski and Neeman [5, Section 1] or Grothendieck's original monograph [16].

Notice that the constructions of the previous Sections and of the previous Subsection furnishes us morphisms of derivators

$$
T H H, T C: \mathrm{HO}(\text { dgcat })_{Q E} \longrightarrow \mathrm{HO}\left(\mathrm{Sp}^{\Sigma}\right),
$$

from the derivator $\mathrm{HO}$ (dgcat) $Q E$ associated with the Quillen model structure described by Tabuada in [35, Theorem 1.8] to the derivator associated to the category of spectra. We now show that $T H H$ and $T C$ descend to the derivator $\mathrm{HO}$ (dgcat) associated with the Morita model structure described by Tabuada in [35, Theorem 2.27]. For this, we start by recalling some properties of $T H H$ and $T C$ in the context of spectral categories.

Definition 8.5 (see Blumberg and Mandell [2, Section 4]) A spectral functor $F: \mathcal{C} \rightarrow$ $\mathcal{D}$ is a Morita equivalence if:

- for all objects $x, y \in \mathcal{C}$, the morphism

$$
F(x, y): \mathcal{C}(x, y) \longrightarrow \mathcal{D}(F x, F y)
$$

is a stable equivalence in $\mathrm{Sp}^{\Sigma}$ and 
- the smallest thick (that is, closed under direct factors) triangulated subcategory of $\mathrm{Ho}(\mathcal{D}-\mathrm{Mod})$ (see Remark 2.12) generated by the image of $F$ is equivalent to the full subcategory of compact objects in $\mathrm{Ho}(\mathcal{D}-\mathrm{Mod})$.

Remark 8.6 Thanks to Blumberg and Mandell [2, Definition 4.12] [2, Proposition 3.8], if a spectral functor $F$ is a Morita equivalence, then $\operatorname{THH}(F)$ and $T C(F)$ are weak equivalences.

Let us now return to the context of dg categories.

Proposition 8.7 Let $F: \mathcal{A} \rightarrow \mathcal{B}$ be a Morita dg functor, see Tabuada [35, Section 2.5]. Then the associated spectral functor $i^{*} \mathbb{H}(F)$ is a Morita equivalence.

Proof Thanks to Tabuada [35, Propositions 2.14 and 2.35], $F$ is a Morita dg functor if and only if $F_{f}$ is a quasi-equivalence, where $(-)_{f}$ denotes a functorial fibrant resolution functor

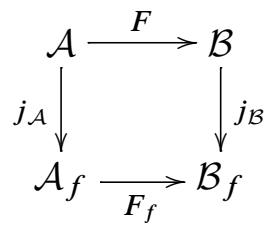

in the Quillen model structure described by Tabuada in [35, Theorem 2.27]. By applying the composed functor $i^{*} \mathbb{H}$ to the above diagram, we obtain a diagram of spectral categories

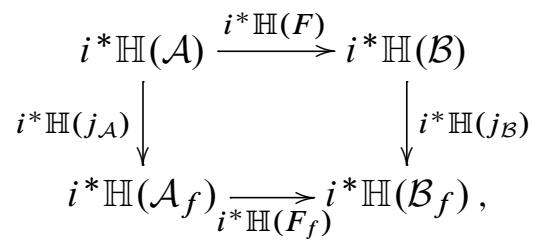

with $i^{*} \mathbb{H}\left(F_{f}\right)$ a quasi-equivalence (and so a Morita equivalence). Moreover, since $j_{\mathcal{A}}$ and $j_{\mathcal{B}}$ are fully faithful Morita dg functors with values in Morita fibrant dg categories (see Tabuada [35, Proposition 2.34]), [2, Proposition 4.8] implies that $i^{*} \mathbb{H}\left(j_{\mathcal{A}}\right)$ and $i^{*} \mathbb{H}\left(j_{\mathcal{B}}\right)$ are Morita equivalences. In conclusion, by the two out of three property, the spectral functor $i^{*} \mathbb{H}(F)$ is also a Morita equivalence.

Remark 8.8 By Remark 8.6 and Proposition 8.7 we obtain well-defined morphisms of derivators

$$
T H H, T C: \mathrm{HO}(\text { dgcat }) \longrightarrow \mathrm{HO}\left(\mathrm{Sp}^{\Sigma}\right) \text {. }
$$


Proposition 8.9 The morphisms of derivators $T H H$ and $T C$ are additive invariants of dg categories, see Tabuada [35, Terminology 3.86].

Proof The proof will consist on verifying that $T H H$ and $T C$ satisfy all the conditions of Tabuada [35, Terminology 3.86]. Clearly $T H H$ and $T C$ preserve the point. Moreover, by construction the morphisms of derivators

$$
i^{*}: \mathrm{HO}(H \mathbb{Z}-\mathrm{Cat}) \longrightarrow \mathrm{HO}\left(\mathrm{Sp}^{\Sigma}-\mathrm{Cat}\right)
$$

and

$$
T H H, T C: \mathrm{HO}\left(\mathrm{Sp}^{\Sigma}-\mathrm{Cat}\right) \longrightarrow \mathrm{HO}\left(\mathrm{Sp}^{\Sigma}\right)
$$

commute with filtered homotopy colimits. It remains to show that $T H H$ and $T C$ satisfy the additivity condition A). Let

$$
0 \longrightarrow \mathcal{A} \underset{i_{\mathcal{A}}}{\stackrel{R}{\leftrightarrows}} \mathcal{B} \stackrel{i_{\mathcal{C}}}{\longleftrightarrow} \mathcal{C} \longrightarrow 0
$$

be a split short exact sequence of dg categories, that is, $P \circ i_{\mathcal{A}}=0, R$ is a dg functor right adjoint to $i_{\mathcal{A}}, i_{\mathcal{C}}$ is a dg functor right adjoint to $P$ and we have $P \circ i_{\mathcal{C}}=\operatorname{Id}_{\mathcal{C}}$ and $R \circ i_{\mathcal{A}}=\operatorname{Id}_{\mathcal{A}}$ via the adjunction morphisms, see Tabuada [35, Definition 3.68]. Observe that by applying the functor $i^{*} \mathbb{H}(-)$ to the previous short exact sequence of dg categories, we obtain a split short exact sequence of spectral categories (see Blumberg and Mandell [2, Section 6])

$$
0 \longrightarrow i^{*} \mathbb{H}(\mathcal{A}) \longleftrightarrow i^{*} \mathbb{H}(\mathcal{B}) \longleftrightarrow i^{*} \mathbb{H}(\mathcal{C}) \longrightarrow 0 \text {. }
$$

By the localization Theorem (see Blumberg and Mandell [2, Theorem 6.1]), we obtain split triangles

$$
\operatorname{THH}(\mathcal{A}) \longleftrightarrow T H H(\mathcal{B}) \longleftrightarrow T H H(\mathcal{C}) \longrightarrow T H H(\mathcal{A})[1]
$$

and

$$
T C(\mathcal{A}) \longleftrightarrow T C(\mathcal{B}) \longleftrightarrow T C(\mathcal{C}) \longrightarrow T C(\mathcal{A})[1]
$$

in $\mathrm{Ho}\left(\mathrm{Sp}^{\Sigma}\right)$. In conclusion, the dg functors $i_{\mathcal{A}}$ and $i_{\mathcal{C}}$ induce isomorphisms

$$
\begin{gathered}
T H H(\mathcal{A}) \oplus T H H(\mathcal{C}) \stackrel{\sim}{\longrightarrow} T H H(\mathcal{B}), \\
T C(\mathcal{A}) \oplus T C(\mathcal{C}) \stackrel{\sim}{\longrightarrow} T C(\mathcal{B})
\end{gathered}
$$

in $\mathrm{Ho}\left(\mathrm{Sp}^{\Sigma}\right)$ and so the proof is finished. 
We now construct trace maps from algebraic $K$-theory to topological Hochschild homology. Let $K(-)$ denote the Waldhausen's $K$-theory spectrum functor of dg categories, see Tabuada [35, Definition 2.6.2] and Schwede [28, Example 2.11-I]. Since $K(-)$ satisfies additivity (see Waldhausen [40, Section 1.4]) it gives rise to an additive invariant of dg categories (see Tabuada [35, Terminology 3.86])

$$
K(-): \mathrm{HO}(\text { dgcat }) \longrightarrow \mathrm{HO}\left(\mathrm{Sp}^{\Sigma}\right) \text {. }
$$

Let

$$
E(-): \mathrm{HO}(\text { dgcat }) \longrightarrow \mathrm{HO}\left(\mathrm{Sp}^{\Sigma}\right)
$$

be an additive invariant of $\mathrm{dg}$ categories. By Tabuada [35, Theorem 3.85], E(-) descends to the additive motivator of dg categories $\mathcal{M}_{\mathrm{dg}}^{\mathrm{add}}$, inducing a morphism

$$
\bar{E}(-): \mathcal{M}_{\mathrm{dg}}^{\mathrm{add}} \longrightarrow \mathrm{HO}\left(\mathrm{Sp}^{\Sigma}\right)
$$

between two triangulated derivators. Since $K(-)$ is also an additive invariant it induces also a morphism

$$
\bar{K}(-): \mathcal{M}_{\mathrm{dg}}^{\mathrm{add}} \longrightarrow \mathrm{HO}\left(\mathrm{Sp}^{\Sigma}\right) .
$$

Thanks to Tabuada [36, Theorem 15.10], the morphism $\bar{K}(-)$ is co-represented by the object $\mathcal{U}_{a}(\mathbb{Z})$ in $\mathcal{M}_{\mathrm{dg}}^{\text {add }}$. This implies, by the enriched Yoneda Lemma, that we have a natural isomorphism in the stable homotopy category of spectra

$$
\mathrm{Nat}^{\mathrm{Sp}^{\Sigma}}(\bar{K}(-), \bar{E}(-)) \simeq \bar{E}(\mathbb{Z})=E(\mathbb{Z}),
$$

where $\mathrm{Nat}^{\mathrm{Sp}^{\Sigma}}(-,-)$ denotes the symmetric spectrum of natural transformations. Since by Tabuada [35, Theorem 3.82] we have the following identifications

$$
\pi_{0}^{s} \mathrm{Nat}^{\mathrm{Sp}^{\Sigma}}(\bar{K}(-), \bar{E}(-))=\operatorname{Nat}(\bar{K}(-), \bar{E}(-)) \simeq \operatorname{Nat}(K(-), E(-)),
$$

we obtain a natural isomorphism

$$
\operatorname{Nat}(K(-), E(-)) \simeq \pi_{0}^{s} E(\mathbb{Z})
$$

Proposition 8.10 We have non-trivial natural transformations of morphisms of derivators

$$
\begin{aligned}
& \text { (i): } K(-) \Rightarrow T H H(-) \\
& \text { (ii): } K(-) \Rightarrow T H H[-(2 r-1)](-), \quad r \geq 1
\end{aligned}
$$

where THH $[-(2 r-1)](-)$ denotes the composition of THH $(-)$ with the $(-(2 r-1))-$ suspension functor in $\mathrm{HO}\left(\mathrm{Sp}^{\Sigma}\right)$. 
Corollary 8.11 By applying the functors

$$
\pi_{n}^{s}: \operatorname{Ho}\left(\mathrm{Sp}^{\Sigma}\right) \longrightarrow \operatorname{Mod}-\mathbb{Z}, n \geq 0
$$

to the natural transformations of Proposition 8.10, we obtain non-trivial natural transformations of functors

$$
\begin{aligned}
& \text { (i): } K_{n}(-) \Rightarrow T H H_{n}(-), \quad n \geq 0 \\
& \text { (ii): } K_{n}(-) \Rightarrow T H H_{n+2 r-1}(-), \quad n \geq 0, r \geq 1 .
\end{aligned}
$$

Proof (of Proposition 8.10) Thanks to Bökstedt, we have the following calculation (see Dundas, Goodwillie and McCarthy [10, Section 0.2.3])

$$
\operatorname{THH}_{j}(\mathbb{Z})= \begin{cases}\mathbb{Z} & \text { if } j=0 \\ \mathbb{Z} / r \mathbb{Z} & \text { if } j=2 r-1, r \geq 1 \\ 0 & \text { otherwise } .\end{cases}
$$

Therefore, if in the previous isomorphism (see (1)) we take $E(-)=T H H(-)$, the canonical generator of $\mathbb{Z}$ furnishes us automatically the natural transformation of item (i). If in the previous isomorphism (see (1)) we take $E(-)=T H H[-(2 r-1)](-), r \geq 1$, the canonical generator of $\mathbb{Z} / r \mathbb{Z}$ furnishes us automatically the natural transformation of item (ii). The non-triviality of the natural transformations follows from its own construction.

Proposition 8.12 Let $p$ be a prime number. If we work over the ring $\mathbb{Z} / p \mathbb{Z}$ we have non-trivial natural transformations of morphisms of derivators

$$
K(-) \Rightarrow T H H[-2 r](-), \quad r \geq 0
$$

and so non-trivial natural transformations of functors

$$
K_{n}(-) \Rightarrow T H H_{n+2 r}(-), \quad n, r \geq 0 .
$$

Proof The proof is the same as the one of Proposition 8.10 , but over $\mathbb{Z} / p \mathbb{Z}$ we have the following calculation

$$
T H H_{j}(\mathbb{Z} / p \mathbb{Z})= \begin{cases}\mathbb{Z} / p \mathbb{Z} & \text { if } j \text { is even } \\ 0 & \text { if } j \text { is odd. }\end{cases}
$$

(See Dundas, Goodwillie and McCarthy [10, Section 0.2.3].)

Remark 8.13 Given a scheme $X$, Geisser and Hesselholt constructed in [14] the cyclotomic trace map from the algebraic $K$-theory of $X$ to its topological Hochschild homology and topological cyclic homology. This cyclotomic trace map was one of the main working tools which allowed them to make extensive $K$-theoretic computations, 
see for instance [14, Section 5]. Recall from Remark 8.4 that when $X$ is quasicompact and semi-separated, Blumberg-Mandell's definition coincides with the one of Geisser-Hesselholt. This implies that there are well-defined cyclotomic trace maps from the algebraic $K$-theory of $X$ to $\operatorname{THH}\left(\mathcal{D}_{\text {perf }}^{\mathrm{DG}}(X)\right)$ and $T C\left(\mathcal{D}_{\text {perf }}^{\mathrm{DG}}(X)\right)$. Blumberg and Mandell improved this by giving in [2, Appendix B] an intrinsic construction of the cyclotomic trace for certain dg categories like $\mathcal{D}_{\text {perf }}^{\mathrm{DG}}(X)$, which come from complicial Waldhausen ones.

In our case, the natural transformations of Proposition 8.10 have the advantage of being available for all $\mathrm{dg}$ categories but the disadvantage of being defined only over $\mathbb{Z}$ (or over $\mathbb{Z} / p \mathbb{Z}$ with $p$ prime). At the present time the author does not know how to relate these natural transformations with the cyclotomic trace. The reason for this is that, in contrast with Geisser-Hesselholt and Blumberg-Mandell's work, our construction is given in an implicit way (making use of a co-representability result, see Tabuada [36, Theorem 15.10]). However, taking into account the naturality of all these constructions, it is expected that in the particular case of a quasi-compact and separated scheme $X$ they all coincide. This will be the subject of future research.

\section{Topological equivalence theory}

In this Section we recall Dugger-Shipley's notion of topological equivalence [9] and show that over the field of rational numbers $\mathbb{Q}$, two dg categories are topologically equivalent if and only if they are quasi-equivalent (see Proposition 9.5).

Let $i: \mathbb{S} \rightarrow H \mathbb{Z}$ be the unique morphism of commutative symmetric ring spectra. By Proposition 5.21 we have the restriction/extension of scalars Quillen adjunction

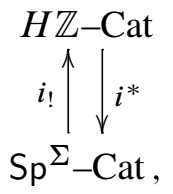

with respect to the mode structure of Theorem 5.17.

Definition 9.1 Let $\mathcal{A}$ and $\mathcal{B}$ be two dg categories. We say that $\mathcal{A}$ and $\mathcal{B}$ are topologically equivalent if the spectral categories $i^{*}\left(\mathbb{H}(\mathcal{A})\right.$ ) and $i^{*}(\mathbb{H}(\mathcal{B})$ ) (see Notation 7.1) are isomorphic in $\mathrm{Ho}\left(\mathrm{Sp}^{\Sigma}-\mathrm{Cat}\right)$.

Remark 9.2 If $\mathcal{A}$ and $\mathcal{B}$ have a single object, Definition 9.1 corresponds to DuggerShipley's notion of topological equivalence for differential graded algebras, see [9]. Moreover, the zig-zag of Quillen equivalences described in Section 7 shows that if $\mathcal{A}$ and $\mathcal{B}$ are isomorphic in $\mathrm{Ho}$ (dgcat) then they are topologically equivalent. 
From now on we will be working over $\mathbb{Q}$. We will denote by dgcat $t_{\mathbb{Q}}$ the category of small dg categories defined over $\mathbb{Q}$. We have the restriction/extension of scalars Quillen adjunction

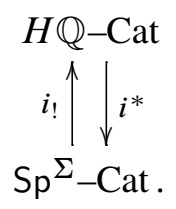

Definition 9.3 Let $\mathcal{A}$ be a spectral category. We say that $\mathcal{A}$ satisfies condition (RH) if for all objects $x, y \in \mathcal{A}$, the symmetric spectrum $\mathcal{A}(x, y)$ has rational homotopy.

Lemma 9.4 Let $F: \mathcal{A} \rightarrow \mathcal{B}$ be a stable quasi-equivalence between spectral categories which satisfying condition $(R H)$. Then the $H \mathbb{Q}$-functor $i_{!}(F)$ is a stable quasiequivalence (see Definition 5.8).

Proof Dugger-Shipley's proof of [9, Proposition 1.7] implies that for all objects $x, y \in \mathcal{A}$, the morphism

$$
i_{!}(\mathcal{A})(x, y)=\mathcal{A}(x, y) \wedge_{\mathbb{S}} H \mathbb{Q} \longrightarrow \mathcal{B}(F x, F y) \wedge \mathbb{S} H \mathbb{Q}=i_{!}(\mathcal{B})(F x, F y)
$$

is a projective stable equivalence in $H \mathbb{Q}$-Mod. Consider the commutative square

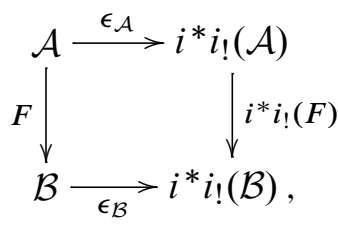

where $\epsilon_{\mathcal{A}}$ and $\epsilon_{\mathcal{B}}$ are the co-units of the adjunction $\left(i_{!}, i^{*}\right)$. Since $\mathcal{A}$ and $\mathcal{B}$ satisfy condition (RH), the proof of [9, Proposition 1.7] implies also that for all objects $x, y \in \mathcal{A}$, the morphism

$$
\mathcal{A}(x, y) \longrightarrow \mathcal{A}(x, y) \wedge_{\mathbb{S}} H \mathbb{Q}=i^{*} i_{!}(\mathcal{A})(x, y)
$$

is a projective stable equivalence in $H \mathbb{Q}-\operatorname{Mod}$. Since $\epsilon_{\mathcal{A}}$ and $\epsilon_{\mathcal{B}}$ induce the identity map on the object sets, we conclude that $\epsilon_{\mathcal{A}}$ and $\epsilon_{\mathcal{B}}$ are stable quasi-equivalences. By Proposition 5.11 we conclude that $i_{!}(P)$ is a stable quasi-equivalence.

Proposition 9.5 Let $\mathcal{A}$ and $\mathcal{B}$ be two dg categories over $\mathbb{Q}$. Then $\mathcal{A}$ and $\mathcal{B}$ are

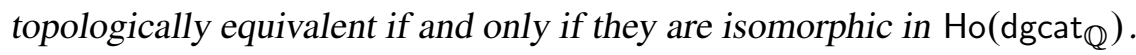


Proof By Remark 9.2, it is enough to prove that if $\mathcal{A}$ and $\mathcal{B}$ are topologically equivalent then they are isomorphic in $\mathrm{Ho}\left(\operatorname{dgcat}_{\mathbb{Q}}\right)$. If $\mathcal{A}$ and $\mathcal{B}$ are topologically equivalent then there exists a zig-zag of stable quasi-equivalences in $\mathrm{Sp}^{\Sigma}$-Cat

$$
i^{*}(\mathcal{A}) \stackrel{\sim}{\leftarrow} \mathcal{Z}_{1} \stackrel{\sim}{\rightarrow} \cdots \stackrel{\sim}{\rightarrow} \mathcal{Z}_{n} \stackrel{\sim}{\leftarrow} i^{*}(\mathcal{B})
$$

relating $i^{*}(\mathcal{A})$ and $i^{*}(\mathcal{B})$. By applying the functor $i_{\text {! }}$ to this zig-zag, we obtain by Lemma 9.4 the following zig-zag

$$
i_{!} i^{*}(\mathcal{A}) \stackrel{\sim}{\leftarrow} i_{!}\left(\mathcal{Z}_{1}\right) \stackrel{\sim}{\rightarrow} \cdots \stackrel{\sim}{\rightarrow} i_{!}\left(\mathcal{Z}_{n}\right) \stackrel{\sim}{\leftarrow} i_{!} i^{*}(\mathcal{B})
$$

of stable quasi-equivalences in $H \mathbb{Q}-$ Cat. We now show that the units of the adjunction $\left(i_{!}, i^{*}\right)$

$$
\eta_{\mathcal{A}}: i_{!} i^{*}(\mathcal{A}) \rightarrow \mathcal{A} \quad \eta_{\mathcal{B}}: i_{!} i^{*}(\mathcal{B}) \rightarrow \mathcal{B}
$$

are stable quasi-equivalences. Notice that Dugger-Shipley's proof of [9, Proposition 1.7] implies that for all objects $x, y \in \mathcal{A}$, the morphism

$$
i_{!} i^{*}(\mathcal{A})(x, y) \longrightarrow \mathcal{A}(x, y)
$$

is a projective stable equivalence in $H \mathbb{Q}$-Mod. Since $\eta_{\mathcal{A}}$ induces the identity map on the object sets, we conclude that it satisfies condition (WE2) and so that it is a stable quasi-equivalence. An analogous result holds for $\mathcal{B}$, and so the proof is finished.

\section{Appendix A Adjunctions}

Let $\left(\mathcal{C},-\otimes-, \mathbf{1}_{\mathcal{C}}\right)$ and $\left(\mathcal{D},-\wedge-, \mathbf{1}_{\mathcal{D}}\right)$ be two symmetric monoidal categories and

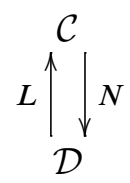

an adjunction, with $N$ a lax monoidal functor (see Definition 2.4).

Notation A.1 If $I$ is a set, we denote by $\mathcal{C}^{I}-\mathrm{Gr}$, resp. by $\mathcal{C}^{I}-$ Cat, the category of $\mathcal{C}$-graphs with a fixed set of objects $I$, resp. the category of categories enriched over $\mathcal{C}$ which have a fixed set of objects $I$. The morphisms in $\mathcal{C}^{I}-\mathrm{Gr}$ and $\mathcal{C}^{I}-$ Cat induce the identity map on the objects. 
We have a natural adjunction

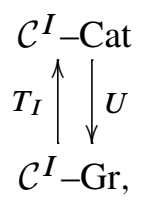

where $U$ is the forgetful functor and $T_{I}$ is defined as

$$
T_{I}(\mathcal{A})(x, y):=\left\{\begin{array}{cc}
\mathbf{1}_{\mathcal{C}} \amalg \underset{x, x_{1}, \ldots, x_{n}, y}{\amalg} \mathcal{A}\left(x, x_{1}\right) \otimes \cdots \otimes \mathcal{A}\left(x_{n}, y\right) & \text { if } x=y \\
\bigsqcup_{x, x_{1}, \ldots, x_{n}, y} \mathcal{A}\left(x, x_{1}\right) \otimes \cdots \otimes \mathcal{A}\left(x_{n}, y\right) & \text { if } x \neq y
\end{array}\right.
$$

Composition is given by concatenation and the unit corresponds to $\mathbf{1}_{\mathcal{C}}$.

Remark A.2 If the category $\mathcal{C}$ carries a cofibrantly generated Quillen model structure, the categories $\mathcal{C}^{I}-\mathrm{Gr}$ and $\mathcal{C}^{I}$-Cat admit standard model structures, see Schwede and Shipley [30, Section 6]. The weak equivalences (resp. fibrations) are the morphisms $F: \mathcal{A} \rightarrow \mathcal{B}$ such that

$$
F(x, y): \mathcal{A}(x, y) \longrightarrow \mathcal{B}(x, y), \quad x, y \in \mathcal{A}
$$

is a weak equivalence (resp. fibration) in $\mathcal{C}$. In fact, the Quillen model structure on $\mathcal{C}$ naturally induces a model structure on $\mathcal{C}^{I}-\mathrm{Gr}$, which can be lifted along the functor $T_{I}$ using the lifting Theorem (see Hirschhorn [20, Theorem 11.3.2]).

The adjunction $(L, N)$ induces the following one

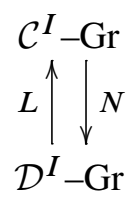

which we still denoted by $(L, N)$. Since the functor $N: \mathcal{C} \rightarrow \mathcal{D}$ is lax monoidal it induces, as shown by Schwede and Shipley in [30, Section 3.3], a functor

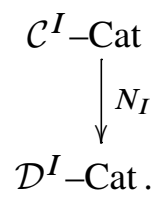

More precisely, let $\mathcal{B} \in \mathcal{C}^{I}-$ Cat and $x, y$ and $z$ objects of $\mathcal{B}$. Then $N_{I}(\mathcal{B})$ has the same objects as $\mathcal{B}$, and its spaces of morphisms are given by

$$
N_{I}(\mathcal{B})(x, y):=N \mathcal{B}(x, y), \quad x, y \in \mathcal{B}
$$


with the composition defined by

$$
N \mathcal{B}(x, y) \wedge N \mathcal{B}(y, z) \longrightarrow N(\mathcal{B}(x, y) \otimes \mathcal{B}(y, z)) \stackrel{N(c)}{\longrightarrow} N \mathcal{B}(x, z),
$$

where $c$ denotes the composition operation in $\mathcal{B}$. As shown by Schwede and Shipley [30, Section 3.3] the functor $N_{I}$ admits a left adjoint $L_{I}$ : let $\mathcal{A} \in \mathcal{D}^{I}-$ Cat. The value of the left adjoint $L_{I}$ on $\mathcal{A}$ is defined as the coequalizer of two morphisms in $\mathcal{C}^{I}$-Cat

$$
T_{I} L U T_{I} U(\mathcal{A}) \underset{\psi_{2}}{\stackrel{\psi_{1}}{\longrightarrow}} T_{I} L U(\mathcal{A}) \longrightarrow L_{I}(\mathcal{A}) .
$$

The morphism $\psi_{1}$ is obtained from the unit of the adjunction

$$
T_{I} U \mathcal{A} \longrightarrow \mathcal{A},
$$

by applying the composite functor $T_{I} L U$; the morphism $\psi_{2}$ is the unique morphism in $\mathcal{C}^{I}$-Cat induced by the $\mathcal{C}^{I}-$ Gr morphism

$$
L U T_{I} U(\mathcal{A}) \longrightarrow U T_{I} L U(\mathcal{A})
$$

whose value at $L U T_{I} U(\mathcal{A})(x, y), x, y \in I$ is given by

$$
\begin{gathered}
\bigsqcup_{x, x_{1}, \ldots, x_{n}, y} L\left(\mathcal{A}\left(x, x_{1}\right) \otimes \cdots \otimes \mathcal{A}\left(x_{n}, y\right)\right) \\
\downarrow_{\phi} \\
\underset{x, x_{1}, \ldots, x_{n}, y}{\bigsqcup} L \mathcal{A}\left(x, x_{1}\right) \otimes \cdots \otimes L \mathcal{A}\left(x_{n}, y\right),
\end{gathered}
$$

where $\phi$ is the lax comonoidal structure on $L$ induced by the lax monoidal structure on $N$, see Schwede and Shipley [30, Section 3.3].

Remark A.3 Notice that if $\mathcal{C}$ and $\mathcal{D}$ are Quillen model categories and the adjunction $(L, N)$ is a weak monoidal Quillen equivalence (see Definition 2.5), SchwedeShipley's [30, Proposition 6.4] furnishes us a Quillen equivalence

$$
\begin{gathered}
\mathcal{C}^{I}-\text { Cat } \\
L_{I} \uparrow{ }^{N_{I}} \\
\mathcal{D}^{I}-\text { Cat }
\end{gathered}
$$

with respect to the model structure of Remark A.2. Moreover, if $L$ is strong monoidal (see Definition 2.4), the left adjoint $L_{I}$ is given by the original functor $L$. 
Left adjoint Notice that the functor $N_{I}: \mathcal{C}^{I}-\mathrm{Cat} \rightarrow \mathcal{D}^{I}-$ Cat, of the previous Section, can be naturally defined for every set $I$ and so it induces a "global" functor

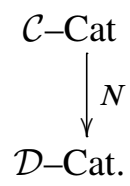

In this Section we will construct its left adjoint: let $\mathcal{A} \in \mathcal{D}$-Cat and denote by $I$ its set of objects. Define $L_{\text {cat }}(\mathcal{A})$ as the $\mathcal{C}$-category $L_{I}(\mathcal{A})$. Now, let $F: \mathcal{A} \rightarrow \mathcal{A}^{\prime}$ be a $\mathcal{D}$-functor. We denote by $I^{\prime}$ the set of objects of $\mathcal{A}^{\prime}$. The $\mathcal{D}$-functor $F$ induces the following diagram in $\mathcal{C}$-Cat:

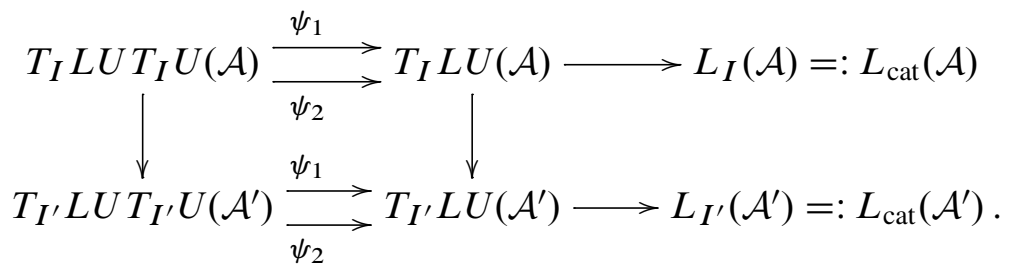

Notice that the left square with horizontal arrows $\psi_{1}$ (resp. $\psi_{2}$ ) is commutative. Since the inclusions

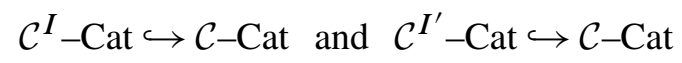

preserve coequalizers the previous diagram in $\mathcal{C}$-Cat induces a $\mathcal{C}$-functor

$$
L_{\text {cat }}(F): L_{\text {cat }}(\mathcal{A}) \longrightarrow L_{\text {cat }}\left(\mathcal{A}^{\prime}\right) .
$$

In conclusion, we have constructed a functor

$$
L_{\text {cat }}: \mathcal{D}-\text { Cat } \longrightarrow \mathcal{C}-\text { Cat. }
$$

Proposition A.4 (see Tabuada [38, Proposition 5.5]) The functor $L_{\text {cat }}$ is left adjoint to $N$.

Remark A.5 (see Schwede and Shipley [30, Section 3.3]) Notice that if, in the initial adjunction $(L, N)$, the left adjoint $L$ is strong monoidal (see Definition 2.4), Remark A.3 implies that the left adjoint functor

$$
L_{\text {cat }}: \mathcal{D}-\text { Cat } \longrightarrow \mathcal{C}-\text { Cat }
$$

is given by the original left adjoint $L$. 


\section{Appendix B Bousfield localization techniques}

In this Appendix we recall and generalize some results concerning the construction and localization of Quillen model structures. We start by stating a weaker form of the Bousfield localization Theorem (see Goerss and Jardine [15, Theorem 4.1-X]).

Definition B.1 Let $\mathcal{M}$ be a Quillen model category, $Q: \mathcal{M} \rightarrow \mathcal{M}$ a functor and $\eta: \mathrm{Id} \rightarrow Q$ a natural transformation between the identity functor and $Q$. A morphism $f: A \rightarrow B$ in $\mathcal{M}$ is:

- a $Q$-weak equivalence if $Q(f)$ is a weak equivalence in $\mathcal{M}$.

- a cofibration if it is a cofibration in $\mathcal{M}$.

- a $Q$-fibration if it has the RLP with respect to all cofibrations which are $Q$-weak equivalences.

An immediate analysis of the proof of [15, Theorem 4.1-X] allow us to state the following general Theorem B.2. Notice that in its entire proof, it is only used the right properness of $\mathcal{M}$ and in the proof of [15, Lemma 4.6-X] and of [15, Theorem 4.8-X] it is only used condition (A3).

Theorem B.2 (see Goerss and Jardine [15, Theorem 4.1-X]) Let $\mathcal{M}$ be a right proper Quillen model structure, $Q: \mathcal{M} \rightarrow \mathcal{M}$ a functor and $\eta: \mathrm{Id} \rightarrow Q$ a natural transformation such that the following three conditions hold:

(A1) The functor $Q$ preserves weak equivalences.

(A2) The maps $\eta_{Q(A)}, Q\left(\eta_{A}\right): Q(A) \rightarrow Q Q(A)$ are weak equivalences in $\mathcal{M}$.

(A3) Given a diagram

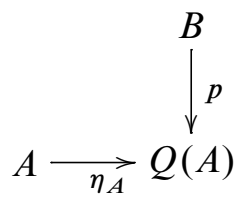

with $p$ a $Q$-fibration, the induced map $\eta_{\mathcal{A}_{*}}: A \times{ }_{Q(A)} B \rightarrow B$ is a $Q$-weak equivalence.

Then there is a right proper Quillen model structure on $\mathcal{M}$ for which the weak equivalences are the $Q$-weak equivalences, the cofibrations those of $\mathcal{M}$ and the fibrations the $Q$-fibrations. 
Theorem B.3 (see Goerss and Jardine [15, Theorem 4.8-X]) Suppose that the right proper Quillen model category $\mathcal{M}$ and the functor $Q$ together satisfy the conditions for Theorem B.2. Then a map $f: A \rightarrow B$ is a $Q$-fibration if and only if it is a fibration in $\mathcal{M}$ and the square

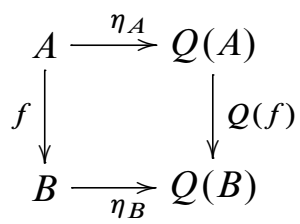

is homotopy cartesian in $\mathcal{M}$.

Corollary B.4 (see Goerss and Jardine [15, Corollary 4.12-X]) Suppose that the right proper Quillen model category $\mathcal{M}$ and the functor $Q$ together satisfy the conditions for Theorem B.2. Then an object $A$ of $\mathcal{M}$ is $Q$-fibrant if and only if it is fibrant in $\mathcal{M}$ and the map $\eta_{A}: A \rightarrow Q(A)$ is a weak equivalence in $\mathcal{M}$.

\section{Appendix C Non-additive filtration argument}

Let $\mathcal{D}$ be a closed symmetric monoidal category. We denote by $-\wedge-$ its symmetric monoidal product and by $\mathbf{1}$ its unit. In this Appendix we start by constructing a functor $U$, which associates to every object $X \in \mathcal{D}$ a gentle $\mathcal{D}$-category $U(X)$. By gentle we mean that the data of a $\mathcal{D}$-functor $U(X) \rightarrow \mathcal{A}$ corresponds to the choice of two objects $x$ and $y$ in $\mathcal{A}$ plus a morphism $X \rightarrow \mathcal{A}(x, y)$ in $\mathcal{D}$, see Remark C.2. When $\mathcal{D}$ is a cofibrantly generated model category, the functor $U$ will allow us to lift generating (trivial) cofibrations in $\mathcal{D}$ to generating (trivial) cofibrations in $\mathcal{D}$-Cat, see for instance Theorem 3.19 and 5.5. In Propositions C.3 and C.4 we will analyse certain pushouts in $\mathcal{D}$-Cat along $\mathcal{D}$-functors of shape $U(j): U(K) \rightarrow U(L)$, with $j: K \rightarrow L$ a (trivial) cofibration in $\mathcal{D}$.

Definition C.1 Consider the functor

$$
U: \mathcal{D} \longrightarrow \mathcal{D}-\text { Cat },
$$

which sends an object $X \in \mathcal{D}$ to the $\mathcal{D}$-category $U(X)$, with two objects 1 and 2 and such that $U(X)(1,1)=U(X)(2,2)=\mathbf{1}, U(X)(1,2)=X$ and $U(X)(2,1)=0$, where 0 denotes the initial object in $\mathcal{D}$. Composition is naturally defined (notice that 0 acts as a zero with respect to $\wedge$ since the bi-functor $-\wedge-$ preserves colimits in each of its variables). 
Remark C.2 For an object $X \in \mathcal{D}$, the object $U(X) \in \mathcal{D}$-Cat co-represents the following functor

$$
\begin{aligned}
& \mathcal{D} \text {-Cat } \longrightarrow \quad \text { Set } \\
& \mathcal{A} \mapsto \coprod_{(x, y) \in \mathcal{A} \times \mathcal{A}} \operatorname{Hom}_{\mathcal{D}}(X, \mathcal{A}(x, y)) .
\end{aligned}
$$

This shows us, in particular, that the functor $U$ preserves sequentially small objects.

Now, suppose that $\mathcal{D}$ is a monoidal model category with cofibrant unit which satisfies the monoid axiom, see Schwede and Shipley [29, Definition 3.3]. In what follows, by smash product we mean the symmetric product $-\wedge-$ of $\mathcal{D}$.

Proposition C.3 Let $\mathcal{A}$ be a $\mathcal{D}$-category, $j: K \rightarrow L$ a trivial cofibration in $\mathcal{D}$ and $U(K) \stackrel{F}{\rightarrow} \mathcal{A}$ a morphism in $\mathcal{D}-C a t$. Then, in the pushout

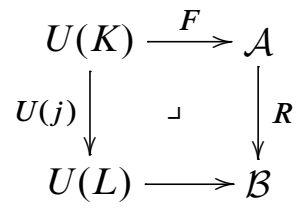

the morphisms

$$
R(x, y): \mathcal{A}(x, y) \longrightarrow \mathcal{B}(x, y), \quad x, y \in \mathcal{A}
$$

are weak equivalences in $\mathcal{D}$.

Proof Notice that $\mathcal{A}$ and $\mathcal{B}$ have the same set of objects and the morphism $R$ induces the identity on sets of objects. The description of the morphisms

$$
R(x, y): \mathcal{A}(x, y) \rightarrow \mathcal{B}(x, y), \quad x, y \in \mathcal{A}
$$

in $\mathcal{D}$ is analogous to the one given by Schwede and Shipley in [29, Lemma 6.2]. The idea is to think of $\mathcal{B}(x, y)$ as consisting of formal smash products of elements in $L$ with elements in $\mathcal{A}$, with the relations coming from $K$ and the composition in $\mathcal{A}$. Consider the same (conceptual) proof as the one of [29, Lemma 6.2]: $\mathcal{B}(x, y)$ will appear as the colimit in $\mathcal{D}$ of a sequence

$$
\mathcal{A}(x, y)=P_{0} \rightarrow P_{1} \rightarrow \cdots \rightarrow P_{n} \rightarrow \cdots,
$$

that we now describe. We start by defining a $n$-dimensional cube in $\mathcal{D}$, that is, a functor

$$
W: \mathcal{P}(\{1,2, \ldots, n\}) \longrightarrow \mathcal{D}
$$


from the poset category of subsets of $\{1,2, \ldots, n\}$ to $\mathcal{D}$. If $S \subseteq\{1,2, \ldots, n\}$ is a subset, the vertex of the cube at $S$ is

$$
W(S):=\mathcal{A}(x, F(0)) \wedge C_{1} \wedge \mathcal{A}(F(1), F(1)) \wedge C_{2} \wedge \cdots \wedge C_{n} \wedge \mathcal{A}(F(1), y),
$$

with

$$
C_{i}=\left\{\begin{array}{l}
K \text { if } i \notin S \\
L \text { if } i \in S
\end{array}\right.
$$

The maps in the cube $W$ are induced from the map $j: K \rightarrow L$ and the identity on the remaining factors. So at each vertex, a total of $n+1$ factors of objects in $\mathcal{D}$, alternate with $n$ smash factors of either $K$ or $L$. The initial vertex, corresponding to the empty subset has all its $C_{i}$ 's equal to $K$, and the terminal vertex corresponding to the whole set has all it's $C_{i}$ 's equal to $L$.

Denote by $Q_{n}$, the colimit of the punctured cube, that is, the cube with the terminal vertex removed. Define $P_{n}$ via the pushout in $\mathcal{D}$

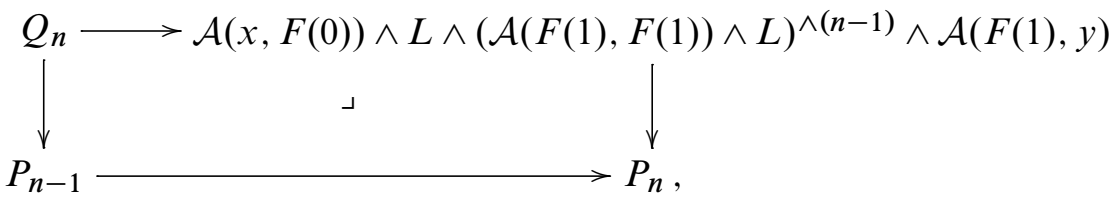

where the left vertical map is defined as follows: for each proper subset $S$ of $\{1,2, \ldots, n\}$ we consider the composed map

$$
W(S) \longrightarrow \underbrace{\mathcal{A}(x, F(0)) \wedge L \wedge \mathcal{A}(F(1), F(1)) \wedge \ldots \wedge L \wedge \mathcal{A}(F(1), y)}_{|S| \text { factors } L}
$$

obtained by first mapping each factor of $W(S)$ equal to $K$ to $\mathcal{A}(F(1), F(1))$, and then composing in $\mathcal{A}$ the adjacent factors. Finally, since $S$ is a proper subset, the right hand side belongs to $P_{|S|}$ and so to $P_{n+1}$. Now the same (conceptual) arguments as those of Schwede and Shipley [29, Lemma 6.2] show us that the above construction furnishes us a description of the $\mathcal{D}$-category $\mathcal{B}$.

We now analyse the constructed filtration. The cube $W$ used in the inductive definition of $P_{n}$ has $n+1$ factors of objects in $\mathcal{D}$, which map by the identity everywhere. Using the symmetry isomorphism of $-\wedge-$, we can shuffle them all to one side and observe that the map

$$
Q_{n} \longrightarrow \mathcal{A}(x, F(0)) \wedge L \wedge(\mathcal{A}(F(1), F(1)) \wedge L)^{\wedge(n-1)} \wedge \mathcal{A}(F(1), y)
$$

is isomorphic to

$$
\bar{Q}_{n} \wedge \mathcal{Z}_{n} \longrightarrow L^{\wedge n} \wedge \mathcal{Z}_{n}
$$


where

$$
\mathcal{Z}_{n}:=\mathcal{A}(x, F(0)) \wedge L \wedge(\mathcal{A}(F(1), F(1)) \wedge L)^{\wedge(n-1)} \wedge \mathcal{A}(F(1), y)
$$

and $\bar{Q}_{n}$ is the colimit of a punctured cube analogous to $W$, but with all the smash factors different from $K$ or $L$ deleted. By iterated application of the pushout product axiom, the map $\bar{Q}_{n} \rightarrow L^{\wedge n}$ is a trivial cofibration and so by the monoid axiom, the map $P_{n+1} \rightarrow P_{n}$ is a weak equivalence in $\mathcal{D}$. Since the map

$$
R(x, y): \mathcal{A}(x, y)=P_{0} \longrightarrow \mathcal{B}(x, y)
$$

is the kind of map considered in the monoid axiom, it is also a weak equivalence and so the Proposition is proven.

Proposition C.4 Let $\mathcal{A}$ be a $\mathcal{D}$-category such that $\mathcal{A}(x, y)$ is cofibrant in $\mathcal{D}$ for all $x, y \in \mathcal{A}$ and $i: N \rightarrow M$ a cofibration in $\mathcal{D}$. Then, in a pushout in $\mathcal{D}-C a t$

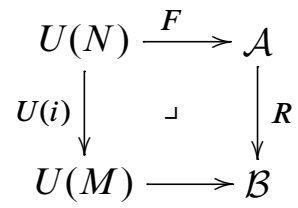

the morphisms

$$
R(x, y): \mathcal{A}(x, y) \longrightarrow \mathcal{B}(x, y), \quad x, y \in \mathcal{A}
$$

are all cofibrations in $\mathcal{D}$.

Proof The description of the morphisms

$$
R(x, y): \mathcal{A}(x, y) \longrightarrow \mathcal{B}(x, y), \quad x, y \in \mathcal{A}
$$

is analogous to the one of Proposition C.3. Since for all $x, y \in \mathcal{A}, \mathcal{A}(x, y)$ is cofibrant in $\mathcal{D}$, the pushout product axiom implies that in this situation the map

$$
\bar{Q}_{n} \wedge \mathcal{Z}_{n} \longrightarrow L^{\wedge n} \wedge \mathcal{Z}_{n}
$$

is a cofibration. Since cofibrations are stable under co-base change and transfinite composition, we conclude that the morphisms $R(x, y), x, y \in \mathcal{A}$ are cofibrations.

\section{Appendix D Proofs of Section 5}

\section{D.1 Proof of Theorem 5.5}

Let us denote by $\mathcal{W}_{L}$ the class of levelwise quasi-equivalences on $R-$ Cat. We start by observing that the category $R-$ Cat is complete and cocomplete. Since the restriction 
functor

$$
i^{*}: R-\mathrm{Cat} \longrightarrow \mathrm{Sp}^{\Sigma}-\mathrm{Cat}
$$

preserves filtered colimits and the domains and codomains of the elements of the generating (trivial) cofibrations in $\mathrm{Sp}^{\Sigma}$-Cat are small, condition (1) of the lifting Theorem (see Hirschhorn [20, Theorem 11.3.2]) is verified.

We now prove condition (2). Recall from Tabuada [37, Definition 4.5] the construction of the set $J$ of generating trivial cofibrations in $\mathrm{Sp}^{\Sigma}-\mathrm{Cat}$. We have

- a set $J^{\prime}$ of spectral functors

$$
A_{m, k, n}: U\left(F_{m} \Lambda[k, n]_{+}\right) \longrightarrow U\left(F_{m} \Delta[n]_{+}\right), m \geq 0, n \geq 1,0 \leq k \leq n .
$$

- a set $J^{\prime \prime}$ of spectral functors

$$
A_{\mathcal{H}}: \underline{\mathbb{S}} \longrightarrow \Sigma^{\infty}\left(\mathcal{H}_{+}\right) .
$$

Lemma D.1 $i_{!}\left(J^{\prime}\right)-$ cell $\subseteq \mathcal{W}_{L}$.

Proof Since the class $\mathcal{W}_{L}$ is stable under transfinite compositions it is enough to prove the following: let

$$
S: i_{!} U\left(F_{m} \Lambda[k, n]_{+}\right) \longrightarrow \mathcal{A}
$$

be a $R$-functor and consider the following pushout in $R-$ Cat

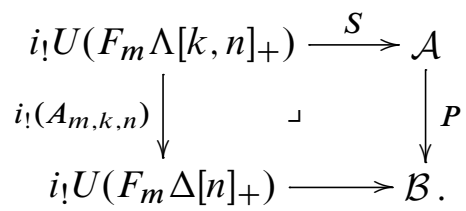

We need to show that $P$ belongs to $\mathcal{W}_{L}$. Observe that the $R$-functor $i_{!}\left(A_{m, k, n}\right)$ identifies with the $R$-functor

$$
U\left(i_{!}\left(F_{m} \Lambda[k, n]_{+}\right)\right) \longrightarrow U\left(i_{!}\left(F_{m} \Delta[n]_{+}\right)\right) .
$$

Since the morphisms

$$
i_{!}\left(F_{m} \Lambda[k, n]_{+}\right) \longrightarrow i_{!}\left(F_{m} \Delta[n]_{+}\right)
$$

are trivial cofibrations in the projective level model structure of Theorem 5.1, Proposition C. 3 and Theorem 5.1 imply that the spectral functor $i^{*}(P)$ satisfies condition (L1). Since $P$ induces the identity map on objects, the spectral functor $i^{*}(P)$ satisfies also condition (L2') which implies that $P$ belongs to $\mathcal{W}_{L}$.

Lemma D.2 $i_{!}\left(J^{\prime \prime}\right)-c e l l \subseteq \mathcal{W}_{L}$. 
Proof Since the class $\mathcal{W}_{L}$ is stable under transfinite compositions, it is enough to prove the following: let

$$
S: i ! \underline{\mathbb{S}}=\underline{R} \longrightarrow \mathcal{A}
$$

be a $R$-functor (where $\underline{R}$ is the category with one object and endomorphism ring $R$ ) and consider the following pushout in $R$-cat

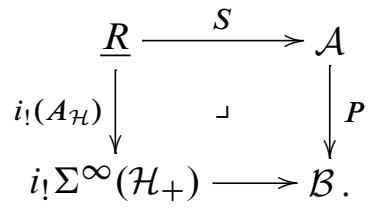

We need to show that $P$ belongs to $\mathcal{W}_{L}$. The proof concerning condition (L1) is entirely analogous to the proof of condition (L1) in Proposition 3.25. We now show that the spectral functor $i^{*}(P)$ satisfies condition (L2'). Notice that the existence of the spectral functor

$$
\Sigma^{\infty}\left(\mathcal{H}_{+}\right) \longrightarrow i^{*} i_{!} \Sigma^{\infty}\left(\mathcal{H}_{+}\right),
$$

given by the unit of the adjunction $\left(i_{!}, i^{*}\right)$, implies that the left vertical spectral functor in the following commutative square

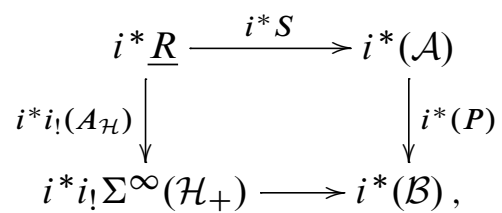

satisfies condition (L2'). Notice also, that if we restrict ourselves to the objects of each category $(\operatorname{obj}(-))$ in the previous diagram, we obtain a co-cartesian square

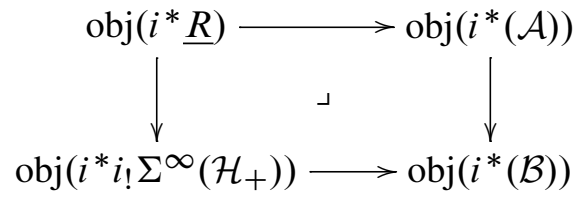

in Set. These facts shows us that $i^{*}(P)$ satisfies condition $\left(\mathrm{L} 2^{\prime}\right)$ and so we conclude that $P$ belongs to $\mathcal{W}_{L}$.

Lemmas D.1 and D.2 imply condition (2) of Hirschhorn [20, Theorem 11.3.2] and so the proof of Theorem 5.5 is finished. 


\section{D.2 Proof of Proposition 5.7}

The model structure of Theorem 5.5 is cofibrantly generated and so every cofibrant object in $R$-Cat is a retract of a $I$-cell complex, see Hirschhorn [20, Corollary 11.2.2]. Since cofibrations are stable under filtered colimits, it is enough to prove the Proposition for pushouts along a generating cofibration. Recall from Tabuada [37, Definition 4.4] the construction of the set $I$ of generating cofibrations in $\mathrm{Sp}^{\Sigma}-\mathrm{Cat}$. We have

- a spectral functor

$$
C: \varnothing \longrightarrow \underline{\mathbb{S}} .
$$

- spectral functors

$$
C_{m, n}: U\left(F_{m} \partial \Delta[n]_{+}\right) \longrightarrow U\left(F_{m} \Delta[n]_{+}\right), m, n \geq 0 .
$$

Let $\mathcal{A}$ be a $R$-category such that $\mathcal{A}(x, y)$ is cofibrant for all objects $x, y \in \mathcal{A}$ :

- Consider the following pushout

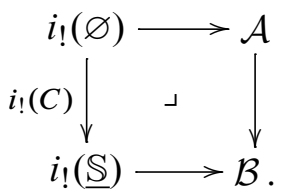

Notice that $\mathcal{B}$ is obtained simply from $\mathcal{A}$ by introducing a new object. It is then clear that for all objects $x, y \in \mathcal{B}$, the $R$-module $\mathcal{B}(x, y)$ is cofibrant in the projective level model structure on $R$-Mod.

- Now, consider the following pushout

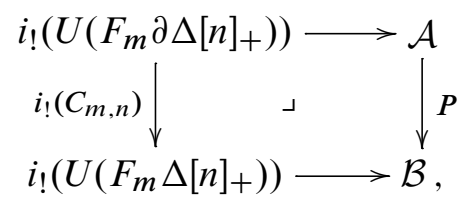

Notice that $\mathcal{A}$ and $\mathcal{B}$ have the same set of objects and that $P$ induces the identity map on the object sets. Moreover the $R$-functor $i_{!}\left(C_{m, n}\right)$ identifies with the $R$-functor

$$
U\left(i_{!}\left(F_{m} \partial \Delta[n]_{+}\right)\right) \longrightarrow U\left(i_{!}\left(F_{m} \Delta[n]_{+}\right)\right) .
$$

Since the morphisms

$$
i_{!}\left(F_{m} \partial \Delta[n]_{+}\right) \longrightarrow i_{!}\left(F_{m} \Delta[n]_{+}\right)
$$


are cofibrations in the projective level model structure of Theorem 5.1, Proposition C.4 and Theorem 5.1 imply that the morphism

$$
P(x, y): \mathcal{A}(x, y) \longrightarrow \mathcal{B}(x, y)
$$

is still a cofibration. Since the $I-$ cell complexes in $R-$ Cat are built of $\varnothing$ (the initial object), the Proposition is proven.

Lemma D.3 The functor

$$
U: R-\operatorname{Mod} \longrightarrow R-C a t \quad \text { (see Definition C.1) }
$$

sends projective cofibrations to cofibrations.

Proof The model structure of Theorem 5.5 is cofibrantly generated and so any cofibrant object in $R$-Cat is a retract of a (possibly infinite) composition of pushouts along the generating cofibrations. Since the functor $U$ preserves retractions, colimits and send the generating projective cofibrations to (generating) cofibrations the Lemma is proven.

\section{D.3 Proof of Proposition 5.11}

The definition of stable equivalence in $R$-Mod (see Theorem 5.1), implies that a $R-$ functor $F$ satisfies condition (WE1) if and only if the spectral functor $i^{*}(F)$ satisfies condition (S1). Now, let $\mathcal{A}$ be a $R$-category. Observe that, since the functor

$$
i ! \mathrm{Sp}^{\Sigma} \longrightarrow R-\mathrm{Mod}
$$

is strong monoidal, the category $[\mathcal{A}]$ (see Remark 2.7 ) naturally identifies with $\left[i^{*}(\mathcal{A})\right]$ which implies that $F$ satisfies condition (WE2) if and only if $i^{*}(F)$ satisfies condition (S2). This finishes the proof.

\section{D.4 Proof of Proposition 5.14}

Since the elements of the set $\left\{A_{m, n, k}\right\} \cup K$ are trivial cofibrations in the projective stable model structure on $\mathrm{Sp}^{\Sigma}$, the elements of the set $\left\{i_{!}\left(A_{m, n, k}\right)\right\} \cup i_{!}(K)$ are trivial cofibrations in the stable model structure on $R$-Mod (see Theorem 5.1). By Theorem 5.1 this model structure is monoidal and satisfies the monoid axiom. This implies, by Proposition C.3, that the spectral functor $\eta_{\mathcal{A}}$ satisfies condition (WE1) (see Definition 2.8). Since $Q(\mathcal{A})$ and $\mathcal{A}$ have the same object sets, condition (WE2) is automatically verified and so we conclude that $\eta_{\mathcal{A}}$ belongs to $\mathcal{W}_{S}$. By Remark 5.9, the proof is finished. 


\section{D.5 Proof of Lemma 5.16}

We have at our disposal a commutative square

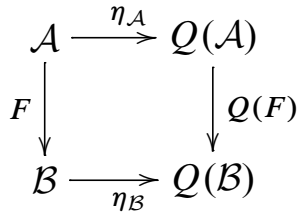

where the $R$-functors $\eta_{\mathcal{A}}$ and $\eta_{\mathcal{B}}$ are stable quasi-equivalences by Proposition 5.14. If we apply the restriction functor $i^{*}$ to the above square, we obtain

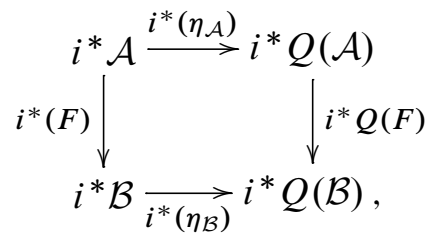

where the spectral functors $i^{*}\left(\eta_{\mathcal{A}}\right)$ and $i^{*}\left(\eta_{\mathcal{B}}\right)$ are stable quasi-equivalences in $\mathrm{Sp}{ }^{\Sigma}$-Cat (see Proposition 5.11) and the spectral categories $i^{*} Q(\mathcal{A})$ and $i^{*} Q(\mathcal{B})$ satisfy condition $(\Omega)$ (see Remark 5.13). Now, we conclude: $F$ is a stable weak equivalence if and only if $i^{*}(F)$ is a stable quasi-equivalence; if and only if $i^{*} Q(F)$ is a stable quasiequivalence; if and only if $i^{*} Q(F)$ is a levelwise quasi-equivalence (see Proposition $3.44)$; if and only if $F$ is a $Q$-weak equivalence.

\section{D.6 Proof of Theorem 5.17}

The proof will consist on verifying the conditions of Theorem B.2. We consider for $\mathcal{M}$ the Quillen model structure of Theorem 5.5 and for $Q$ and $\eta$, the functor and natural transformation of Definition 5.12. The Quillen model structure of Theorem 5.5 is right proper (see Proposition 3.29) and by Lemma 5.16, the $Q$-weak equivalences are precisely the stable quasi-equivalences. We now verify conditions (A1), (A2) and (A3):

(A1) Let $F: \mathcal{A} \rightarrow \mathcal{B}$ be a levelwise quasi-equivalence. We have the following commutative square

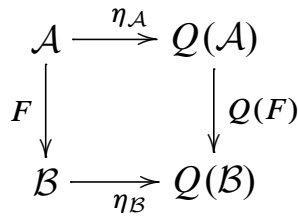


with $\eta_{\mathcal{A}}$ and $\eta_{\mathcal{B}}$ stable quasi-equivalences. If we apply the restriction functor $i^{*}$ to the above square, we obtain

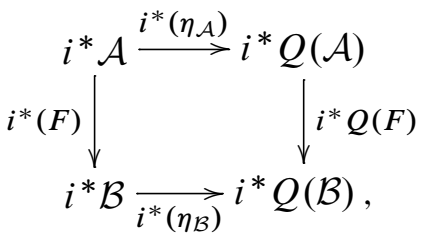

with $i^{*}(F)$ a levelwise quasi-equivalence, $i^{*}\left(\eta_{\mathcal{A}}\right)$ and $i^{*}\left(\eta_{\mathcal{B}}\right)$ stable quasiequivalences and $i^{*} Q(\mathcal{A})$ and $i^{*} Q(\mathcal{B})$ satisfying the condition $(\Omega)$ of Remark 5.13. Now, condition (A1) of Theorem 3.46 implies condition (A1).

(A2) We now show that for every $R$-category $\mathcal{A}$, the $R$-functors

$$
\eta_{Q(\mathcal{A})}, Q\left(\eta_{\mathcal{A}}\right): Q(\mathcal{A}) \longrightarrow Q Q(\mathcal{A})
$$

are stable quasi-equivalences. If we apply the restriction functor $i^{*}$ to the above $R$-functors, we obtain stable quasi-equivalences between spectral categories which satisfy condition $(\Omega)$. Now, condition (A2) of Theorem 3.46 implies condition (A2).

(A3) We start by observing that if $P: \mathcal{A} \longrightarrow \mathcal{D}$ is a $Q$-fibration, then for all objects $x, y \in \mathcal{C}$, the morphism

$$
P(x, y): \mathcal{C}(x, y) \longrightarrow \mathcal{D}(P x, P y)
$$

is a fibration in the projective stable model structure on $R-$ Mod (see Theorem 5.1). In fact, by Lemma D.3, the functor

$$
U: R-\operatorname{Mod} \longrightarrow R-\text { Cat }
$$

sends projective cofibrations to cofibrations. Since it sends stable equivalences to stable quasi-equivalences the claim follows. Now consider the following diagram in $R-$ Cat

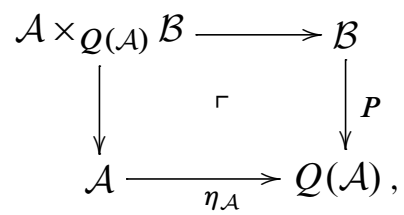


with $P$ a $Q$-fibration. If we apply the restriction functor $i^{*}$ to the previous commutative square, we obtain a cartesian square

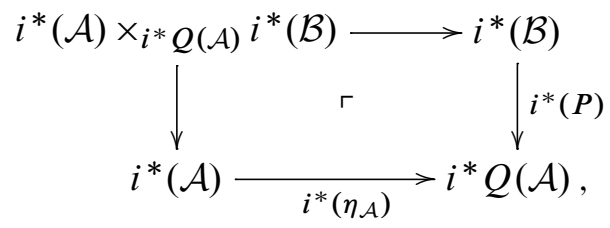

with $i^{*}\left(\eta_{\mathcal{A}}\right)$ a stable quasi-equivalence. Notice also that $i^{*}(P)$ is such that for all objects $x, y \in i^{*}(\mathcal{B})$, the morphism

$$
i^{*}(P)(x, y): i^{*}(\mathcal{B})(x, y) \longrightarrow i^{*} Q(\mathcal{A})
$$

is a stable fibration in $\mathrm{Sp}^{\Sigma}$. Now, condition (A3) of Theorem 3.46 implies condition (A3).

The Theorem is now proved.

\section{D.7 Proof of Proposition 5.18}

By Corollary B.4, $\mathcal{A}$ is fibrant, with respect to Theorem 5.17 if and only if it is fibrant for the structure of Theorem 5.5 (see Remark 5.6) and the $R$-functor $\eta_{\mathcal{A}}: \mathcal{A} \rightarrow Q(\mathcal{A})$ is a levelwise quasi-equivalence. Observe that

$$
i^{*}\left(\eta_{\mathcal{A}}\right): i^{*}(\mathcal{A}) \rightarrow i^{*} Q(\mathcal{A})
$$

is a levelwise quasi-equivalence if and only if for all objects $x, y \in \mathcal{A}$, the morphism of symmetric spectra

$$
i^{*}\left(\eta_{\mathcal{A}}(x, y)\right): i^{*} \mathcal{A}(x, y) \longrightarrow i^{*} Q(\mathcal{A})(x, y)
$$

is a level equivalence. Since for all objects $x, y \in \mathcal{A}$, the symmetric spectrum $i^{*} Q(\mathcal{A})(x, y)$ is an $\Omega$-spectrum (see Remark 5.13) we have the following commutative diagrams (for all $n \geq 0$ )

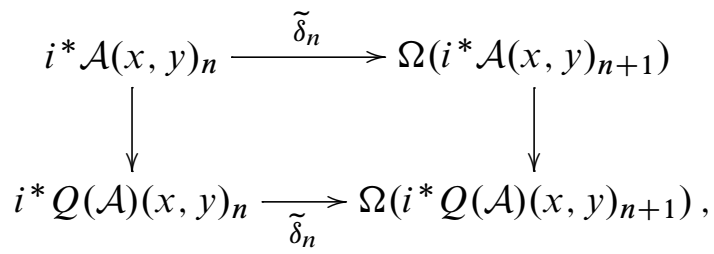


where the bottom and vertical arrows are weak equivalences of pointed simplicial sets. This implies that

$$
\tilde{\delta}_{n}: i^{*} \mathcal{A}(x, y)_{n} \longrightarrow \Omega\left(i^{*} \mathcal{A}(x, y)_{n+1}\right), n \geq 0
$$

is a weak equivalence of pointed simplicial sets. In conclusion, for all objects $x, y \in \mathcal{A}$, the symmetric spectrum $i^{*} \mathcal{A}(x, y)$ is an $\Omega$-spectrum.

\section{D.8 Proof of Proposition 5.21}

We start with the model structure of Theorem 5.5. Notice that the commutative diagram

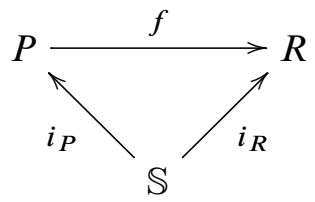

induces a strictly commutative diagram of categories

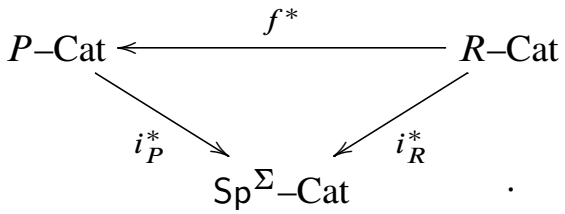

By Theorem 5.5, we conclude that $f^{*}$ preserves (and reflects) levelwise quasi-equivalences and fibrations. To show the Proposition with respect to the model structure of Theorem 5.17, we verify conditions (a) and (b) of Proposition 2.13.

(a) Clearly $f^{*}$ preserves trivial fibrations, since these are the same in the Quillen model structures of Theorems 5.5 and 5.17.

(b) Let $F: \mathcal{A} \rightarrow \mathcal{B}$ be a $Q$-fibration in $R$-Cat between fibrant objects (see Proposition 5.18). By Theorem B.3, $F$ is a fibration and so by condition (a) so is $f^{*}(F)$. Proposition 5.18 and the above strictly commutative diagram of categories imply that $f^{*}$ preserves $Q$-fibrant objects. By the following Lemma D.4, we conclude that $f^{*}(F)$ is a $Q$-fibration.

Lemma D.4 Let $F: \mathcal{A} \rightarrow \mathcal{B}$ be a fibration (in the model structure of Theorem 5.5) between $Q$-fibrant objects (see Proposition 5.18). Then $F$ is a $Q$-fibration. 
Proof We need to show (by Theorem B.3) that the following commutative square

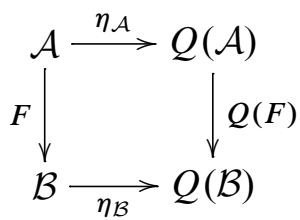

is homotopy cartesian (in the model structure of Theorem 5.5). By Theorem 5.5, this is equivalent to the fact that the following commutative square in $\mathrm{Sp}^{{ }^{\Sigma}}$-Cat

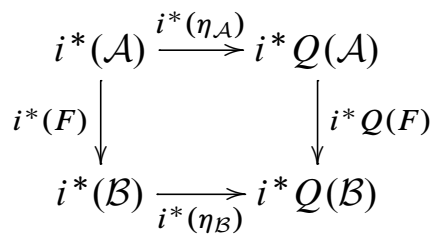

is homotopy cartesian. Notice that since $\mathcal{A}$ and $\mathcal{B}$ are $Q$-fibrant objects, the spectral functors $i^{*}\left(\eta_{\mathcal{A}}\right)$ and $i^{*}\left(\eta_{\mathcal{B}}\right)$ are levelwise quasi-equivalences. Since $i^{*}(F)$ is a fibration, the Proposition is proven.

\section{D.9 Proof of Proposition 5.22}

In order to simplify the notation, we will denote by $Q$ the $Q$-functor on $R$-Cat and on $\mathrm{Sp}^{\Sigma}$-Cat. In each situation it will be clear from the context which functor we refer to. Proposition 5.21 (with $P=\mathbb{S}$ ) shows us that if $F$ is a $Q$-fibration then $i^{*}(Q)$ is $Q$-fibration in $\mathrm{Sp}^{\Sigma}$-Cat. Let us now prove the converse. If $i^{*}(F)$ is a $Q$-fibration in $\mathrm{Sp}^{\Sigma}$-Cat, Theorem B.3 implies that $i^{*}(F)$ is a fibration and that we have a homotopy cartesian square in $\mathrm{Sp}^{\Sigma}$-Cat

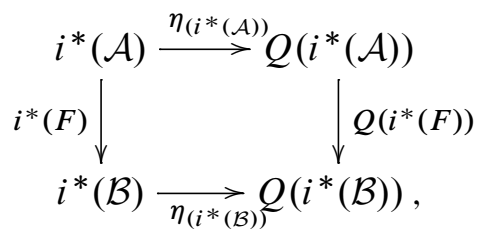

with respect to the model structure of Theorem 3.19. Now, consider the following square in $R-$ Cat

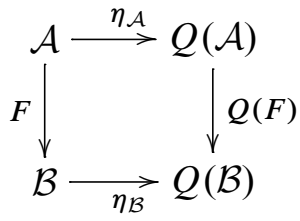


and apply to it the restriction of scalars functor $i^{*}(-)$. Since the functor $Q$ in $\mathrm{Sp}^{\Sigma}-\mathrm{Cat}$ is constructed by the small object argument, and since $i^{*} Q(\mathcal{A})$ and $i^{*} Q(\mathcal{B})$ satisfy condition $(\Omega)$ of Remark 5.13, we can construct (by an inductive procedure) spectral functors $\psi_{\mathcal{A}}$ and $\psi_{\mathcal{B}}$ making the following diagram commute

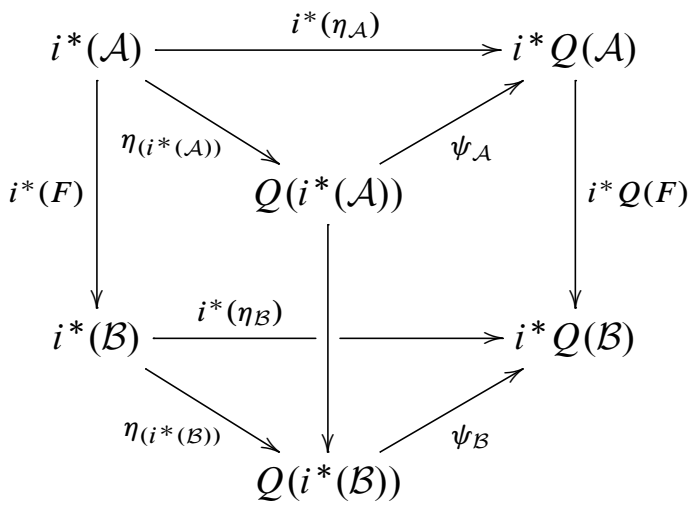

Since $\eta_{\left(i^{*}(\mathcal{A})\right)}, \eta_{\left(i^{*}(\mathcal{B})\right)}, i^{*}\left(\eta_{\mathcal{A}}\right)$ and $i^{*}\left(\eta_{\mathcal{B}}\right)$ are all stable quasi-equivalences, the twoout-of-three property implies that $\psi_{\mathcal{A}}$ and $\psi_{\mathcal{B}}$ are also stable quasi-equivalences. Moreover, since the spectral categories $i^{*} Q(\mathcal{A}), i^{*} Q(\mathcal{B}), Q\left(i^{*}(\mathcal{A})\right)$ and $Q\left(i^{*}(\mathcal{B})\right)$ are $Q$-fibrant, Proposition 3.44 implies that $\psi_{\mathcal{A}}$ and $\psi_{\mathcal{B}}$ are levelwise quasi-equivalences. This implies that the square

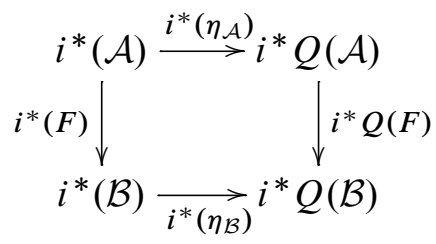

is also homotopy cartesian. Finally, since the restriction of scalars functor $i^{*}(-)$ preserves limits and preserves and reflects levelwise quasi-equivalences and fibrations (see Definition 5.4), we conclude that the square in $R-$ Cat

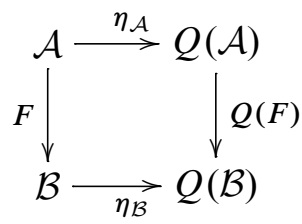

is homotopy cartesian with $F$ a fibration. By Theorem B.3, this implies that $F$ is a $Q$-fibration in $R-$ Cat and so the proof is finished. 


\section{References}

[1] J E Bergner, A model category structure on the category of simplicial categories, Trans. Amer. Math. Soc. 359 (2007) 2043-2058 MR2276611

[2] A Blumberg, M Mandell, Localization theorems in topological Hochschild homology and topological cyclic homology arXiv:0802.3938

[3] M Bökstedt, Topological Hochschild homology, preprint (1990)

[4] F Borceux, Handbook of categorical algebra. 2, Encyclopedia of Mathematics and its Applications 51, Cambridge University Press, Cambridge (1994) MR1313497Categories and structures

[5] D-C Cisinski, A Neeman, Additivity for derivator K-theory, Adv. Math. 217 (2008) 1381-1475 MR2382732

[6] V Drinfeld, DG quotients of DG categories, J. Algebra 272 (2004) 643-691 MR2028075

[7] D Dugger, Replacing model categories with simplicial ones, Trans. Amer. Math. Soc. 353 (2001) 5003-5027 MR1852091

[8] D Dugger, Spectral enrichments of model categories, Homology, Homotopy Appl. 8 (2006) 1-30 MR2205213

[9] D Dugger, B Shipley, Topological equivalences for differential graded algebras, Adv. Math. 212 (2007) 37-61 MR2319762

[10] B I Dundas, T Goodwillie, R McCarthy, The local structure of algebraic $K$-theory Available at http://www.math.ntnu.no/ dundas/indexeng.html

[11] B I Dundas, R McCarthy, Topological Hochschild homology of ring functors and exact categories, J. Pure Appl. Algebra 109 (1996) 231-294 MR1388700

[12] W G Dwyer, D M Kan, Function complexes in homotopical algebra, Topology 19 (1980) 427-440 MR584566

[13] W G Dwyer, D M Kan, Simplicial localizations of categories, J. Pure Appl. Algebra 17 (1980) 267-284 MR579087

[14] T Geisser, L Hesselholt, Topological cyclic homology of schemes, from: "Algebraic $K$-theory (Seattle, WA, 1997)”, Proc. Sympos. Pure Math. 67, Amer. Math. Soc., Providence, RI (1999) 41-87 MR1743237

[15] P G Goerss, J F Jardine, Simplicial homotopy theory, Progress in Mathematics 174, Birkhäuser Verlag, Basel (1999) MR1711612

[16] A Grothendieck, Les Dérivateurs Available at http:// people.math.jussieu.fr/ maltsin/groth/Derivateurs.html

[17] L Hesselholt, I Madsen, Cyclic polytopes and the $K$-theory of truncated polynomial algebras, Invent. Math. 130 (1997) 73-97 MR1471886 
[18] L Hesselholt, I Madsen, On the $K$-theory of finite algebras over Witt vectors of perfect fields, Topology 36 (1997) 29-101 MR1410465

[19] L Hesselholt, I Madsen, On the K-theory of local fields, Ann. of Math. (2) 158 (2003) 1-113 MR1998478

[20] PS Hirschhorn, Model categories and their localizations, Mathematical Surveys and Monographs 99, American Mathematical Society, Providence, RI (2003) MR1944041

[21] M Hovey, Model categories, Mathematical Surveys and Monographs 63, American Mathematical Society, Providence, RI (1999) MR1650134

[22] M Hovey, Spectra and symmetric spectra in general model categories, J. Pure Appl. Algebra 165 (2001) 63-127 MR1860878

[23] M Hovey, B Shipley, J Smith, Symmetric spectra, J. Amer. Math. Soc. 13 (2000) 149-208 MR1695653

[24] A Joyal, M Tierney, Strong stacks and classifying spaces, from: "Category theory (Como, 1990)", Lecture Notes in Math. 1488, Springer, Berlin (1991) 213-236 MR1173014

[25] B Keller, On differential graded categories, from: "International Congress of Mathematicians Vol II”, Eur. Math. Soc., Zürich (2006) 151-190 MR2275593

[26] S Mac Lane, Categories for the working mathematician, second edition, Graduate Texts in Mathematics 5, Springer, New York (1998) MR1712872

[27] D G Quillen, Homotopical algebra, Lecture Notes in Mathematics 43, Springer, Berlin (1967) MR0223432

[28] S Schwede, An untitled book project about symmetric spectra Available at http:// www.math.uni-bonn.de/people/schwede/

[29] S Schwede, B E Shipley, Algebras and modules in monoidal model categories, Proc. London Math. Soc. (3) 80 (2000) 491-511 MR1734325

[30] S Schwede, B Shipley, Equivalences of monoidal model categories, Algebr. Geom. Topol. 3 (2003) 287-334 MR1997322

[31] S Schwede, B Shipley, Stable model categories are categories of modules, Topology 42 (2003) 103-153 MR1928647

[32] B Shipley, $H \mathbb{Z}$-algebra spectra are differential graded algebras, Amer. J. Math. 129 (2007) 351-379 MR2306038

[33] A E Stanculescu, A model category structure on the category of simplicial multicategories arXiv:0805.2611

[34] G Tabuada, Une structure de catégorie de modèles de Quillen sur la catégorie des dg-catégories, C. R. Math. Acad. Sci. Paris 340 (2005) 15-19 MR2112034

[35] G Tabuada, Théorie homotopique des DG-catégories, doctoral thesis, Université Paris VII (2007) arXiv:0710.4303 
[36] G Tabuada, Higher K-theory via universal invariants, Duke Math. J. 145 (2008) 121-206 MR2451292

[37] G Tabuada, Homotopy theory of spectral categories, Adv. Math. 221 (2009) 1122-1143 MR2518634

[38] G Tabuada, Differential graded versus Simplicial categories, Topol. Appl. 157 (2010) 563-593

[39] R W Thomason, Algebraic K-theory and étale cohomology, Ann. Sci. École Norm. Sup. (4) 18 (1985) 437-552 MR826102

[40] F Waldhausen, Algebraic K-theory of spaces, from: "Algebraic and geometric topology (New Brunswick, N.J., 1983)", Lecture Notes in Math. 1126, Springer, Berlin (1985) 318-419 MR802796

Departamento de Matemática e CMA, FCT-UNL, Quinta da Torre 2829-516 Caparica, Portugal

tabuada@fct.unl.pt

Received: 18 September 2008 Revised: 28 July 2009 